\title{
8 Comets: Potential Sources of Prebiotic Molecules for the Early Earth
}

\author{
Didier Despois, Hervé Cottin
}

\section{"Of Ice and Men..."}

Why should we be interested in comets when studying the origin of life on Earth? First, comets are rich in water and carbon, two essential constituents of terrestrial life; part of Earth's water and carbon might be of cometary origin. But comets might also have brought Earth one step further on the way to the emergence of life.

Early last century, Chamberlin and Chamberlin proposed that infalling carbonaceous chondrite meteorites could have been an important source of terrestrial organic compounds (Chamberlin and Chamberlin 1908). Oró was the first in 1961 to propose from observations of carbon- and nitrogen-containing radicals in cometary comae that comets may have played a similar role (Oró 1961):

"I suggest that one of the important consequences of the interactions of comets with the Earth would be the accumulation on our planet of relatively large amounts of carbon compounds which are known to be transformed spontaneously into amino acids, purines and other biochemical compounds."

It is now quite obvious, from observations and laboratory experiments, that comets are important reservoirs of a wide variety of organic compounds.

Comets will be considered in this chapter mainly as potential reservoirs of carbon and organic molecules for the early Earth. We present first their general characteristics, then the chemical composition of cometary matter as deduced from observations and constrained by laboratory experiments, the origin and evolution of this matter - and of comets themselves - the various cometary contributions to the early Earth, and the main future of ground-based and space developments for comet science. We conclude with a list of important questions for which answers should and will be sought in the forthcoming years.

The book "Comets and the Origin and Evolution of Life", edited by Thomas et al. (1997), is a basic reference on the topic. Comets have been discussed as possible sources of prebiotic molecules by Delsemme (2000), Greenberg (e.g. 1982, 1993, 1998) and Chyba and Sagan (1997). Despois et al. (2002) and Crovisier (2004) have discussed recently the observed molecular content of comets in this perspective, while Whittet (1997) and Ehrenfreund et al. (2002) relate more generally astrochemistry to the origin of Life. Recent general reviews on cometary 
science are Irvine et al. (2000), Festou et al. (1993a,b), and Bockelée-Morvan et al. (2004); the book Comets II (in press) edited by Festou, Keller and Weaver will present up-to-date information on many aspects of comets. In this book, Petit and Morbidelli (2004) survey the Solar System formation, and discuss the origin of water on Earth. Chapters of the French edition of this book (Gargaud et al., 2001, 2003), not included in this English edition, also contain much information on related topics only briefly alluded to here: build up and evolution of the Earth atmosphere (Marty; Selsis and Parisot), build up of the hydrosphere and $\mathrm{D} / \mathrm{H}$ ratio (Robert), micrometeorites (Maurette). Recent related papers in English are Kasting (1993, 2003), Dauphas et al. (2000), Robert (2002), and Maurette $(1998,2000)$.

\subsection{General Description of Comets}

"Comets are small bodies of the Solar System, made of a mixture of ices and dust."

\subsubsection{The Cometary Nucleus}

The definition above describes in fact a specific part of comets, the cometary nucleus. Comets are most of the time far from the Sun and reduced to this nucleus, a solid body named a "dirty ice snow-ball" by Whipple (1950); it is only when comets get close enough to the Sun (less than a few UA) that they develop their other more spectacular and better known components - coma and tail - described below. Comet $1 \mathrm{P} / \mathrm{Halley}^{1}$ is the first of the only three comets whose nuclei have been closely observed by a space probe (the other two are 19P/Borrelly, observed from $3400 \mathrm{~km}$ by the Deep Space 1 spacecraft in September 2001, and P/Wild 2 observed by the Stardust spacecraft from $236 \mathrm{~km}$ in January 2004). In 1986, several probes were sent to observe comet Halley, among which the European Space Agency probe "Giotto" passed within approximately $500 \mathrm{~km}$ from the comet nucleus. The pictures taken by Giotto (Fig. 8.1) revealed an irregular-shaped body $8 \times 8 \times 16 \mathrm{~km}$ by size, whose volume is roughly equivalent to a sphere of $10 \mathrm{~km}$ in diameter (see Newburn et al., 1991). The bright comet C/1996 B2 Hyakutake, which appeared in 1996 and passed rather close to the Earth $(\sim 0.1 \mathrm{AU})$, had a relatively small nucleus, about $3 \mathrm{~km}$ in diameter. Another comet, C1995 O1/Hale-Bopp, was detected for the first time during summer 1995 and became quite visible in spring 1997; although passing ten times further from the Earth, it has been more spectacular than Hyakutake due to its much larger diameter, approximately $45 \mathrm{~km}$ (see Weaver and Lamy 1999).

\footnotetext{
${ }^{1} 1 \mathrm{P} /$ Halley is the official complete name of the comet, according to the International Astronomical Union - see ref. CBAT for a description of the naming rules; the familiar names (e.g. "comet Halley") are also used in this chapter and should be considered as abbreviations.
} 

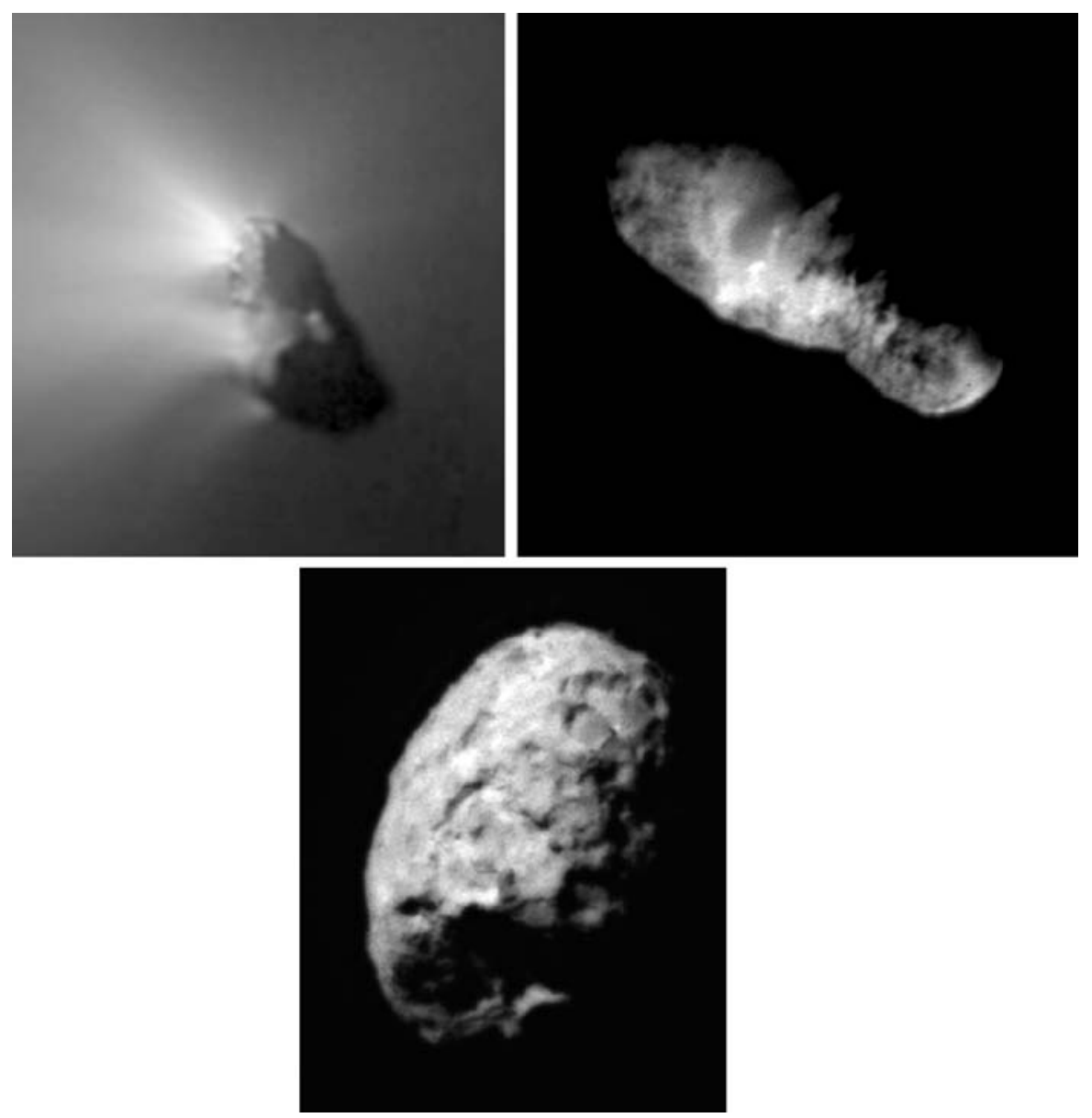

Fig. 8.1. (a) The nucleus of comet Halley, as seen by the ESA probe Giotto in 1986. (c) ESA and MPIfA) (b) the nucleus of comet P/Borrelly observed by NASA probe Deep Space 1 in 2001 (c) NASA) (c) the nucleus of comet P/Wild 2 recently observed (Jan. 2004) with a resolution of $20 \mathrm{~m}$ by Stardust, the NASA probe that will return dust samples collected during the flyby (C) NASA)

The density of cometary nuclei is difficult to measure precisely: some estimates are as low as $0.2 \mathrm{~g} / \mathrm{cm}^{3}$ or as high as $1.8 \mathrm{~g} / \mathrm{cm}^{3}$. However, most values are in the range $0.5-0.9 \mathrm{~g} / \mathrm{cm}^{3}$ (e.g. Ball et al., 2001). This is lower than the density of water ice, and would confirm the image of the "dirty snowball". Using this density, the mass of comet Halley is about $10^{18} \mathrm{~g}$ (1000 billion tonnes).

The cohesion of cometary nuclei is low at least in some cases, as shown by comet D/1993 F2 Shoemaker-Levy 9: when the comet passed near Jupiter, the tidal forces let the nucleus split up into a swarm of more than 20 major pieces, which later crashed onto the planet (see Noll et al., 1996). The fact 
that cometary nuclei have irregular shapes shows that these bodies were never molten - otherwise they would be shaped according to the equilibrium figure of a liquid body: a sphere or, if the body rotates, an ellipsoid.

What are the ices and dust mentioned in the definition of a comet made of? Cometary ices are water ice mostly, mixed with smaller quantities of other species like methanol $\left(\mathrm{CH}_{3} \mathrm{OH}\right), \mathrm{CO}, \mathrm{CO}_{2}, \ldots$ - the detailed composition is given below (Sect. 8.2.1 and Table 8.1). As for the dust grains, they are made of small particles of carbon, organic matter or silicates. The total mass of dust is roughly comparable to the mass of ices, within a factor of 10: some comets are "dusty", while other are "gaseous" (e.g. Rolfe and Battrick, 1987). Despite the presence of a large amount of ice inside the nucleus, the surface itself appears quite dark: for comet Halley, the proportion of reflected light (called albedo) is only $4 \%$.

\subsubsection{Comet Motion}

Comets move around the Sun following orbits that are in most cases ellipses (the Sun being at one focus); in some cases the orbit may be parabolic or slightly hyperbolic (e.g. Festou et al., 1993a). The eccentricity is generally high, and often near 1 (parabola) or even slightly higher (hyperbola). For comparison, the eccentricity of a circle is zero, and that of the quasicircular orbit of the Earth is 0.017 (at present). The comet speed in the region of the Earth's orbit (i.e. at one AU from the Sun) is about $45 \mathrm{~km} / \mathrm{s}$ (or $162000 \mathrm{~km} / \mathrm{h}$ ). Combining (vectorially) this speed with Earth's own motion around the Sun $(\sim 30 \mathrm{~km} / \mathrm{s})$ leads to relative speeds ranging between 15 and $75 \mathrm{~km} / \mathrm{s}$. This confers a large kinetic energy to cometary particles entering the Earth's atmosphere and leads to dramatic effects when the nucleus itself impacts the Earth.

Comets are classified into various families according to their orbits. Longperiod comets are defined as having an orbital period above 200 years. Among the long-period comets, one commonly calls "new comets" those for which aphelion (point of the trajectory furthest away from the Sun) is located beyond approximately $1000 \mathrm{AU}$ and for which eccentricity is close to 1 . This is not an official, well-defined, comet category; as these comets have periods above 10000 years (and up to tens of Myr), no historical record of previous passages exists, and they appear "new" to us. Their orbits are very elongated ellipses, which, when they pass close to the Sun, look very much like parabolas. Comets with periods below 200 years are called short-period comets. Among those, one finds the Halley family, with periods from 20 to 200 years, and random inclinations of their orbital plane with respect to the ecliptic, and the Jupiter family, with periods of approximately 6 years, and small inclinations to the ecliptic.

\subsubsection{Comet Reservoirs: Oort Cloud and Kuiper Belt}

To explain the present number of observed comets (despite the rapid sublimation of the ice (see Sect. 8.1.5) and the origin of the various orbital families, the existence of two comet reservoirs is required. 
The classical or outer Oort cloud (Oort, 1950) is a spherical reservoir situated between $\sim 20000$ and $100000 \mathrm{AU}$; the inner radius is loosely defined (see below), whereas the outer radius corresponds to half the distance to the nearest stars, beyond which an object can no longer be considered gravitationnally linked to the Sun. Oort Cloud comets are thought to be icy planetesimals scattered by the giant planets, originating mostly from the Uranus-Neptune zone and to a lesser extent from the Jupiter-Saturn zone.

Due to stellar encounters, or to the effect of the gravitational potential of the Galaxy, some of these objects are regularly sent back towards the planet zone with nearly parabolic orbits ("new" comets). The possible quantitative importance of a more tightly bound inner Oort cloud (3000-20000AU), has been recently underlined (Fernandez, 1997, Levison et al. 2001) considering the very probable option that the Solar System and its Oort cloud formed in the dense stellar environment of a young open star cluster. Formation scenarios of the Oort cloud (e.g. Duncan et al., 1987) are still very uncertain, at least quantitatively. The total number of comets in the Oort cloud could be on the order of $10^{12}-10^{14}$ depending on the model and the size distribution, while the total mass estimate ranges from 1 to $250 M_{\text {Earth }}$.

While the Oort cloud hypothesis explains quite well the long-period comet distribution, numerical simulations (Fernandez 1980) have stressed the importance of another reservoir, the Kuiper Belt, to explain the number of short-period comets. The existence of this belt was proposed first by Leonard (1930) and Edgeworth (1943), (the history of the concept, including the Kuiper (1950) paper and the later role of Cameron and Whipple, is discussed in Green 1999). The region has the shape of a thick torus and contains many small icy bodies beyond the orbit of Neptune. Since the discovery of the first Kuiper Belt object (KBO) by Jewitt and Luu in 1992, much observational and theoretical work has been undertaken (see Morbidelli and Brown (2004), Jewitt (2002) and de Bergh (2004) for recent reviews on the dynamical and physical properties of KBOs). Collisions in the Kuiper belt are thought to produce fragments, which are later scattered by Neptune and the other planets towards the inner Solar System, first as Centaurs, then as Jupiter Family comets. From the $\sim 800$ objects now observed (summer 2003) a total population of 70000 bodies larger than $100 \mathrm{~km}$ in diameter is estimated, and 1 to 10 billion bodies larger than $1 \mathrm{~km}$. Several families, with probably different origin, are recognized in the Kuiper Belt (see Morbidelli and Brown 2002 and Jewitt 2002 for details). A subpopulation, the plutinos, is in a collision-avoiding resonant orbit with Neptune, and encompasses Pluto itself (and its satellite Charon). Pluto is considered as the largest KBO (with $D \sim 2500 \mathrm{~km}$ ), and several other large objects are known (Quaoar, $D \sim 1300 \mathrm{~km}$; Charon, $D \sim 1200 \mathrm{~km}$; Varuna, $D \sim 900 \mathrm{~km}$ ).

The formation of these reservoirs has been accompanied by substantial scattering of comets towards the zone of the terrestrial planets; these comets possibly supply carbon and organics to their surfaces (see Sect. 8.5) but probably make only a limited contribution to Earth's water (see Chap. 2, Part I by Petit and 
Morbidelli). The composition of the comets coming from the two reservoirs is expected to differ more or less strongly according to the various formation models. Understanding the formation scenario of comets is vital for any considerations on the ubiquity (or scarcity) of terrestrial planets favourable to life development elsewhere in the Universe.

\subsubsection{The Active Comet}

When a comet approaches the Sun, it enters the active phase. A coma develops around the nucleus, which consists of a roughly spherical halo of neutral gas and dust particles. The radius of the coma depends on the chemical species, and ranges between less than a thousand to more than one million $\mathrm{km}$ (e.g. Crovisier and Encrenaz, 2000) (Fig. 8.2). What we call here "radius" is indeed a characteristic length describing the progressive decrease of the spatial density of particles. Beyond this coma, an atomic hydrogen cloud is produced by the photodissociation of a number of species, mainly $\mathrm{H}_{2} \mathrm{O}$ and $\mathrm{OH}$; this cloud can reach 1 to 10 million $\mathrm{km}$. Starting from the nucleus and the coma, three types of cometary tails develop: a plasma tail, a dust tail, and a tail of neutral sodium

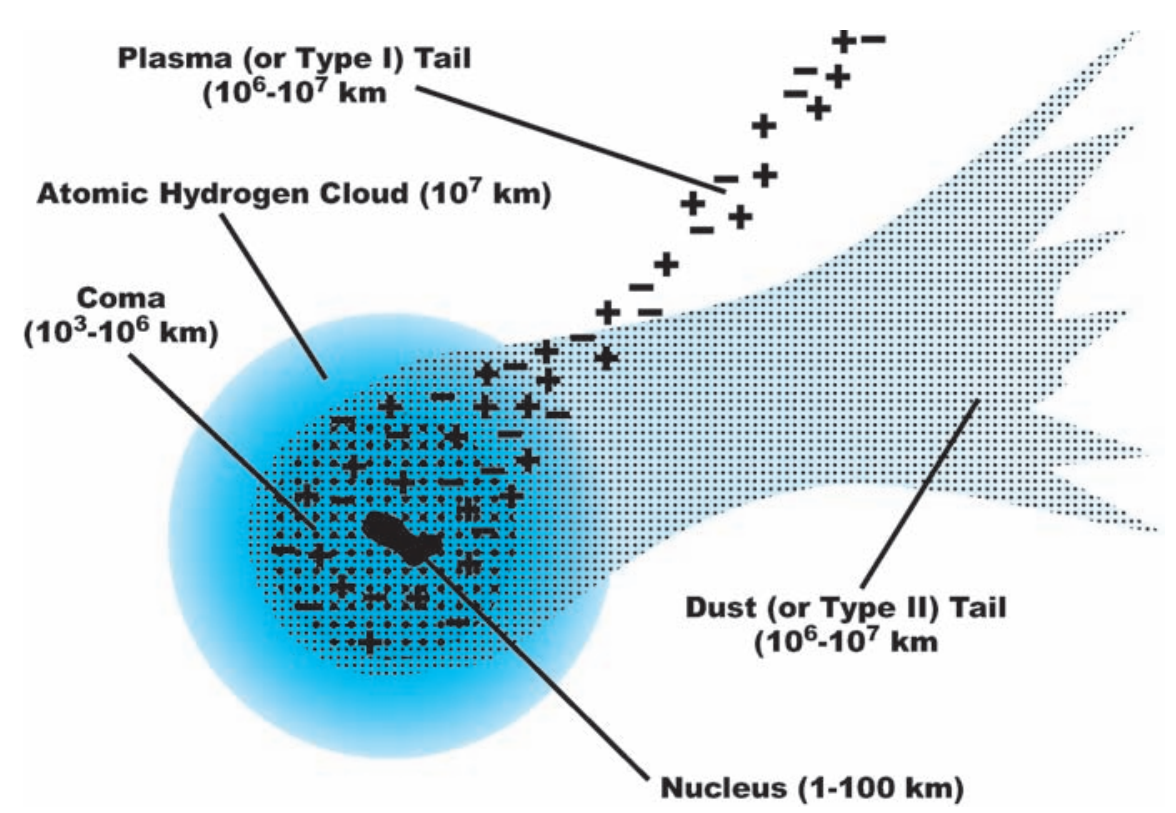

Fig. 8.2. Schematic view of an active comet. The Sun is (approximately) in the direction opposite to the ion tail, whose shape is due to the interaction with the rapid ( $\sim 400-800 \mathrm{~km} / \mathrm{s}$ ) charged particles of the solar wind. The dust particles that were previously released from the nucleus are slowly pushed away by the pressure of the solar photons, which, combined with the comet motion (here toward the left) explains the shape of the dust tail (see e.g. Festou et al., 1993b) 
atoms - the latter clearly seen recently in observations of comet Hale-Bopp (Cremonese et al., 1997).

The plasma tail is made of (atomic and molecular) ions and electrons. It has a characteristic blue colour due to the $\mathrm{CO}^{+}$ions, a very rectilinear shape and is almost perfectly opposed to the Sun's direction. It is produced by the interaction of the solar wind - itself made of protons and charged particles - with the ions produced in the coma (Fig. 8.3). The yellow-white dust tail consists of small grains that have typically a diameter between 1 and 10 micrometres, and that reflect and scatter the light of the Sun. Its shape depends on the balance between the gravitational force from the Sun, and the radiation pressure due the photons of the solar radiation.

An antitail is seen in some comets, looking like a narwhal tusk pointing toward a direction opposite to the other tails. It is in fact always present, but not always visible. It reveals the presence of an accumulation of particles in the orbital plane of the comet. These particles are larger than those of the normal dust tail, and have thus been ejected at low speed from the cometary nucleus (they are less easily lifted by the gas flow) and are less sensitive to solar-radiation pressure (their mass/surface ratio is larger). By a projection effect, these particles appear as a spike at the head of the comet, when the Earth happens to cross the comet orbital plane. This feature usually appears only on the Sun side, but

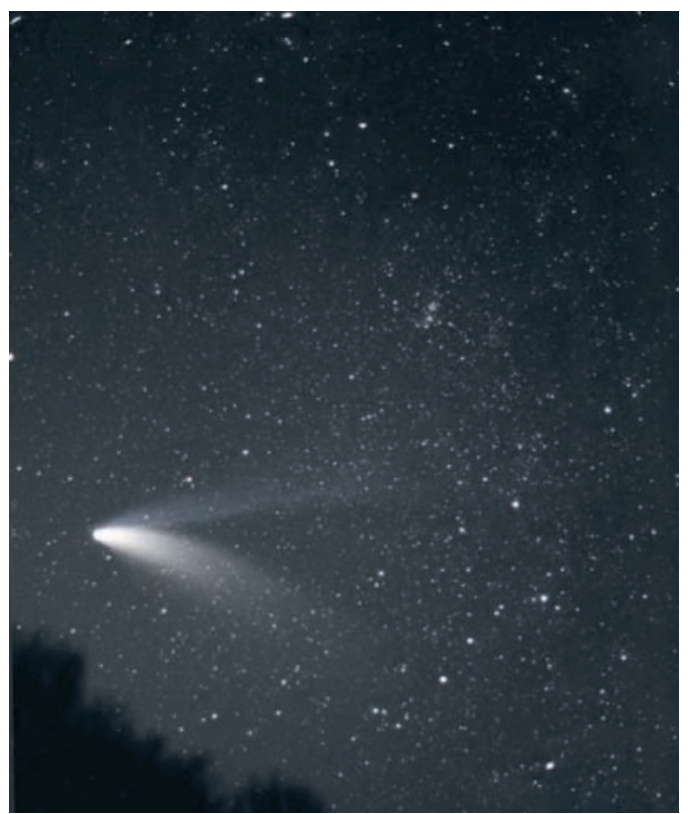

Fig. 8.3. Image of a comet with well-developed tails (Hale-Bopp). The plasma tail (thin, rectilinear and bluish) is clearly recognized from the dust tail (diffuse, curved and white-yellow). (C) Photo R. Lauqué) 


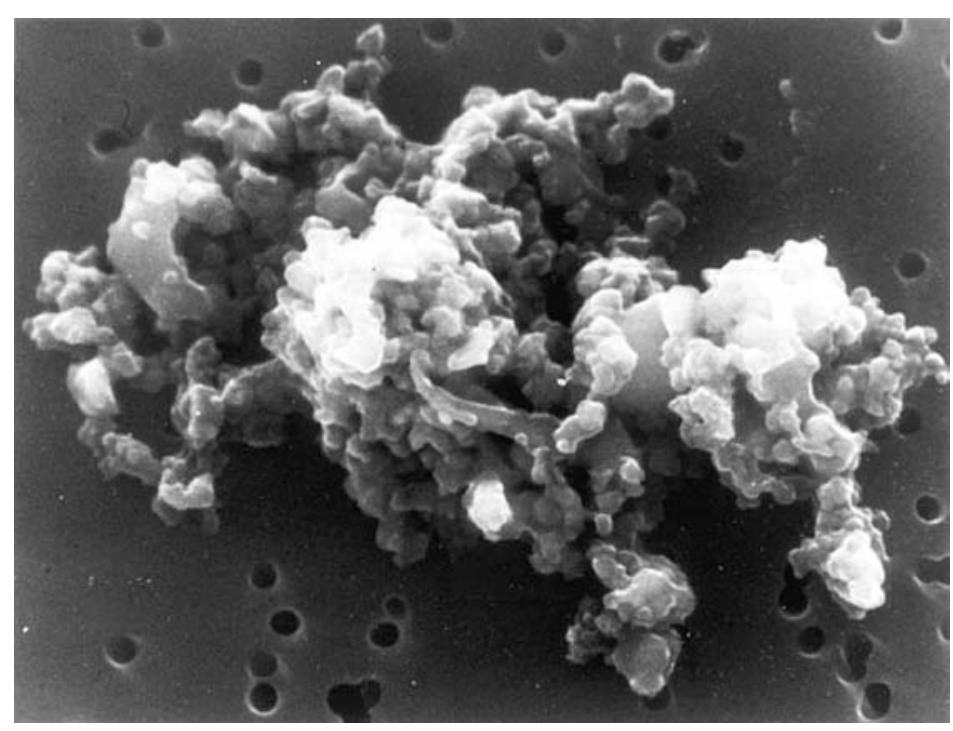

Fig. 8.4. Brownlee particle (interplanetary dust collected in the stratosphere). For the fluffiest of these particles, the origin is probably cometary

can sometimes also be distinguished as a thin line in the antisolar direction, superposed to the normal dust tail. The "neck-line" recently observed on comet $\mathrm{P} /$ Churyumov-Gerasimenko is an antitail (Weiler et al., 2004).

Cometary dust particles disperse more or less rapidly into the interplanetary space, according to their shape, nature and initial velocity. Some of them cross the path of the Earth; if large enough they produce meteors. One can get an idea of cometary dust particles by looking at particles collected in the Earth stratosphere ("Brownlee particles", or stratospheric interplanetary dust particles - SIDP). Many of these particles have a large surface to mass ratio and a very irregular, and somewhat fractal-like structure (Fig. 8.4). All collected particles are, however, not as irregular: one finds also among SIDPs more compact particles, similar to small blocks of clay. It is tempting - but perhaps too simplistic - to attribute to the most irregular particles a cometary origin, whereas the most compact ones would come from asteroids.

The third cometary tail, the sodium tail, made of neutral sodium atoms, was discovered for the first time in the Great Comet C/1910 A1, and reobserved more recently in comets C/1957 P1 (Mrkos) and Hale-Bopp. Sodium is pushed away in the antisolar direction by the radiation pressure, in a way similar to what happens for dust particles.

\subsubsection{Gas and Dust Production}

The weakest $\mathrm{H}_{2} \mathrm{O}$ production rates currently detected in comets are of $10^{26}$ molecules $/ \mathrm{s}(3 \mathrm{~kg} / \mathrm{s})$ when the comet is favourably situated. In comet Hale-Bopp 
at perihelion, the production rate reached up to $10^{31}$ molecules/s, which corresponds to 300 tons/s. These rates imply that a bright comet typically loses a 1-m thick sheet at each perihelion passage. Thus for a nucleus of about a few tens $\mathrm{km}$ in diameter, one expects the comet to disappear after a few thousand passages close to the Sun. This implies that today short-period comets $(P<200 y r)$ cannot have occupied their present orbit since the beginning of the Solar System.

The expansion speed of most molecules in the coma is about $1 \mathrm{~km} / \mathrm{s}$. Photodissociation reactions take place, for example $\mathrm{H}_{2} \mathrm{O} \rightarrow \mathrm{H}+\mathrm{OH}$, followed by $\mathrm{OH} \rightarrow \mathrm{O}+\mathrm{H}$. For the photodissociation fragments, the speeds can reach several $\mathrm{km} / \mathrm{s}\left(8\right.$ and $21 \mathrm{~km} / \mathrm{s}$ in the case of $\mathrm{H}$ produced from $\left.\mathrm{H}_{2} \mathrm{O}\right)$. The matter ejected by comets is almost equally distributed in mass between volatiles (mainly $\mathrm{H}_{2} \mathrm{O}$ ) and dust - within a factor 10. It is possible that the dispersion of the gas/dust ratio between comets represents a variation in the initial composition of the cometary nucleus; alternatively, it can also be an effect of evolution after many passages close to the Sun.

The dark surface observed on comet Halley's nucleus may consist of an accumulation of dust, and/or of complex refractory organic molecules. This crust may be broken by the explosion of gas pockets heated under the crust: this would enrich the coma in dust particles, and appear as an outburst of the comet.

\subsubsection{Remote Activity, Outbursts and Split Comets}

The activity of comets strongly depends on the distance to the Sun. It has long been thought that cometary activity developed only when the comet was closer than 4AU from the Sun, because the "dirty snow ball" model of the nucleus suggested that water, the major volatile, controlled the emission of other molecules and of solid particles. The sublimation of $\mathrm{H}_{2} \mathrm{O}$ ice is important only at heliocentric distances smaller than $4 \mathrm{AU}$ and drives the coma development for such distances. However, a preperihelion cometary activity was detected in comet Hale-Bopp as far as 7 AU from the Sun. This activity has been attributed to the sublimation of carbon monoxide. $\mathrm{CO}$ is indeed present in large quantities with respect to $\mathrm{H}_{2} \mathrm{O}$, from $5 \%$ to above $20 \%$ - depending on the authors, the measurement techniques and the circumstances (type of comet, distance to the Sun). CO outgassing can, by a process similar to $\mathrm{H}_{2} \mathrm{O}$ outgassing, lift dust particles and create a coma (Bockelée-Morvan and Rickman, 1999). After its passage at perihelion, comet Hale-Bopp was still outgassing $\mathrm{CO}$ as far as $14 \mathrm{AU}$.

Cometary matter is usually divided into two components: "refractories" and "volatiles"; the word "refractories" applies to species that do not sublimate. This classification is sometimes refined, introducing "hypervolatiles" (Ar, $\left.\mathrm{N}_{2}, \ldots\right)$, and "semirefractories" (e.g. organic refractories, as opposed to more refractory species like silicates). Note here that water, traditionally ranking as the major volatile species in comets, behaves at $7 \mathrm{AU}$ like a refractory. 
A peculiar comet, 29P/Schwassmann-Wachmann 1, which follows an almost circular orbit at $6 \mathrm{AU}$ from the Sun (i.e. somewhat beyond Jupiter), harbours intense and unpredictable outbursts; here also, CO is at the origin of this activity. Comet Halley showed an activity outburst after its perihelion passage, when it was already $14 \mathrm{AU}$ from the Sun. Because of the lack of spectroscopic information, one does not know for sure if this outburst was also due to CO; on the pictures, only the development of a coma was noticed.

Breakup of cometary nuclei (see Boehnhardt 2002) is not uncommon. Some comets break into a few pieces (C/1975 v1 West), some into many pieces $(57 \mathrm{P} / \mathrm{du}$ Toit-Neujmin-Delporte in 2002), some even vanish (C/1999 S4 LINEAR). 90\% of the Sun-grazing comets observed by the SOHO satellite belong to same orbital family: Kreutz family (Lamy and Biesecker 2002). They may have their origin in the splitting of one large comet. Depending on their size, the fragments have a lifetime between hours and years. The rate of splitting events of periodic comets has been evaluated to 0.01/yr/comet (Chen and Jewitt 1994). This makes splitting an important mass-loss factor, comparable to ordinary activity, and an important cause of comet death. While tidal forces close to Jupiter were clearly responsible for the breakup of Shoemaker-Levy 9, the other cases of splitting can occur at any place along the orbit, generally far from any planet. Other explanations (beside tidal forces - when applicable) involve thermal stress and gas explosions, possibly linked to runaway exothermic transformation of amorphous to crystalline ice, rotational breakup due to centrifugal force (Jewitt 2002) and fragmentation due to a collision with another small Solar System object.

Splitting events are an important source of information on the internal structure of comets. During the complete splitting of C/1999 S4 LINEAR, BockeléeMorvan et al., (2001) detected no change in the relative molecular abundances, which pleads in favour of a rather homogeneous composition of the nucleus. Encounters with split cometary nuclei are a likely explanation for impact-crater chains seen on giant planet satellites, and that probably occurred on Earth too.

\subsubsection{Nucleus Modelling: Outgassing and Internal Temperatures}

Detailed modelling of the physical processes involved in nucleus formation, evolution and outgassing is a key tool to address important issues such as the pristine character of cometary matter, and the relation between abundances in the nucleus and those measured in the coma (see Sect. 8.2.1.4). Important phenomena and comet parameters include: ice sublimation; porosity of the cometary matter, which decreases from small to large objects, contributing to the density variation of icy bodies (from $\sim 0.5 \mathrm{~g} / \mathrm{cm}^{3}$ for small comets to $2 \mathrm{~g} / \mathrm{cm}^{3}$ for Pluto); heat transport by sublimated gases in the pores, which usually dominates solid thermal conductivity; radioactive heating by long-period elements like ${ }^{235} \mathrm{U},{ }^{238} \mathrm{U},{ }^{40} \mathrm{~K}$; disruptive and shattering collisions between comets; amorphous to crystalline ice transition, with possible release of trapped gas; surface 
processing by solar and galactic photons and particles. In the formation phase, heat from the short-period element ${ }^{26} \mathrm{Al}$ and heat due to accretion itself may be critical, but their role and magnitude vary greatly from one model to another. The difference between the production rates of $\mathrm{CO}$ (highly volatile) and $\mathrm{H}_{2} \mathrm{O}$ as a function of distance to the Sun is well reproduced by models (e.g. Prialnik 1999, 2002, Enzian 1999, Coradini et al., 1997a,b, Capria 2002, Benkhoff 1999, Huebner and Benkhoff 1999a); a review of those models is presented by Huebner et al., (1999b). However, several other phenomena take place that lead to the observed abundance in the coma; they make the link between nucleus and coma abundances for some species more complex $\left(\mathrm{HNC}, \mathrm{H}_{2} \mathrm{CO}, \ldots\right)$, whereas not preventing in other cases a simple connection, as shown by species whose release closely follows that of $\mathrm{H}_{2} \mathrm{O}$, without significant effects of differential sublimation (see Sect. 8.2.1.4 and Fig. 8.5).

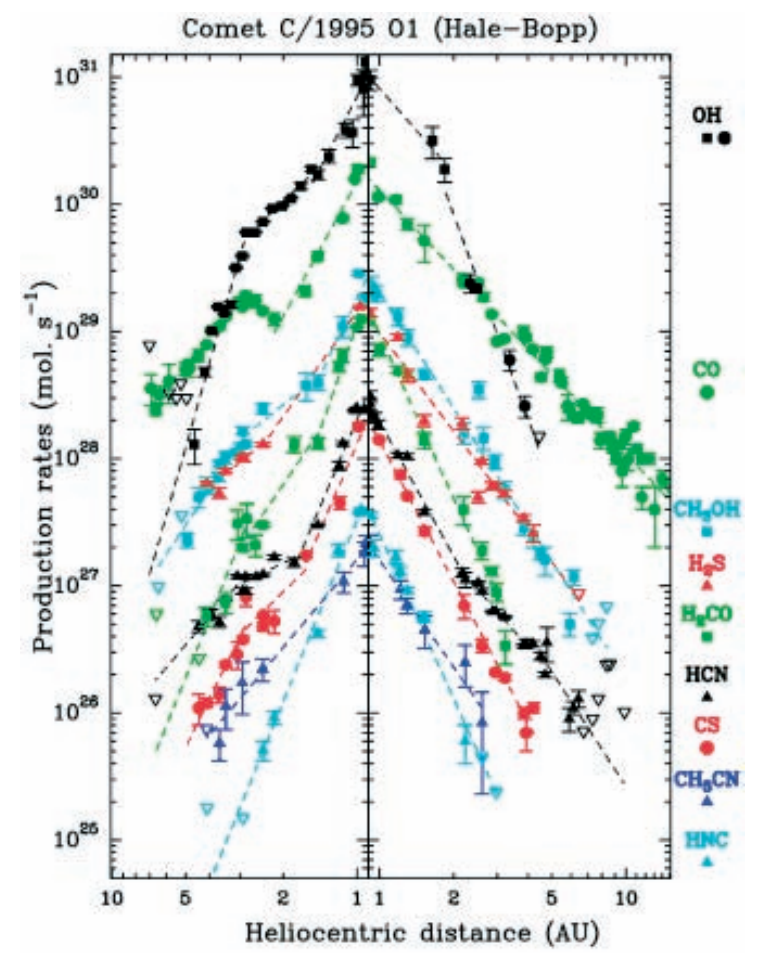

Fig. 8.5. This diagram displays the production rate $Q_{\mathrm{x}}$ of various cometary molecules as a function of heliocentric distance $r_{\mathrm{H}}$ (Biver et al. 2002). In this logarithmic scale $Q_{\mathrm{X}}$ shows, in general, a linear trend, corresponding to a power law in $r_{\mathrm{H}}$. Whereas some species clearly depart from the mean behaviour ( $\mathrm{HNC}, \mathrm{H}_{2} \mathrm{CO}, \mathrm{CO}, \mathrm{CS}$ - see discussion in text), the variation of the remaining species (e.g. $\mathrm{CH}_{3} \mathrm{OH}, \mathrm{HCN}, \mathrm{H}_{2} \mathrm{~S}, \mathrm{CH}_{3} \mathrm{CN}$ ) are roughly parallel $\left(\sim r_{\mathrm{H}}^{-2}\right)$, which sets a limit on the importance of differential sublimation effects for these species 


\subsubsection{Internal Temperature and the Case for Liquid Water}

A crucial issue for prebiotic chemistry and exobiology is the maximum temperature reached by cometary matter during its history. Prialnik and Podolak (1999) consider that comets form rapidly, which leads to a key role of ${ }^{26} \mathrm{Al}$ decay in heating the interior of that comet. The maximal temperature reached somewhere in the nucleus ranges from low $(35-90 \mathrm{~K}$ for a small $1-\mathrm{km}$ radius comet with low/ high $(0.1 / 0.9)$ porosity) to $270-210 \mathrm{~K}$ for a $100-\mathrm{km}$ radius object. A very different situation is found in McKinnon (2002), who modelled the thermal history of large icy bodies in the outer solar nebula (Kuiper Belt). The accretion there is slow enough for ${ }^{26} \mathrm{Al}$ to have mostly decayed, and collisional accretion is the main heat source. The temperature remains below $100 \mathrm{~K}$ in most places for radii below $100 \mathrm{~km}$, but reaches more than $250 \mathrm{~K}$ in the case of KBO Varuna $(R \sim 400 \mathrm{~km})$.

The prospect for high temperature inside the nucleus raises the question of possible liquid water inside comets or Kuiper Belt objects. Liquid water is a key parameter for chemical evolution toward prebiotic molecules, such as amino acids more complex than glycine. Therefore liquid water events on comets might allow the synthesis of more molecules than those one would expect in "dry" comets. From the model quoted above, liquid water seems excluded at present times in the bodies of modest size $(<100 \mathrm{~km}$ diameter $)$ reaching the zone of terrestrial planets. Conditions were more favourable in the past but only a very limited parameter range would allow liquid water to be present for some time (Podolak and Prialnik 1997); in the case of KBOs, the body should be among the largest (Pluto?).

\subsection{Chemical Composition of Comets as Deduced from Observations}

\subsubsection{Volatiles}

\subsubsection{Observations}

Many observations and new discoveries concerning cometary volatiles were made recently, particularly thanks to the development of new instruments (large millimetre-wavelength radio telescopes, among which the IRAM 30-m dish on Pico Veleta and the Plateau de Bure interferometer have proven especially useful; infrared instruments, either ground-based or space-borne: Keck Telescope with NIRSPEC, and ISO satellite) and with the presence of very active comets: Hyakutake in 1996 and Hale-Bopp in 1997 (Bockelée-Morvan 1997, Despois 1999, Crovisier 1999, Bockelée-Morvan et al., 2000). Ten years before, the passage of comet Halley in 1985-86 had already brought considerable progress, from ground-based observations as well as from space probes, in particular Giotto and Vega 1 and 2 (Crovisier and Schloerb 1991, Encrenaz and Knacke 1991). 
The majority of new detections of parent molecules (i.e. molecules coming directly from the nucleus) have been performed through radioastronomical observations. This technique provides very safe identifications of the molecular species through very high resolution study $\left(R=\lambda / \Delta \lambda \sim 10^{7}\right.$ or better) of their rotational spectrum. Molecular abundances from radio data are usually considered to be accurate within 10-20\% (calibration uncertainties), provided the excitation model for the molecule is correct. When comparing various techniques (UV, IR, radio), abundances agree usually well (within a factor of 2 at most). Among the species detected for the first time in Hale-Bopp, formamide $\left(\mathrm{NH}_{2} \mathrm{CHO}\right)-$ a species already complex for astronomers! -already bears some resemblance to glycine $\mathrm{NH}_{2}-\mathrm{CH}_{2}-\mathrm{COOH}$.

\subsubsection{Molecular Abundances in Cometary Ices}

Molecules detected in comets are given in Table 8.1; abundances - by molecule number - are expressed in per cent with respect to $\mathrm{H}_{2} \mathrm{O}$. The majority of the values are from Hale-Bopp observations. The next columns indicate, where available, the comet-to-comet variation range, and the number of comets observed (detected + undetected; Biver et al., 2002). The meaning of these abundances is discussed below (Sect. 8.2.1.4).

The most abundant species, with abundances around $10 \%$ of $\mathrm{H}_{2} \mathrm{O}$, are carbon monoxide $\mathrm{CO}$ (from 1 to $30 \%$, see below), carbon dioxide $\mathrm{CO}_{2}(5 \%)$ and methanol $(1-5 \%)$. At the $\sim 1 \%$ level one finds the first sulfur-bearing species, $\mathrm{H}_{2} \mathrm{~S}$, formaldehyde $\mathrm{H}_{2} \mathrm{CO}$ (its exact abundance is hard to determine, due to the extended source problem discussed below), and the first nitrogen-bearing species: ammonia $\mathrm{NH}_{3}(0.6 \%)$. Then, three hydrocarbons that are observable only in the infrared: methane $\mathrm{CH}_{4}$ and ethane $\mathrm{C}_{2} \mathrm{H}_{6}(0.6 \%)$ and acetylene $\mathrm{C}_{2} \mathrm{H}_{2}(0.1-0.5 \%)$. With $0.25 \%$, ethylene glycol $\mathrm{CH}_{2} \mathrm{OHCH}_{2} \mathrm{OH}$ is the second alcohol. One also finds at these levels other sulfur species like $\mathrm{SO}_{2}(0.1 \%)$, OCS and $\mathrm{CS}_{2}$, and $\mathrm{HCN}$ $(0.2 \%)$. SO $(0.2-0.8 \%)$ is probably mostly a product of $\mathrm{SO}_{2}$ photodissociation (estimated itself around $0.1 \%$ ). The least abundant currently detectable species have a relative abundance of approximately $10^{-4}$. Among them, isocyanic acid $\mathrm{HNCO}$, formic acid $\mathrm{HCOOH}$, the disulfur $\mathrm{S}_{2}$, methylformate $\mathrm{HCOOCH}_{3}, \mathrm{HNC}$ (see below), methyl cyanide $\mathrm{CH}_{3} \mathrm{CN}$ and cyanoacetylene $\mathrm{HC}_{3} \mathrm{~N}$ (in comparable abundances), thioformaldehyde $\mathrm{H}_{2} \mathrm{CS}$ and formamide $\mathrm{NH}_{2} \mathrm{CHO}$.

\subsubsection{A Few Important Cases}

\section{- Newly Found Species}

Bockelée-Morvan et al., (2000, note), and Bockelée-Morvan and Crovisier (2002) report the identification of acetaldehyde $\mathrm{CH}_{3} \mathrm{CHO}$, with an abundance of about $0.02 \%$ relative to $\mathrm{H}_{2} \mathrm{O}$ from a reanalysis of comet Hale-Bopp data. Recently, $\mathrm{CH}_{2} \mathrm{OCH}_{2} \mathrm{OH}$ (ethylene glycol - commonly "antifreeze") has been identified 
Table 8.1. Relative production rates of molecules in the coma, expressed by molecule number and in \% relative to water ice (adapted from Despois et al., 2002). This rates are believed to be good tracers of molecular abundances in cometary ices, except for some species, like $\mathrm{CO}, \mathrm{H}_{2} \mathrm{CO}, \mathrm{HNC}$ (see text). The values are for comet Hale-Bopp (Bockelée-Morvan et al., 2000, Crovisier et al., 2004a,b), except when indicated Hya. (Hyakutake) or I-Z (Ikeya-Zhang). When the molecule has been measured in several comets a range (Biver et al., 2002) is given

\begin{tabular}{|c|c|c|c|c|}
\hline Molecule & $\begin{array}{l}\text { Abundance } \\
\left(\mathrm{H}_{2} \mathrm{O}=100\right)\end{array}$ & $\begin{array}{l}\text { Intercomet } \\
\text { variation }\end{array}$ & $\begin{array}{l}\text { Detected comets } \\
+ \text { upper limits }\end{array}$ & Notes \\
\hline $\mathrm{H}_{2} \mathrm{O}$ & 100 & & & \\
\hline $\mathrm{H}_{2} \mathrm{O}_{2}$ & $<0.03$ & & & \\
\hline $\mathrm{CO}$ & 23 & $<1.7-23$ & $5+4$ & Var. \\
\hline $\mathrm{CO}_{2}$ & 6 (various) & & & \\
\hline $\mathrm{CH}_{4}$ & 0.6 & & & \\
\hline $\mathrm{C}_{2} \mathrm{H}_{6}$ & 0.6 & & & \\
\hline $\mathrm{C}_{2} \mathrm{H}_{2}$ & 0.2 & & & \\
\hline $\mathrm{C}_{4} \mathrm{H}_{2}$ & $0.05 ?(\mathrm{I}-\mathrm{Z})$ & & & Prelim. \\
\hline $\mathrm{CH}_{3} \mathrm{C}_{2} \mathrm{H}$ & $<0.045$ & & & \\
\hline $\mathrm{CH}_{3} \mathrm{OH}$ & 2.4 & $<0.9-6.2$ & $15+2$ & \\
\hline $\mathrm{H}_{2} \mathrm{CO}$ & 1.1 & $0.13-1.3$ & $13+2$ & Extend \\
\hline $\mathrm{CH}_{2} \mathrm{OHCH}_{2} \mathrm{OH}$ & 0.25 & & & \\
\hline $\mathrm{HCOOH}$ & 0.09 & & & \\
\hline $\mathrm{HCOOCH}_{3}$ & 0.08 & & & \\
\hline $\mathrm{CH}_{3} \mathrm{CHO}$ & 0.025 & & & \\
\hline $\mathrm{H}_{2} \mathrm{CCO}$ & $<0.032$ & & & \\
\hline $\mathrm{c}-\mathrm{C}_{2} \mathrm{H}_{4} \mathrm{O}$ & $<0.20$ & & & \\
\hline $\mathrm{C}_{2} \mathrm{H}_{5} \mathrm{OH}$ & $<0.10$ & & & \\
\hline $\mathrm{CH}_{2} \mathrm{OHCHO}$ & $<0.04$ & & & \\
\hline $\mathrm{CH}_{3} \mathrm{OCH}_{3}$ & $<0.45$ & & & \\
\hline $\mathrm{CH}_{3} \mathrm{COOH}$ & $<0.06$ & & & \\
\hline $\mathrm{NH}_{3}$ & 0.7 & & & \\
\hline $\mathrm{HCN}$ & 0.25 & $0.08-0.25$ & $24+0$ & \\
\hline $\mathrm{HNCO}$ & 0.10 & & & \\
\hline $\mathrm{HNC}$ & 0.04 & $<0.003-0.035$ & $5+2$ & Extend \\
\hline $\mathrm{CH}_{3} \mathrm{CN}$ & 0.02 & $0.013-0.035$ & $4+0$ & \\
\hline $\mathrm{HC}_{3} \mathrm{~N}$ & 0.02 & & & \\
\hline
\end{tabular}


Table 8.1. (continued)

\begin{tabular}{|c|c|c|c|c|}
\hline Molecule & $\begin{array}{l}\text { Abundance } \\
\left(\mathrm{H}_{2} \mathrm{O}=100\right)\end{array}$ & $\begin{array}{l}\text { Intercomet } \\
\text { variation }\end{array}$ & $\begin{array}{l}\text { Detected comets } \\
+ \text { upper limits }\end{array}$ & Notes \\
\hline $\mathrm{NH}_{2} \mathrm{CHO}$ & 0.015 & & & \\
\hline $\mathrm{NH}_{2} \mathrm{OH}$ & $<0.25$ & & & \\
\hline $\mathrm{HCNO}$ & $<0.0016$ & & & \\
\hline $\mathrm{CH}_{2} \mathrm{NH}$ & $<0.032$ & & & \\
\hline $\mathrm{NH}_{2} \mathrm{CN}$ & $<0.004$ & & & \\
\hline $\mathrm{N}_{2} \mathrm{O}$ & $<0.23$ & & & \\
\hline Glycine I & $<0.15$ & & & \\
\hline $\mathrm{C}_{2} \mathrm{H}_{5} \mathrm{CN}$ & $<0.010$ & & & \\
\hline $\mathrm{HC}_{5} \mathrm{~N}$ & $<0.003$ & & & \\
\hline $\mathrm{H}_{2} \mathrm{~S}$ & 1.5 & $0.12-1.5$ & $11+3$ & \\
\hline OCS & 0.4 & & & Extend. \\
\hline $\mathrm{SO}$ & 0.3 & & & Extend. \\
\hline $\mathrm{CS}_{2}$ (from CS) & 0.17 & $0.05-0.17$ & $9+0$ & \\
\hline $\mathrm{SO}_{2}$ & 0.2 & & & \\
\hline $\mathrm{H}_{2} \mathrm{CS}$ & 0.02 & & & \\
\hline $\mathrm{S}_{2}$ & 0.005 (Нya) & & & \\
\hline $\mathrm{CH}_{3} \mathrm{SH}$ & $<0.05$ & & & \\
\hline NS & 0.02 & & & Parent? \\
\hline $\mathrm{PH}_{3}$ & $<0.16$ & & & \\
\hline $\mathrm{NaOH}$ & $<0.0003$ & & & \\
\hline $\mathrm{NaCl}$ & $<0.0008$ & & & \\
\hline
\end{tabular}

Notes (see text for discussion):

- Extend.: extended source - in this case the abundance depends somewhat on the model adopted for the spatial distribution of the source

- Var.: CO shows large variability in a given comet along the orbit, and from comet to comet

- $\mathrm{C}_{4} \mathrm{H}_{2}$ values are preliminary

- NS is probably a photodissociation product from an unknown parent.

- $\mathrm{CS}_{2}$ abundance indirectly deduced from CS abundance

- $\mathrm{CO}_{2}$ measured to be $20 \%$ in Hale-Bopp at a distance of 2.9 UA by the ISO satellite

- Glycine is a compound with a very low volatility. Nondetection in the gaseous phase does not imply absence from the refractory component. Glycine I refers to one of the two most stable conformers of the molecule. 
from the same archive spectra (Crovisier et al., 2004b); surprisingly, its abundance $(0.25 \%)$ is higher than the upper limit for ethanol $(0.1 \%)$, which may be an important constraint on the formation processes of cometary molecules. $\mathrm{C}_{4} \mathrm{H}_{2}$ has been possibly identified in the near infrared (NIR) spectrum of comet C/2002 C1(Ikeya-Zhang) (Magee-Sauer 2002). NS $(x \sim 0.02 \%)$, a radical, probably a dissociation product, has been detected in comet Hale-Bopp (Irvine et al., 2000 b); its parent is not yet known: $\mathrm{HNCS}, \mathrm{HNSO}, \mathrm{NH}_{4} \mathrm{SH}$ have been suggested.

- Supervolatiles

Very volatile ("supervolatile") species, like Ar, $\mathrm{N}_{2}, \mathrm{H}_{2}$, $\mathrm{CO}$ are important to set an upper limit to the highest temperature ever reached by the cometary nucleus and thus its degree of thermal processing. Bockelée-Morvan and Crovisier (2002) report doubts on the detection of $\mathrm{Ar}$ and $\mathrm{N}_{2}$ (from $\mathrm{N}_{2}{ }^{+}$). $\mathrm{H}_{2}$ appears to be a product of water dissociation, not a nuclear species. To date, the most volatile species are thus $\mathrm{CO}$ and $\mathrm{CH}_{4}$.

\section{- Glycine Upper Limit in Volatiles}

Table 8.1 includes upper limits from radio observations of the coma (BockeléeMorvan and Crovisier 2002). The limit $(x<0.15 \%$ or $\sim 0.004$ by weight or $6 \times 10^{-5} \mathrm{~mol} / \mathrm{g}$ ) on glycine (the simplest amino acid, and one of the 20 that are the building blocks of proteins) is not very stringent, due to the intrinsic weakness of its radio lines. Furthermore, this limit concerns only the fraction of glycine molecules that could be released from the nucleus due to ice sublimation. Note that pure solid glycine itself is a refractory compound $\left(T_{\text {subl }}\right.$ is $\sim 500 \mathrm{~K}$ for $1 \mathrm{~atm}$ pressure) and that thermal destruction occurs at a similar temperature. In the CM carbonaceous chondrite meteorite Murchison, glycine abundance (here with respect to refractories) is about $10^{-7} \mathrm{~mol} / \mathrm{g}$ (Cronin and Chang 1993).

\subsubsection{Are Coma Abundances a True Image of Ice Composition in the Nucleus?}

On a plot of their production rates according to the distance to the Sun (Fig. 8.5 "Christmas tree plot" Biver et al., 2002), a majority of individual molecules vary in a similar way, which suggests that most of the cometary material escapes as a whole, without strong differentiation between chemical species. A few species (most notably $\mathrm{CO}, \mathrm{H}_{2} \mathrm{CO}$, and $\mathrm{HNC}$ ) harbour, however, a peculiar behaviour, and require additional phenomena to be taken into account: differential sublimation, extended source, and coma chemistry.

\section{- Differential Sublimation}

The great volatility of $\mathrm{CO}$ induces an important variation of the $\mathrm{CO} / \mathrm{H}_{2} \mathrm{O}$ ratio with heliocentric distance. $\mathrm{CO}$ can not only move through the micropores present in the ice, but even through the structure of crystalline ice itself; as a result, the surface layers of the nucleus can, according to cases, be either depeleted or enriched in CO. This phenomenon has been modelled in detail (see Sect. 8.1.7). 


\section{- Extended Sources}

Some coma molecules do not appear to be emitted directly from the nucleus, but are more probably secondary products of photo- or thermal degradation of larger species, or products of chemical reactions in the coma. Thus they appear to come from an extended source surrounding the nucleus. Bockelée-Morvan and Crovisier (2002) summarise different indicators of extended source (spatial distribution, line shape, production behaviour with distance to the Sun). The best established cases are $\mathrm{H}_{2} \mathrm{CO}$ and $\mathrm{CO}$. Recent observations indicate that $\mathrm{SO}$, CS, OCS and HNC harbour extended source characteristics. Photodissociation is likely to explain the case of $\mathrm{SO}$ (from $\mathrm{SO}_{2}$ ) and $\mathrm{CS}$ (from a yet uncertain parent, maybe $\mathrm{CS}_{2}$ ). HNC may be partly chemically produced (see below). The origin of $\mathrm{H}_{2} \mathrm{CO}$ is not only in the nucleus, but also in the coma, where it is released from a "carrier": maybe organic CHON grains, maybe polymers (polyoxymethylene - POM, which has been detected after experimental simulations of cometary ice analogs), maybe both. The degradation of polyoxymethylene (POM) has been studied in the laboratory and is found to be a possible source for $\mathrm{H}_{2} \mathrm{CO}$ (Cottin et al., 2001, 2004). Various CN-bearing compounds including hexamethylenetetramine (HMT, see Fig. 8.13) and poly-HCN are possible sources of extended $\mathrm{CN}$ radical in the coma (Fray et al., submitted) in addition to the photodissociation of HCN.

\section{- Coma Chemistry}

$\mathrm{HNC}$ is a case for which coma reactions (other than photodissociation) can affect molecular abundances. The peculiar behaviour of the production rate of this species in comet Hale-Bopp, with its strong increase when the comet approached the sun, leads to the proposal of its production from HCN through chemical reactions in the coma as an explanation. Rodgers and Charnley (2001a) shows that reactions between HCN and high-speed ("suprathermal") hydrogen (from $\mathrm{H}_{2} \mathrm{O}$ dissociation) could explain HNC production in comet Hale-Bopp. This process, however, is not efficient for smaller comets such as comet Hyakutake, for which the origin of HNC is still an open question (Irvine et al., 2003, Rodgers and Charnley 2001, Charnley et al., 2002a,b). It should be noted that in interstellar clouds, and thus perhaps also in protosolar nebulae, HNC and HCN are both present, and that HNC is stable in ice at sufficiently low temperature. Therefore, the presence of some HNC in the nucleus is not excluded.

Are some other minor species due to reactions in the coma? Rodgers and Charnley (2001b) show it cannot be the case for the detected species $\mathrm{HCOOCH}_{3}$ and $\mathrm{HCOOH}$, which are then expected to be directly released from the nucleus.

\subsubsection{Grains}

\subsubsection{Nature of Grains}

The chemical composition of the grains of comet Halley has been analyzed in situ by mass spectrometry (instrument PUMA on board the Vega $1 \& 2$ space- 
craft, and instruments PICCA, IMS and NMS on board Giotto spacecraft - Altwegg et al., 1999). When grains are ejected from the nucleus, any embedded ice sublimes within the first few kilometres. Therefore, we will only consider the refractory (nonvolatile) component of grains in this section. Vega spacecraft have collected particles between 80000 and $120000 \mathrm{~km}$ from the nucleus. About 5000 particles were analyzed, with mass ranging from $5 \times 10^{-17}$ to $5 \times 10^{-12} \mathrm{~g}$ (which means a size ranging from 0.04 to $2 \mu \mathrm{m}$ if one assumes a density equal to 1 ). The total mass that was analyzed was only a few nanograms. Their elemental composition shows that the particles are made of a mixture of silicates and organic material. In comet Halley's coma, when Giotto made its measurements, the ratio between silicates and organic matter was found to be between 1 and 2 . When organic matter is very abundant, the grains are called "CHON" grains (i.e., compounds of $\mathrm{C}, \mathrm{H}, \mathrm{O}$ and $\mathrm{N}$ ). According to some models, the small mineral particles might be embedded in a matrix of carbonaceous matter (Greenberg 1982); however, mass spectrometer measurements show the existence of pure silicate an $\mathrm{d}$ pure organic grains, beside grains of mixed composition (Fomenkova, 1999).

Figure 8.6 shows the production of grains in mass and number for comet Halley as derived by Crifo (Crifo 1995, Crifo and Rodionov 1997) from in situ measurements presented in McDonnell et al. (1991). It must be noted that data up to $1 \mathrm{~mm}$ are direct in situ measurements, whereas higher values are extrapolations (relevant within an order of magnitude of uncertainty).

The temperature of grains can be derived from observations, but the best estimations are reached through calculations based on hypotheses about their shape and composition (Crifo 1988). Two extreme temperatures for each grain

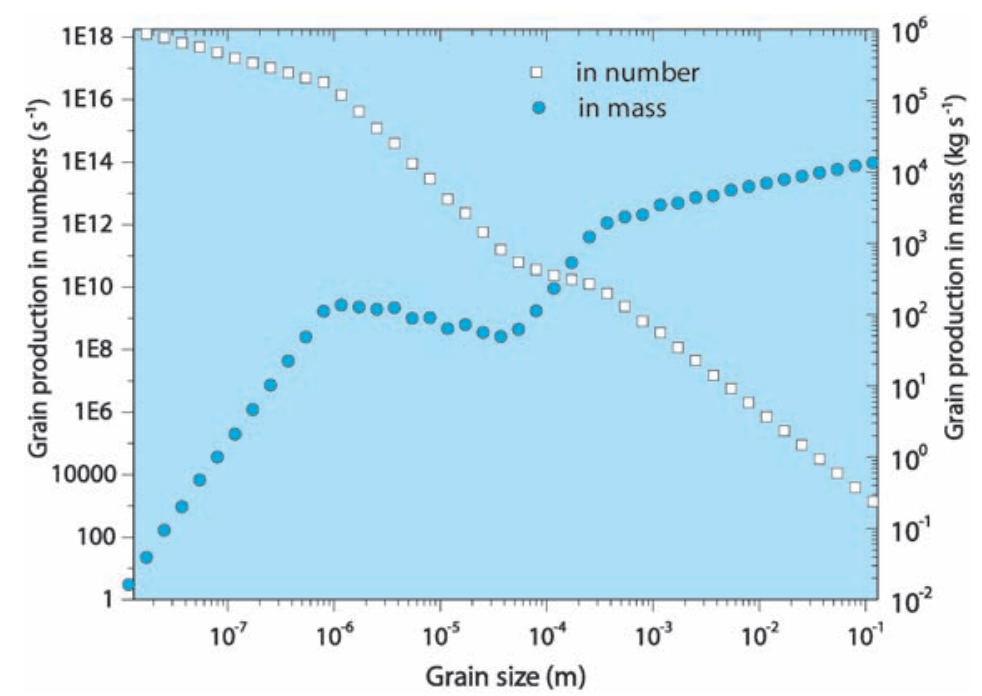

Fig. 8.6. Dust-grain production from comet Halley nucleus assuming a density of $1 \mathrm{~g} \mathrm{~cm}^{-3}$ (Cottin et al., 2004) 
size are achieved, depending on whether the grain is made of olivine (low temperature) or amorphous carbon (high temperature). Olivine represents the silicate nucleus of grains with a very low absorbance in the visible; it is a lower temperature limit for grains. Amorphous carbon $(\mathrm{aC})$ is representative of dark material, and stands as an upper limit for a strongly absorbing organic component of grains. As grain composition is poorly known, one cannot pretend to better constrain their temperature than between those two extremes. Grains temperatures are presented on Fig. 8.7 for a spherical shape; these temperatures are reached within the first few tens of kilometres from the nucleus (Crifo 1991). For porous or nonspherical grains the temperature tends to be higher.

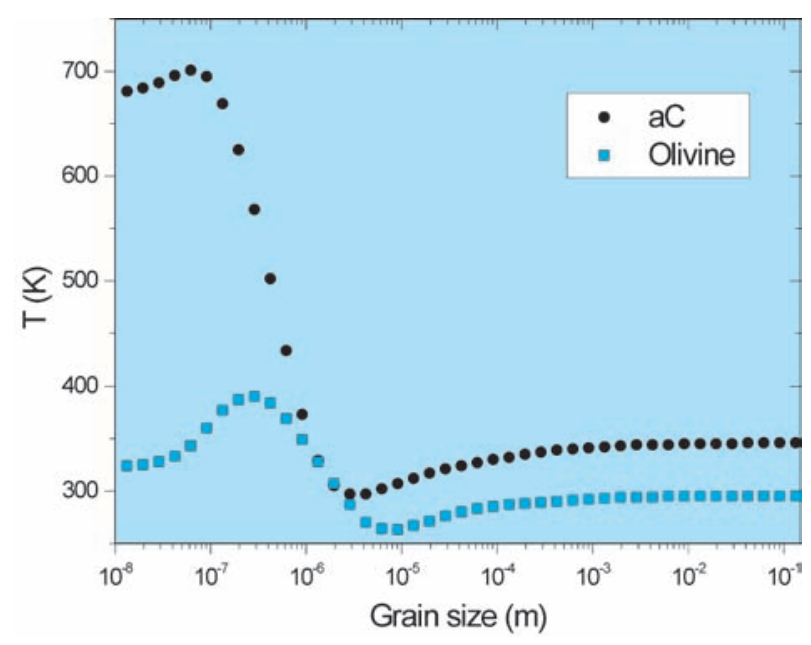

Fig. 8.7. Grain temperature as a function of size and composition (Cottin et al., 2004)

\subsubsection{Organics in Grains}

The most fruitful works related to the organic composition of grains have been reported in Kissel and Krueger 1987, and Krueger and Kissel 1987). To interpret the PUMA's mass spectra, they consider that all sudden dissipation processes near solid surfaces after an impact are governed by the same rules of molecular ion formation (Fig. 8.8). Such impacts occur when a dust particle hits the mass spectrometer's target. This hypothesis yields a good agreement between their predictions and other observations for small molecules (e.g. $\mathrm{HCN}, \mathrm{CH}_{3} \mathrm{CN}$ ).

For larger molecules the problem is more complex, as they cannot survive the impact process without fragmentation. In addition to the difficulty of assembling correctly the fragments to reconstitute the original molecule, the nature of the fragments themselves is uncertain, several fragments having the same mass (to the resolution of the spectrometer). Thus the identification by these authors 


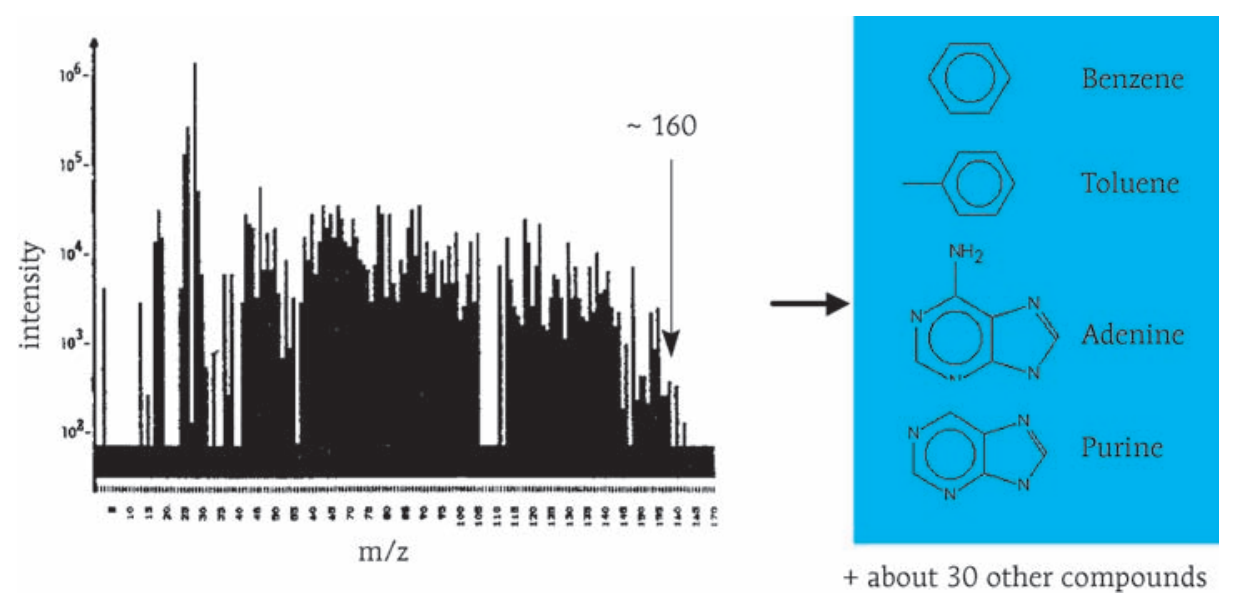

Fig. 8.8. Cumulated mass spectra collected in comet Halley by instrument PUMA on board VEGA-1 spacecraft and tentative interpretation. Adapted from Kissel and Krueger (1987)

of purines and adenine, for example, should be considered as very tentative. However, what is clearly shown by PUMA mass spectra is that compounds with complex structure and large molecular masses (at least with $\mathrm{m} / z$ up to $160 \mathrm{~g} \mathrm{~mol}^{-1}$ ) are present on grains.

First interpretations of the heavy-ion mass spectra from the PICCA instrument led Huebner (1987) to conclude the presence of polyoxymethylene (POM) in comet Halley. After further analyses, Huebner et al., (1989) discussed the presence of POM derivatives such as sulfur copolymers. As already stated in this chapter, the presence of POM in comets is coherent with the detection of extended sources of $\mathrm{H}_{2} \mathrm{CO}$ in the coma. Indeed, this molecule could be the degradation product of POM. The authors also suggest the presence of HCN polymers such as polyaminocyanomethylene (PACM: $\left.\left(\mathrm{NH}_{2}-\mathrm{C}-\mathrm{CN}\right)_{n}\right)$, even though these polymers are very difficult to detect because in the coma $\mathrm{HCN} / \mathrm{H}_{2} \mathrm{CO} \sim 0.1$ and because from thermodynamic considerations $\mathrm{N}$-containing polymers should be less volatile than $\mathrm{O}$ containing polymers (Krueger et al., 1991). The presence of $\mathrm{HCN} / \mathrm{H}_{2} \mathrm{CO}$ copolymers could also be suggested on comets because these two molecules polymerize very easily by themselves.

Later, however, Mitchell et al., (1992) showed that the mass spectrum pattern that led Huebner to announce the detection of polyoxymethylene is only characteristic of a mixing of molecules composed of $\mathrm{C}, \mathrm{H}, \mathrm{O}$ and $\mathrm{N}$ atoms (CHON molecules). Therefore PICCA mass spectra do not imply the presence of POM, but only of a large diversity of CHON molecules, among which POM could be present. The most we can say, if we consider laboratory simulation results presented latter in this chapter and the detection of an extended source of formaldehyde, is that the presence of POM on cometary nuclei is possible. 
Beside polymers, heteropolymers and large CHON molecules, other compounds have been suggested: pure carbon grains, PAHs (polycyclic aromatic hydrocarbons, i.e. aromatic cycles linked together), very "branched" aliphatic hydrocarbons (Fomenkova 1999). PAH detection from in situ UV spectroscopy in Halley was announced (Moreels et al., 1994); PAHs were not seen in the ISO spectra of Hale-Bopp, possibly due to the lower temperature of the comet at the time of these observations (Crovisier 1999). A weak residual feature at $3.28 \mu \mathrm{m}$ was, however, seen by Bockelée-Morvan et al., (1995) in various comets, and especially the dustier ones, which might be due to PAHs; this would correspond to a relative abundance to water 1.5 to $10 \times 10^{-6}$, at least 100 times below that deduced from UV data. A list of compounds probably and possibly detected in comet Halley grains by mass spectroscopy is reported in Table 8.2.

Table 8.2. Organic molecules inferred from mass spectra of gas and dust particles in comet Halley. The confidence levels of detections by MS are established as follows: Confirmed: Molecule also detected by remote observations; - High: Molecule not detected by remote observations but present after laboratory irradiations of cometary ice analogs; - Medium: Molecule detected only by mass spectroscopy with a good confidence level according to the authors; Low: Molecule only inferred by mass spectroscopy with a low confidence level according to the authors

\begin{tabular}{lllll}
\hline Molecule & Family & $\begin{array}{l}\text { Mass } \\
\text { spectrometer }\end{array}$ & $\begin{array}{l}\text { Confidence } \\
\text { level }\end{array}$ & Ref. \\
\hline $\begin{array}{l}\text { Hydrocyanic Acid } \\
\text { Methyl cyanide }\end{array}$ & C-N-H & PUMA, PICCA & Confirmed & $1,2,3$ \\
Acetonitrile & C-N-H & IMS & Confirmed & 7 \\
Methanol & C-O-H & NMS & Confirmed & $1,2,3$ \\
Formaldehyde & C-O-H & PUMA, PICCA & Confirmed & 3 \\
Formic acid & C-O-H & PUMA & Confirmed & 1,2 \\
Acetaldehyde & C-O-H & PUMA, PICCA & Confirmed & 1,3 \\
Ammonia & N-H & PUMA & Confirmed & 2 \\
Isocyanic acid & C-N-O-H & PUMA & Confirmed & 1,2 \\
Ethane & $\mathrm{C}-\mathrm{H}$ & NMS & Confirmed & 8 \\
Acetylene & $\mathrm{C}-\mathrm{H}$ & NMS & Confirmed & 8 \\
Acetic acid & $\mathrm{C}-\mathrm{O}-\mathrm{H}$ & PUMA & High & 1 \\
Polyoxymethylene & $\mathrm{C}-\mathrm{O}-\mathrm{H}$ & PICCA & High (itself or & 4,5 \\
& & & derivatives) & \\
Ethene & $\mathrm{C}-\mathrm{H}$ & NMS & High & 8 \\
Iminoethane & $\mathrm{C}-\mathrm{N}-\mathrm{H}$ & PUMA & Medium & 1,2 \\
Aminoethene & $\mathrm{C}-\mathrm{N}-\mathrm{H}$ & PUMA & Medium & 1,2 \\
Pyrroline & $\mathrm{C}-\mathrm{N}-\mathrm{H}$ & PUMA & Medium & 1 \\
\hline & & & & \\
\hline
\end{tabular}


Table 8.2. (continued)

\begin{tabular}{|c|c|c|c|c|}
\hline Molecule & Family & $\begin{array}{l}\text { Mass } \\
\text { spectrometer }\end{array}$ & $\begin{array}{l}\text { Confidence } \\
\text { level }\end{array}$ & Ref. \\
\hline Pyrrole & $\mathrm{C}-\mathrm{N}-\mathrm{H}$ & PUMA & Medium & 1,2 \\
\hline Imidazole & $\mathrm{C}-\mathrm{N}-\mathrm{H}$ & PUMA & Medium & 1 \\
\hline Pyridine & $\mathrm{C}-\mathrm{N}-\mathrm{H}$ & PUMA & Medium & 1,2 \\
\hline Pyrimidine & $\mathrm{C}-\mathrm{N}-\mathrm{H}$ & PUMA & Medium & 1,2 \\
\hline Ethyl cyanide & $\mathrm{C}-\mathrm{N}-\mathrm{H}$ & IMS & Medium & 7 \\
\hline Pentyne & $\mathrm{C}-\mathrm{H}$ & PUMA & Low & 1 \\
\hline Hexyne & $\mathrm{C}-\mathrm{H}$ & PUMA & Low & 1 \\
\hline Butadiene & $\mathrm{C}-\mathrm{H}$ & PUMA & Low & 1 \\
\hline Pentadiene & $\mathrm{C}-\mathrm{H}$ & PUMA & Low & 1 \\
\hline Cyclopentene & $\mathrm{C}-\mathrm{H}$ & PUMA & Low & 1 \\
\hline Cyclopentadiene & $\mathrm{C}-\mathrm{H}$ & PUMA & Low & 1 \\
\hline Clyclohexene & $\mathrm{C}-\mathrm{H}$ & PUMA & Low & 1 \\
\hline Cyclohexadiene & $\mathrm{C}-\mathrm{H}$ & PUMA & Low & 1 \\
\hline Benzene & $\mathrm{C}-\mathrm{H}$ & PUMA & Low & 1 \\
\hline Toluene & $\mathrm{C}-\mathrm{H}$ & PUMA & Low & 1 \\
\hline Propanenitrile & $\mathrm{C}-\mathrm{N}-\mathrm{H}$ & PUMA & Low & 1 \\
\hline Iminomethane & $\mathrm{C}-\mathrm{N}-\mathrm{H}$ & PUMA & Low & 1 \\
\hline Iminopropene & $\mathrm{C}-\mathrm{N}-\mathrm{H}$ & PUMA & Low & 1 \\
\hline Purine & $\mathrm{C}-\mathrm{N}-\mathrm{H}$ & PUMA & Low & 1,2 \\
\hline Adenine & $\mathrm{C}-\mathrm{N}-\mathrm{H}$ & PUMA & Low & 1,2 \\
\hline Polyaminocyanomethylene & $\mathrm{C}-\mathrm{N}-\mathrm{H}$ & PICCA & Low & 5 \\
\hline Methanolnitrile & $\mathrm{C}-\mathrm{N}-\mathrm{O}-\mathrm{H}$ & PUMA & Low & 1 \\
\hline Methanalimine & $\mathrm{C}-\mathrm{N}-\mathrm{O}-\mathrm{H}$ & PUMA & Low & 1 \\
\hline Aminomethanol & $\mathrm{C}-\mathrm{N}-\mathrm{O}-\mathrm{H}$ & PUMA & Low & 2 \\
\hline Aminomethanal & $\mathrm{C}-\mathrm{N}-\mathrm{O}-\mathrm{H}$ & PUMA & Low & 2 \\
\hline Oxyimidazole & $\mathrm{C}-\mathrm{N}-\mathrm{O}-\mathrm{H}$ & PUMA & Low & 1 \\
\hline Oxypyrimidine & $\mathrm{C}-\mathrm{N}-\mathrm{O}-\mathrm{H}$ & PUMA & Low & 1 \\
\hline Xanthine & $\mathrm{C}-\mathrm{N}-\mathrm{O}-\mathrm{H}$ & PUMA & Low & 1 \\
\hline
\end{tabular}

1 (Kissel and Krueger 1987); 2 (Krueger and Kissel 1987); 3 (Krueger et al., 1991); 4 (Huebner 1987); 5 (Huebner et al., 1989); 6 (Eberhardt and Krankowsky 1995); 7 (Geiss et al., 1999); 8 (Altwegg et al., 1999)

\subsubsection{Cometary Silicates}

The nature of other refractory components is constrained by infrared observations of comets. Identification is achieved through a comparison of observations with laboratory spectra of different kinds of silicates and possibly carbonaceous 
grains, and it should be noted that there is no unique solution to the fitting problem. It appears that grains are made of a mixture of crystalline and amorphous silicate (Brucato et al., 1999, Crovisier et al., 1997, Harker et al., 2002, Hayward and Hanner 1997, Nuth et al., 2002, Wooden et al., 2000).

Crystalline forsterite (a magnesium-rich olivine) has been detected thanks to the ISO infrared satellite (Crovisier et al., 1997). Ground-based telescopes have enabled a study of the infrared spectrum around $10 \mu \mathrm{m}$, and determined the ratio pyroxene/olivine. According to Harker et al., (2002) amorphous silicates make up about $70 \%$ (by mass) of the submicrometre-sized $(\leq 1 \mu \mathrm{m})$ silicate dust grains in Hale-Bopp's coma, and are distributed roughly equally between olivine and pyroxene composition, and between Fe-rich and Mg-rich species; the remaining $30 \%$ are predominantly Mg-rich crystalline silicates with a crystalline
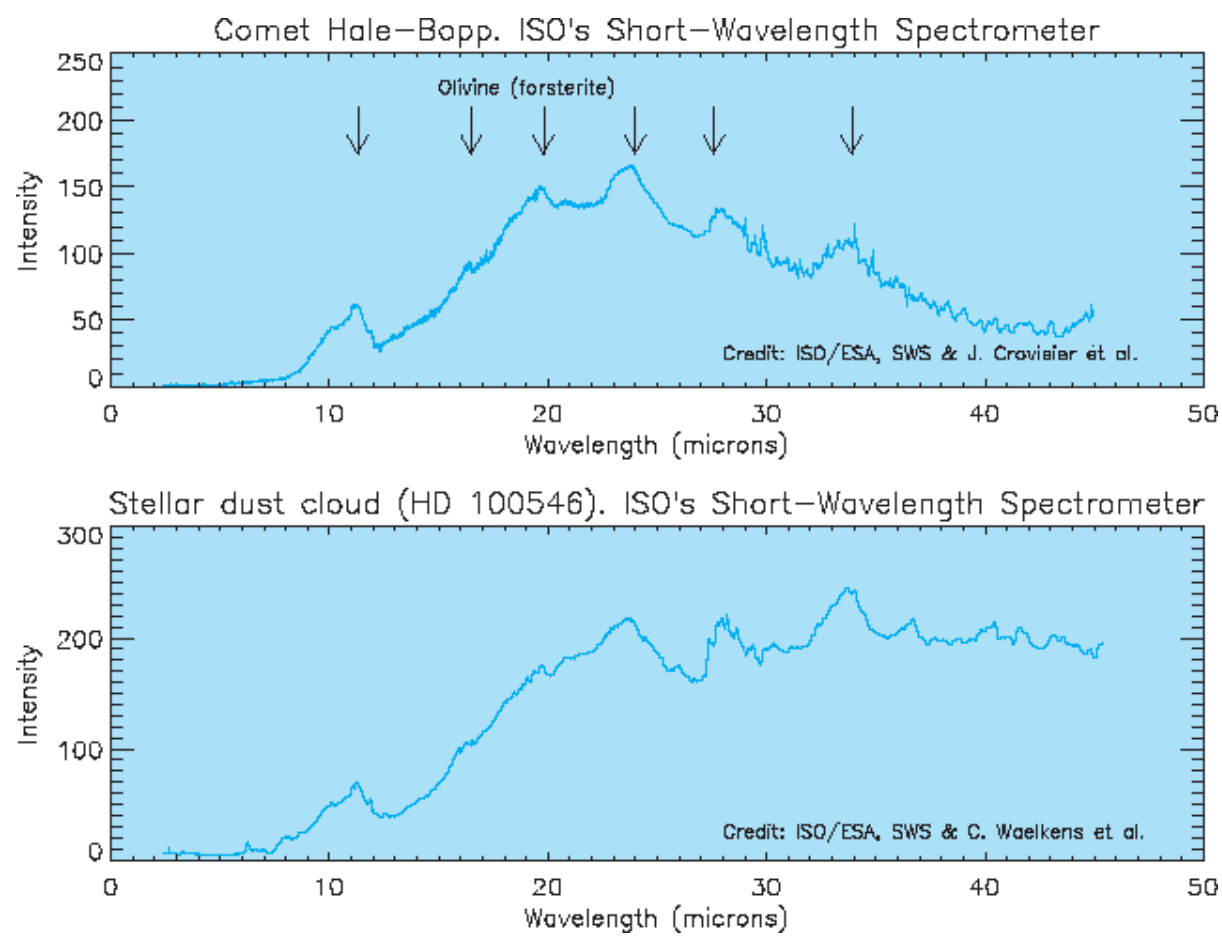

Fig. 8.9. Comparison of the infrared spectrum of Hale-Bopp obtained by ESA's Infrared Space Observatory ISO (Crovisier et al., 1997) with that of a young star of type AeBe (e.g. Malfait et al., 1998). The features in the comet spectrum lead to the identification of a crystalline silicate, fosterite, a magnesium-rich olivine. The AeBe stars are slightly more massive than the Sun, but the dust that surrounds them and that is responsible for this IR emission is considered to be similar to dust in the protosolar nebula. In HD100456, the similarity is even more striking and points towards a high crystalline mass fraction and small particle size $(<10 \mu \mathrm{m})$ in both cases (Bouwman et al., 2003) 
olivine:orthopyroxene ratio about 4 to $5: 1$ that seems to vary slightly with heliocentric distance. Figure 8.9 shows a comparison between the infrared spectrum of Hale-Bopp and circumstellar matter around a young star. However, data of such a high quality is only available for one comet (Hale-Bopp).

\subsubsection{Elemental and Isotopic Composition}

\subsubsection{Elements}

The composition in elements that can be deduced by combining dust and gas measurements (Geiss 1988) leads to abundances normalized to silicon close to solar abundances for carbon and oxygen. However, one notes a depletion (underabundance) of hydrogen of about 600 , due to the high $\mathrm{H}_{2}$ volatility and a depletion of 2 for nitrogen, which is explained in the same way by the fact that nitrogen is often in the form of $\mathrm{N}_{2}$, a very volatile gas, too (Fig. 8.10). The

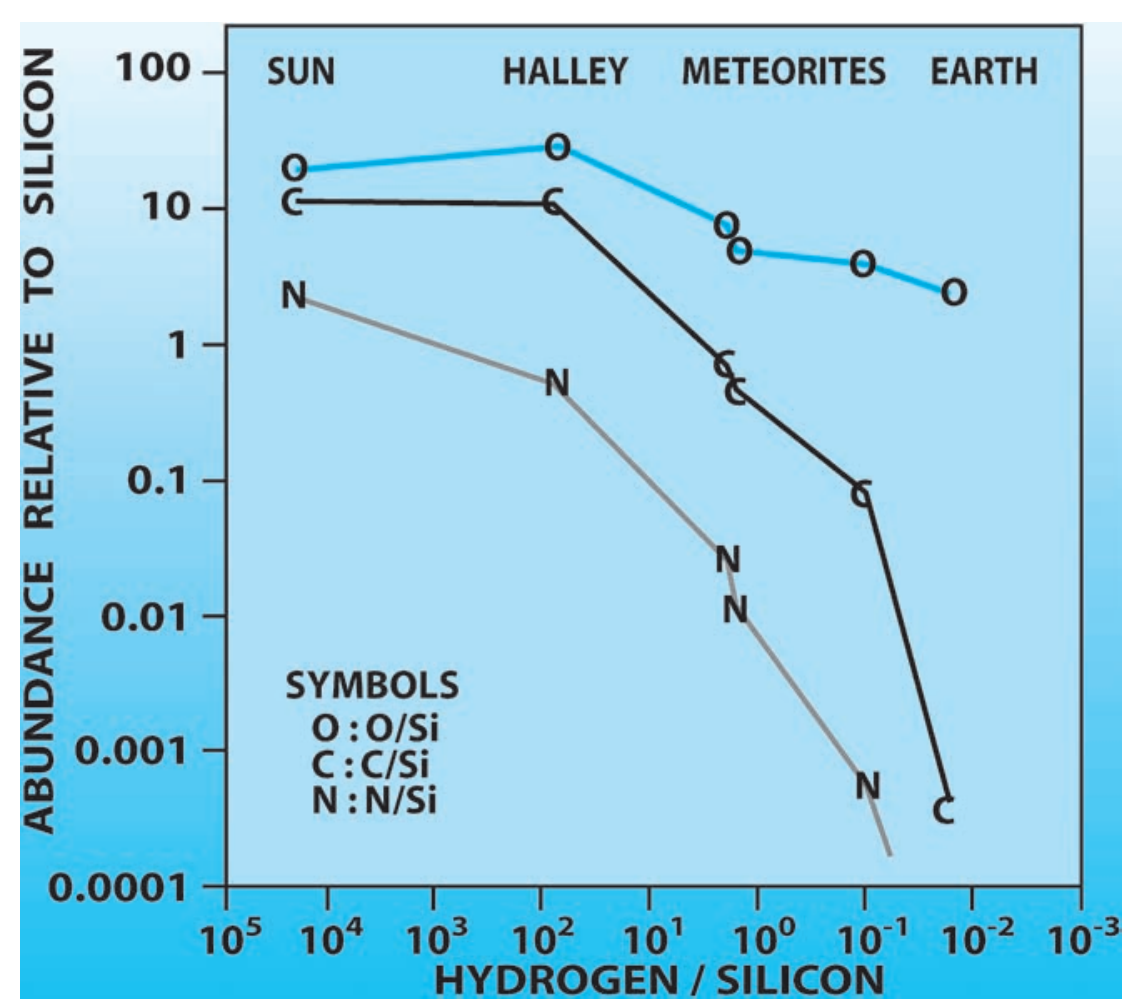

Fig. 8.10. Comparison of elemental abundances in the Sun, comet Halley, and (C1, C2) meteorites (Geiss 1988). The element abundance ratios in $\mathrm{P} /$ Halley are uncertain by a factor of about two (excluding the variability and uncertainty in the gas/dust ratio) 
gas:dust ratio derived in the case of Halley is 1:2 in mass (Jessberger and Kissel 1991); note that the computation of the elemental composition for the whole comet uses this ratio and is quite sensitive to it.

In Fig. 8.10, the elemental composition of comets is also compared with that of carbonaceous chondrites (Geiss 1988). Elements of the series $\mathrm{Mg}-\mathrm{Al}-$ $\mathrm{Ca}$, as well as oxygen have comparable abundances within a factor of 2 . On the other hand, in comet Halley, abundances of carbon, hydrogen, nitrogen and of any other element appearing mostly in volatile compounds are larger than in carbonaceous chondrites. This leads us to say that cometary matter is more "pristine" than that of carbonaceous chondrites, in the sense that it underwent less heating and thus was less processed.

\subsubsection{Isotopic Abundances}

Much of the information on the origin of cometary matter and on the importance of the cometary contribution to the Earth's water content derives from the study of isotopic abundances, which have been recently reviewed by Altwegg and Bockelée-Morvan (2003). The $\mathrm{D} / \mathrm{H}$ ratio in particular is very important data to investigate the origin of cometary matter (e.g. Bockelée-Morvan et al., 1998, Mousis et al., 2000, Robert 2002) and its delivery to the Earth (Dauphas et al., 2000; see Sect. 8.5.3). It was measured for two species: $\mathrm{HDO} / \mathrm{H}_{2} \mathrm{O}$ $\left(\mathrm{D} / \mathrm{H} \sim 3 \times 10^{-4}\right)$ and $\mathrm{DCN} / \mathrm{HCN}\left(\mathrm{D} / \mathrm{H} \sim 2.3 \times 10^{-3}\right)$; upper limits on the order of $10^{-2}$ or below have also been set for deuterated formaldehyde, methanol, ammonia and $\mathrm{H}_{2} \mathrm{~S}$. Molecules appear enriched in deuterium: as can be seen in Fig. 8.11, D/H in water for the 3 comets for which it has been measured is twice the ocean SMOW value, and an order of magnitude higher than the protosolar value of $2.5 \times 10^{-5}$. This enrichment is however not as high as in the interstellar gas (where multiply deuterated species like $\mathrm{D}_{2} \mathrm{CO}$ and even $\mathrm{ND}_{3}$ have been observed). Measurements of the $\mathrm{O}, \mathrm{C}, \mathrm{N}$ and $\mathrm{S}$ isotopes display, in general, abundances very close to solar abundances (e.g. Jewitt et al., 1997, Lecacheux et al., 2003), which confirms that the comets are Solar System objects. This does not contradict the assumption of an interstellar origin of the cometary matter discussed below. Indeed, the Sun and the Solar System as a whole were formed from a homogeneous clump of interstellar matter and it is from the same fragment of IS matter that the comets were made.

Some carbonaceous grains analyzed by in situ mass spectroscopy in Halley (Jessberger and Kissel 1991) showed a very high ratio ${ }^{12} \mathrm{C} /{ }^{13} \mathrm{C}$, rising up to 5000 , whereas the normal ratio in Solar System objects is 89 . These small grains would have been formed in the envelope of certain stars known as AGB stars (asymptotic giant branch) and would then have been injected into the interstellar medium. They thus would have been mixed with the interstellar cloud fragment that condensed to form the protosolar nebula, then later incorporated as such in the matter of forming comets. As this high departure from solar ${ }^{12} \mathrm{C} /{ }^{13} \mathrm{C}$ values is concentrated in very small grains, which do not represent a large quantity 


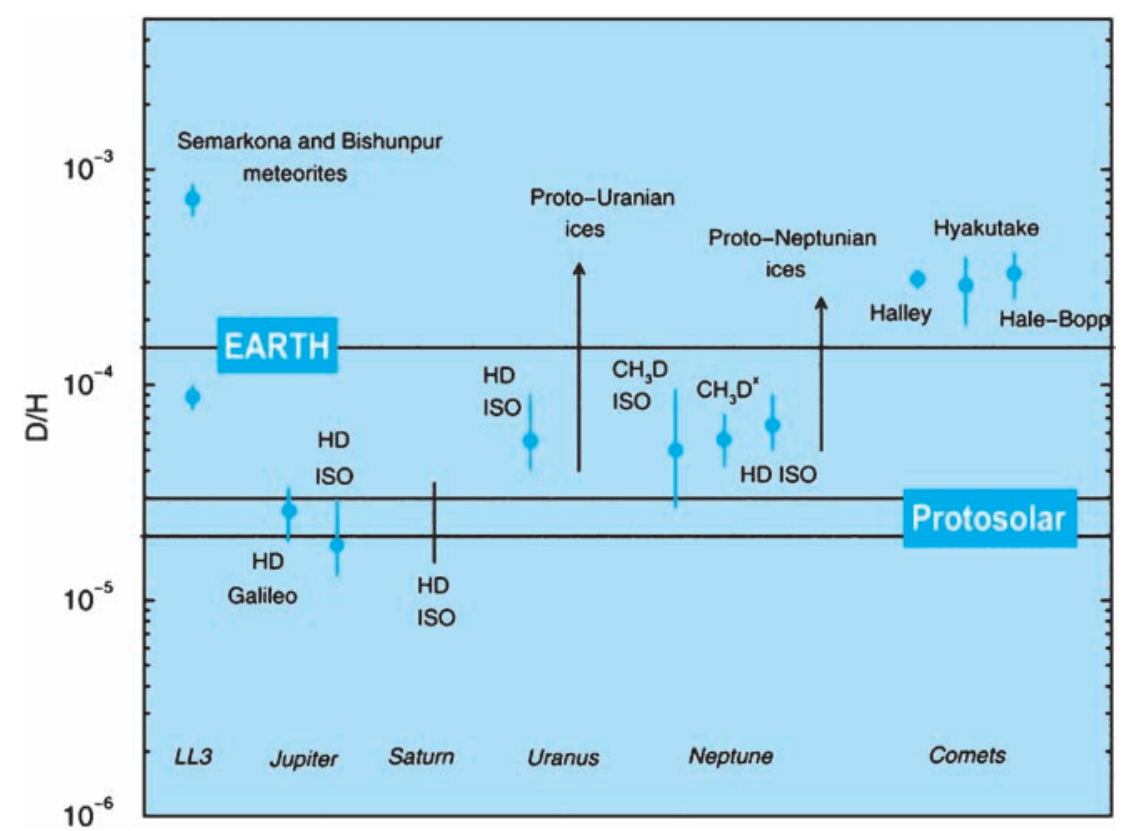

Fig. 8.11. D/H ratios in the Solar System. For comets, only measurements of D/H in water are shown (from Hersant et al., 2001)

of carbon, the average value of the cometary ${ }^{12} \mathrm{C} /{ }^{13} \mathrm{C}$ ratio remains close to the solar value, namely approximately 89. An intriguing measurement has been made in CN (Arpigny et al., 2003) suggesting the existence of parents of the $\mathrm{CN}$ radical other than $\mathrm{HCN}$ - possibly solid organic compounds - with strongly nonsolar $\mathrm{N}$ isotopic ratios.

\subsubsection{Are All Comets Similar?}

Abundance variations are observed from one comet to another, in particular for $\mathrm{CO}$ (from less than $1 \%$ to more than $30 \%$ according to comets and to the distance from the Sun) and $\mathrm{CH}_{3} \mathrm{OH}$ (1 to 5\%; e.g. Bockelée-Morvan 1997). This composition is deduced from gas abundances measured in the coma, making assumptions on the spatial distribution of the molecules, the limited chemistry in the coma, and the sublimation process.

That all comets are not similar already has observational evidence, as seen from dust content and daughter species $\mathrm{C}_{2}, \mathrm{CN}$ (A'Hearn et al., 1995, Rolfe and Battrick 1987). Column 3 in Table 8.1 shows the range spanned by the observed molecular abundances in a sample of comets (Biver et al., 2002). Different compositions are expected, to some extent, between comets coming from the Oort cloud and the Kuiper Belt due to their different formation zone (respectively Jupiter-Neptune versus mostly transneptunian, see Sect. 8.4 and Morbidelli and 
Table 8.3. Molecules detected during experimental simulations of cometary ice analogs. Italic letters refer to molecules actually detected in comets. (t) means tentative detection only in the analogs. Amino acids (alanine, AIB, ... except glycine) were detected after acid hydrolysis of the room temperature residue. Updated from Cottin et al., 1999

\begin{tabular}{|c|c|c|}
\hline Hydrocarbons: & Ketones: & Alcotiols: \\
\hline $\mathrm{CH}_{4}$ & $\mathrm{CH}_{3} \mathrm{COCH}_{3}$ & $\mathrm{CH}_{3} \mathrm{OH}$ \\
\hline $\mathrm{C}_{2} \mathrm{H}_{2}, \mathrm{C}_{2} \mathcal{H}_{4}, \mathrm{C}_{2} \mathrm{H}_{6}$ & $\mathcal{H O C H}_{2} \mathrm{COCH}_{3}$ & $\mathrm{CH}_{3} \mathrm{CH}_{2} \mathrm{OH}$ \\
\hline $\mathcal{C}_{3} \mathcal{H}_{8}, \mathcal{C}_{4} \mathcal{H}_{10}$ & $\mathcal{H O C H}_{2} \mathrm{CH}_{2} \mathrm{COC} \mathcal{H}_{3}$ & $\mathcal{H O C H}_{2} \mathrm{CH}_{2} \mathrm{O} \mathcal{H}$ \\
\hline$C_{5} \mathcal{H}_{10}, \mathcal{C}_{5} \mathcal{H}_{12}$ & & $\mathcal{H O C H}_{2} \mathrm{CH}(\mathrm{OH}) \mathrm{CH}_{6} \mathrm{O} \mathcal{H}$ \\
\hline $\mathcal{C}_{6} \mathcal{H}_{12}, \mathcal{C}_{6} \mathcal{H}_{14}$ & & $\mathrm{C}_{4} \mathcal{H}_{8}(O \mathcal{H})_{2}$ \\
\hline $\mathcal{C}_{7} \mathcal{H}_{16}$ & & $\mathrm{C}_{5} \mathcal{H}_{9} O \mathcal{H}(t)$ \\
\hline Amides: & $\begin{array}{l}\text { Carboxylic acids: } \\
\mathrm{HCOOH}\end{array}$ & $\mathrm{C}_{5} \mathcal{H}_{11} \mathrm{OH}$ \\
\hline $\mathrm{NH}_{2} \mathrm{CHO}$ & $\mathrm{CH}_{3} \operatorname{COO} \mathcal{H}(t)$ & Amino Acids: \\
\hline $\mathrm{CH}_{3} \mathrm{CON} \mathcal{H}_{2}$ & $\mathcal{H O C H}_{2} \mathrm{COOH}$ & $\mathcal{N} \mathcal{H}_{2} \mathrm{CH}_{2} \operatorname{COOH} \mathcal{H}(G$ lycine $)$ \\
\hline $\mathcal{H O C H}_{2} \mathrm{CON} \mathcal{H}_{2}$ & $\mathcal{H O C H} \mathcal{H}_{2} \mathcal{H}(O \mathcal{H}) \mathcal{C O O H}$ & $\mathcal{N} \mathcal{H}_{2} \mathcal{C H}\left(\mathcal{C H}_{3}\right)$ COO H $($ Alanine) \\
\hline $\mathcal{N} \mathcal{H}_{2}(\mathrm{CO})_{2} \mathcal{N}_{\mathcal{H}}$ & $\mathcal{H O C H}_{2} \mathrm{CH}_{2} \mathrm{COOH}$ & $\mathrm{CH}_{3} \mathcal{C H}_{2} \mathcal{C H}\left(\mathfrak{N} \mathcal{H}_{2}\right) \mathcal{C O O H} \quad(\alpha \mathcal{A B A})$ \\
\hline $\mathcal{H O C H}_{2} \mathrm{CH}(\mathrm{O} \mathcal{H}) \mathrm{CON} \mathcal{H}_{2}$ & $\mathcal{N} \mathcal{H}_{2} \mathrm{COCOOH}$ & $\begin{array}{l}\mathcal{C H}_{3} \mathcal{C H}\left(\mathcal{N} \mathcal{H}_{2}\right) \mathcal{C H} \mathcal{H}_{2} \operatorname{COOH}(\beta \mathcal{A B A}) \\
\left(\mathcal{C H}_{2} \mathcal{N}\left(\mathcal{H}_{2}\right)\left(\mathcal{C H}_{3}\right) \mathcal{C H C O O H}(\mathcal{A} I \mathcal{H})\right.\end{array}$ \\
\hline & Aromatic Co & Sarcosine \\
\hline Amines: & & Ethylglycine \\
\hline $\mathcal{H O C H}_{2} \mathrm{CH}_{2} \mathfrak{N H}_{2}$ & & Valine, Proline, Serine \\
\hline $\mathcal{H C N} \mathcal{H}\left(\mathcal{N}\left(\mathcal{H}_{2}\right)\right.$ & & Aspartic acid \\
\hline Diaminopyrrole & & Diaminopropanoic acid \\
\hline Diaminofurane & & Diaminobutyric acid \\
\hline $\begin{array}{l}\text { Triaminopropane } \\
\left(\mathcal{C H}_{2}\right)_{6} \mathcal{N}_{4}(\mathcal{H} \mathcal{M} \mathcal{T})\end{array}$ & & $\begin{array}{l}\text { Diaminopentanoic acid } \\
\text { Diaminofe xanoic acid }\end{array}$ \\
\hline Aldefiydes: & Ethers: & Esters: \\
\hline $\mathrm{H}_{2} \mathrm{CO}$ & $\mathrm{CH}_{3} \mathrm{OCH}_{2} \mathrm{OCH}_{3}(t)$ & $\mathrm{HCOOCH}_{3}$ \\
\hline $\mathrm{CH}_{3} \mathrm{OCH}_{2} \mathrm{CHO}$ & $\mathrm{C}_{3} \mathcal{H}_{6} \mathrm{O}_{3} \quad(\mathcal{T}$ rioxane $)(t)$ & $\mathrm{CH}_{3} \mathrm{COOCH}_{3}$ \\
\hline & $\left(-C \mathcal{H}_{2}-O-\right)_{n}(\mathcal{P O} \mathcal{M})$ & $\mathrm{CH}_{3} \mathrm{CH}_{2} \mathrm{COOCH} \mathcal{H}_{3}$ \\
\hline
\end{tabular}

Brown 2004). Observational evidence has been claimed for variations in the $\mathrm{C}_{2} \mathrm{H}_{6} / \mathrm{H}_{2} \mathrm{O}$ and $\mathrm{CH}_{4} / \mathrm{H}_{2} \mathrm{O}$ ratios (Mumma et al., 2002, and Gibb et al., 2003, respectively), but the statistics is still limited.

\subsection{Laboratory Simulation of Cometary Matter}

\subsubsection{Experimental Simulations}

Only volatile molecules have been detected so far in cometary atmospheres, as no direct cometary sample has ever been analyzed (except for IDPs, whose origin cannot, however, be uniquely assigned to comets). For a better insight into more complex, less volatile material, one has to turn to experimental laboratory work. The principle of such experiments is the following: from observations of the most abundant species in comas and in the interstellar medium, one can infer the 
probable composition of the nucleus ices. A gaseous sample of the key species is deposited under vacuum on a cold substrate and irradiated during or after deposition by UV photons or charged particles. Condensed ices are sometimes simply warmed up slowly without irradiation. When the sample is warmed up for analysis a refractory organic residue remains on the substrate as the volatiles sublimate (Fig. 8.12). Greenberg has called this residue "yellow stuff". Bernstein et al., (1997) have shown that the organic residue is formed only when the initial mixture of ices contains polar molecules such as $\mathrm{CH}_{3} \mathrm{OH}$ and $\mathrm{NH}_{3}$.

The diversity of organic compounds synthesized is remarkable but their identification is seldom exhaustive. Table 8.3 is a simplified list of all the detected compounds. The simplest compounds such as $\mathrm{CO}, \mathrm{CO}_{2}, \mathrm{H}_{2} \mathrm{CO}$ and $\mathrm{CH}_{4}$ are

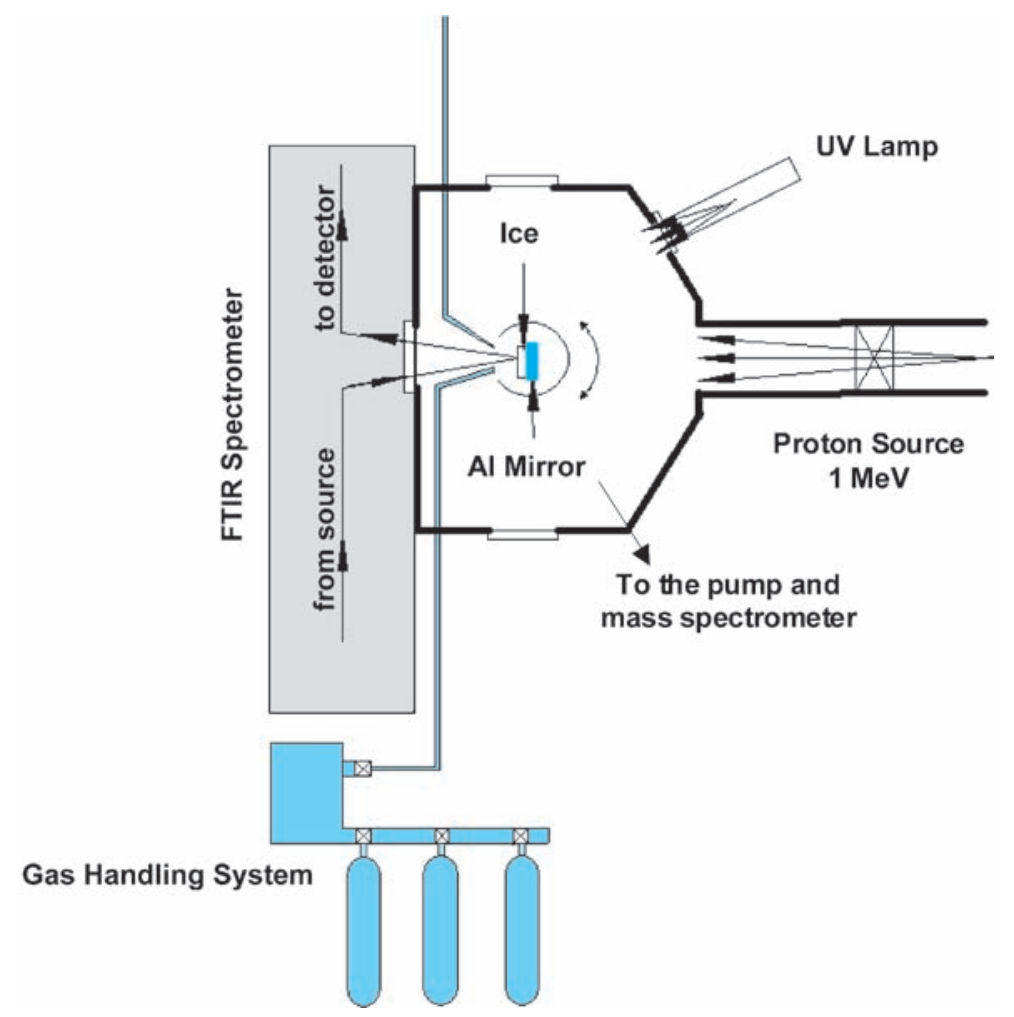

Fig. 8.12. A typical experimental setup allowing the irradiation (by UV and/or energetic protons) of cometary ice analogs made by deposition of a gas mixture on a rotating aluminium mirror cooled down to $10 \mathrm{~K}$ in a cryostat. The ice evolution can be analysed in situ by infrared reflection spectroscopy, and the volatiles released during warming, by mass spectrometry. The room-temperature residue can be collected for further analysis such as GC-MS (gas chromatography coupled to mass spectrometry), HPLC (high-performance liquid chromatography) and many others. From Hudson and Moore (1999) 
detected in almost all the experiments, if the irradiated ice contains the appropriate elements. For more complex molecules, it depends on the ice composition and the nature of the energy source.

In addition to chemical transformations, it must be mentioned that experiments on the trapping of gases during ice condensation suggest that these processes play an important role in determining the composition of the ice and could lead to important enrichment or depletion between the gaseous and solid phases (Notesco and Bar-Nun 1996, Notesco and Bar-Nun 1997, Notesco et al., 1997).

\subsubsection{Energy Deposition}

Three kinds of energetic processing occur on icy coated dust grains in interstellar clouds (potentially precometary ices - see Sect. 8.4) or in the outer layers of comet ices in the Solar System:

In interstellar clouds, icy coated dust particles are subjected to processing by:

- Charged particles: Galactic cosmic rays.

- UV-photons: Lyman $\alpha$ photons from neighboring stars (in the diffuse outer regions of a cloud), or UV-photons induced by galactic cosmic rays (in the inner regions of dense clouds).

- Thermal processes: Cycling between the cold centre of a dense cloud and its warmer diffuse outer regions.

In the Solar System, the outer layers of comets undergo the same processes:

- Charged particles: Galactic cosmic rays, mainly in the Kuiper belt and the Oort cloud. This process has the largest effect on the outer few metres of the nucleus.

- UV-photons: Solar-UV, mainly in the inner Solar System when the comet is close to the Sun. This process would affect the outer few micrometres of the nucleus. Also during the Solar System formation, in the external layers of the disk, when solar UV luminosity was much higher than today.

- Thermal processes: During the formation of the Solar System (depending on the region in which the comet accretes), and in the inner Solar System (when the comet approaches perihelion).

Due to the diversity of environments involved, constraining the degrees to which different processes affect cosmic ices is a highly convoluted problem. Differences between the products synthesized during processing, according to the energy sources, could give information on the history of cometary matter and comets. Investigations are in progress to address this question.

\subsubsection{UV Irradiation}

UV irradiation is performed using a flowing-hydrogen discharge lamp (powered by a microwave cavity) delivering mainly Lyman $\alpha$ photons $(122 \mathrm{~nm})$ and a broad 
band of photons centred at $160 \mathrm{~nm}$ (see Allamandola et al., 1988, and Cottin et al., 2003) for a detailed description). The irradiated ices comprise common cometary small molecules but the initial abundances of $\mathrm{CH}_{3} \mathrm{OH}, \mathrm{NH}_{3}$ and/or $\mathrm{CO}$ relative to $\mathrm{H}_{2} \mathrm{O}$ are usually higher than those deduced for present-day comets and displayed in Table 8.1. During these experiments a wide variety of organic compounds have been identified. From an initial mix of $\mathrm{H}_{2} \mathrm{O}: \mathrm{CO}: \mathrm{NH}_{3}$ (ratio $=5: 5: 1$ ), glycine, the simplest amino acid, acetamide, glyceramide, and many other molecules have been detected by GC-MS (Briggs et al., 1992). Analysis by MS-MS on the organic residues formed, leads to the detection of heavier compounds: several cyclic molecules and PAHs (Greenberg and Mendoza-Gomez 1993). The composition of the heaviest part of the residue is still unknown but an elemental composition based on the overall structure of the mass spectra is given by Greenberg and Li (1998) (C: O: $\mathrm{N}: \mathrm{H}=1: 0.06:>0.001: 1.1$ ).

Among the molecules synthesized after such irradiations of ices, one of them is of great interest. Bernstein et al., (1995) have identified abundant hexamethylenetetramine (HMT $-\mathrm{C}_{6} \mathrm{H}_{12} \mathrm{~N}_{4}$ ) in the refractory residue. This compound has exobiological implications since its acid hydrolysis products are amino acids (Wolman et al., 1971). Typically, for an initial composition of $\mathrm{H}_{2} \mathrm{O}: \mathrm{CH}_{3} \mathrm{OH}$ : $\mathrm{CO}: \mathrm{NH}_{3}$ (10: 5: 1: 1), the organic residue at $300 \mathrm{~K}$ contains $\mathrm{HMT}(\sim 60 \%)$, ethers and POM-like polymers $(\sim 20 \%)$, ketones and amides $(\sim 20 \%) \cdot 1 / 5$ of the carbon and $1 / 2$ of the nitrogen from the initial ice composition remain in the refractory part (Bernstein et al. 1995). Thus a large fraction of HMT is formed ( $60 \%$ of the residue) whereas only $5 \% \mathrm{NH}_{3}$ is present in the ice before irradiation. A scheme of HMT production is shown in Fig. 8.13. Formaldehyde is produced by methanol UV oxidation. It then reacts with ammonia to produce methylimine and its trimer: hexahydro-1,3,5-triazine. Successive reactions with formaldehyde and ammonia result in the formation of HMT (Bernstein et al., 1995). Methanol plays a key role and it has been shown by ${ }^{13} \mathrm{C}$ isotopic substitution that it is the source of HMT's carbon. The production of HMT and some HMT family molecules has been studied in great detail by Muñoz Caro (2003).

HMT photolysis produce nitriles ( $\mathrm{HCN}$ and $\mathrm{RCN}$ ) and isonitriles (RNC) (Bernstein et al 1994, Cottin et al 2002). Knowing this, it is interesting to note that it has been shown that the $\mathrm{CN}$ radical might have an extended source in comet Halley (Klavetter and A'Hearn 1994). According to these authors, this source could be large molecules present on grains because the observed HCN cannot explain the amount of $\mathrm{CN}$ in comets. Other observed $\mathrm{CN}$-containing compounds ( $\mathrm{HNC}, \mathrm{CH}_{3} \mathrm{CN}, \mathrm{HC}_{3} \mathrm{~N}$ ) are only minor products and cannot explain the discrepancy. Thus having been produced in the interstellar ices, as we have just seen, HMT could act as a parent $(\mathrm{HMT} \rightarrow \mathrm{CN})$ or "grandparent" (HMT $\rightarrow$ $\mathrm{RCN} \rightarrow \mathrm{CN}$ ) molecule for the $\mathrm{CN}$ extended source. As yet, there has been no observation of HMT in comets. If present, its detection in the IR would be masked by the $\mathrm{Si}-\mathrm{O}$ and $\mathrm{C}-\mathrm{O}$ vibration bands that are in the same region as the strongest HMT infrared signatures. Direct detection of HMT should be 


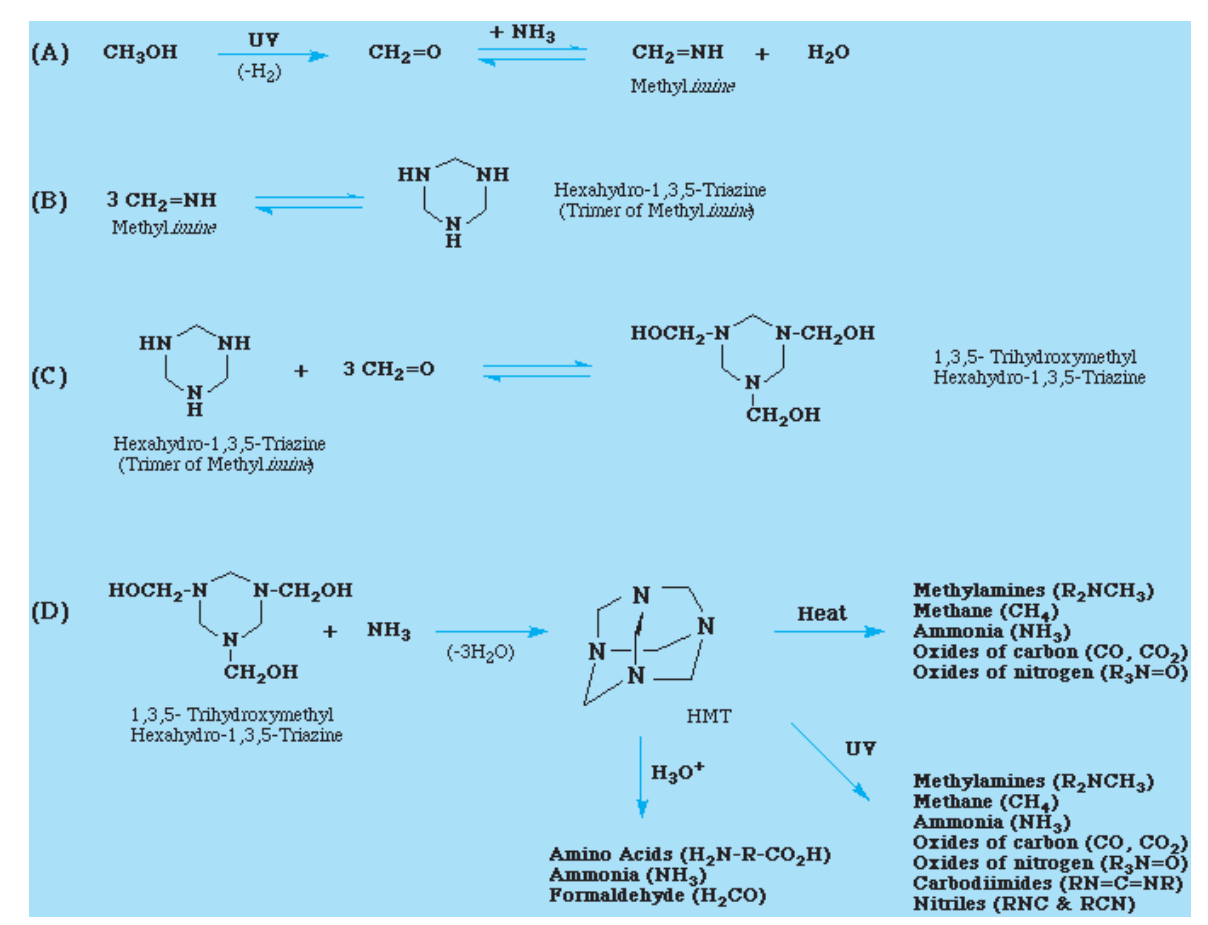

Fig. 8.13. Hexamethylenetetramine (HMT) chemistry. From (Bernstein et al., (1995))

feasible by in situ measurements with the GC-MS on board the lander of the Rosetta mission.

Bernstein et al (2002) and Muñoz Caro et al (2002) have detected a great number of amino acids (such as glycine, alanine, sarcosine, valine, proline, serine, etc.) in residues obtained after UV irradiation of ice mixtures made of $\mathrm{H}_{2} \mathrm{O}: \mathrm{NH}_{3}$ : $\mathrm{CH}_{3} \mathrm{OH}: \mathrm{HCN}$ and $\mathrm{H}_{2} \mathrm{O}: \mathrm{CH}_{3} \mathrm{OH}: \mathrm{NH}_{3}$ : $\mathrm{CO}: \mathrm{CO}_{2}$, respectively. Unhydrolyzed residues produce only a trace of glycine whose detection has already been reported by Briggs et al (1992) without any liquid water introduced to the analysis protocol. The detection of the other amino acids requires an acid hydrolysis of the residue in very strong conditions $\left(\mathrm{HCl} \geq 6 \mathrm{M}\right.$ and $\left.T \geq 100^{\circ} \mathrm{C}\right)$. Therefore it is not clear to date if 1) amino acids are present themselves in the laboratory residues, and henceforth in cometary ices, or if "only" amino acids precursors are synthesized, and 2) if the residues' processing (acid hydrolysis) is relevant to any chemistry that could have turned the amino acids' precursors imported by cometary impacts in the primitive oceans of the early Earth into actual amino acids. Note that the hydrolysis conditions would break a peptidic bond; some dipeptides have been found in the extraterrestrial organic matter of the Yamato791198 and Murchison meteorites, but only glycine-glycine dimers and with a low $\left(10^{-4}\right)$ abundance relative to glycine (Shimoyama and Ogasawara 2002). 


\subsubsection{Irradiation by Charged Particles}

Important work concerning the particle bombardment of ices has been performed by Strazzulla's team in Catania and in Marla Moore's laboratory at NASA Goddard Space Flight Center. The particles used are $\mathrm{H}^{+}, \mathrm{He}^{+}, \mathrm{N}^{+}$or Ar. The bombardment of a large diversity of carbon-containing ices induces an evolution toward an amorphous material called Strazzulla "ion-produced hydrogenated amorphous carbon" (IPHAC) (Strazzulla 1997; Strazzulla and Baratta 1991; Strazzulla et al 1991).

The general results are the following: up to a dose of about $10 \mathrm{eV} / \mathrm{C}$ atom the ice is partially converted into a refractory material. From 10 to $25 \mathrm{eV} / \mathrm{C}$ atom, a massive loss of $\mathrm{H}$ is observed and the target evolves to an organic material made of chemical chains of different sizes. For stronger irradiations $(\geq 25 \mathrm{eV} / \mathrm{C}$ atom) IPHAC, the ultimate state of organic degradation, is formed (Strazzulla 1997). It has been shown by Jenniskens et al. (1993) that energetic UV (10eV) irradiation of the organic residue of processed ices also leads to IPHAC formation, which then can be also called: "irradiation-produced hydrogenated amorphous carbon". Thus, after a typical lifetime in the interstellar medium, UV radiation and/or particles convert the organic mantle of interstellar dust into amorphous hydrogenated carbon.

A set of data comparing UV photolysis and ion irradiation of ices showed that the yield of major products was similar for a simple ice containing $\mathrm{H}_{2} \mathrm{O}$ and $\mathrm{CO}_{2}$ (Gerakines et al 2000), showing that the ice chemistry seems to be quite similar whether induced by photons or charged particles. Indeed, HMT production has been reported when interstellar ice analogs containing $\mathrm{CH}_{3} \mathrm{OH}$ and $\mathrm{NH}_{3}$ are irradiated with protons (Cottin et al., 2001), the same as with photons. The main difference is that UV photons (typically at $122 \mathrm{~nm}$ ) only affect a few tenths of a micrometer in water-dominated ice, whereas protons can reach and alter the ice composition down to a few metres depth.

Chemical differentiation is more noticeable when molecules not dissociated by photons with a wavelength of $122 \mathrm{~nm}$ and above are involved in the chemical processes. This is the case for $\mathrm{CO}$ and $\mathrm{N}_{2}$. Different results are obtained if pure $\mathrm{CO}$ is photolyzed or proton irradiated (Gerakines and Moore 2001). Likewise $\mathrm{N}_{3}+$ is detected when ices containing $\mathrm{N}_{2}$ are proton irradiated, but not after photolysis (Hudson and Moore 2002). It seems that it is more the energy level than the way it is deposited (UV or charged particles) that matters for the chemistry.

We note that in these kinds of experiment, $\mathrm{C}_{3} \mathrm{O}_{2}$, which is sometimes evoked as an extended source of $\mathrm{CO}$, has been detected after irradiation of ices containing $\mathrm{CO}$ or $\mathrm{CO}_{2}$ (Brucato et al. 1997; Moore et al 1991). Kobayashi et al (1995) and Kasamatsu et al., (1997) were the first to report amino-acid production from irradiated ices. After an irradiation by $3-\mathrm{MeV}$ protons of mixtures containing water, ammonia and a carbon-containing molecule (carbon monoxide, methane or propane), they detected by HPLC several amino acids: glycine, and for the first time in a cometary simulation, alanine, aminobutiric acid and aminoisobutiric 
acid. These new detections were not made directly from the organic residue, but after an acid hydrolysis in water. Likewise for UV irradiation, only traces of glycine were found during the analysis of unhydrolyzed residues.

\subsubsection{Thermal Processing of Ices}

Polyoxymethylene and associated molecules and polymers have been detected when several mixtures containing formaldehyde, instead of being irradiated, were warmed slowly to room temperature (Schutte et al., 1993a, Schutte et al., 1993b).

There are many differences between the organics detected with or without UV processing of ices. Without irradiation, HMT is not detected, which is quite surprising as $\mathrm{H}_{2} \mathrm{CO}$ and $\mathrm{NH}_{3}$ readily react in the gaseous phase to form HMT (Bernstein et al., 1995; Walker 1964). Likewise, ketones, amides or esters, easily synthesized under irradiation, are quite rare in those thermal experiments. It seems that UV photons provide enough energy to surmount the energy barrier for formation of these molecules. Without UV, POM's production is favoured since it requires less energy.

\subsubsection{Relevance and Importance of Laboratory Simulations}

This section can not be concluded without discussing the relevance of experimental simulations. Of course, an irradiation of a few hours can not reproduce millions of years or more of slow evolution with complex heterogeneous chemistry in an interstellar environment that will never be completely reproduced in the laboratory. Nevertheless, in the 3.4- $\mu \mathrm{m}$ region, infrared spectra of methane and butane mixtures after a particle irradiation present a very good fit with the observations of dust particles in the diffuse interstellar medium (i.e. highly processed material), and even with spectra of residues from the Murchinson meteorite (Pendleton et al., 1994). The same results have been obtained with residues of $\mathrm{H}_{2} \mathrm{O}$ : $\mathrm{CO}: \mathrm{NH}_{3}: \mathrm{CH}_{4} / \mathrm{C}_{2} \mathrm{H}_{2} / \mathrm{CH}_{3} \mathrm{OH}$, which have been exposed to direct solar UV radiation on the EURECA space station (Greenberg and Li 1997, Greenberg et al., 1995). These are also highly processed materials. Thus Strazzulla's IPHAC appears to be similar to the refractory mantle of dust grains in the harsh conditions of the diffuse interstellar medium. The less-processed mantle formed in molecular clouds is almost certainly composed of the large range of molecules detected after experimental simulations, and the abundances of characteristic molecules like HMT or POM depends on the history of the grain and the relative contribution of the different energy sources: UV and proton irradiation lead to HMT, thermal processing to POM-like polymers. The importance of such simulations is underlined if one considers that data drawn from these simulations are necessary for the preparation of space missions to comets. A good illustration of this point is the selection and calibration of chromatographic columns for the COSAC experiment (ROSETTA mission - ESA) that requires an anticipation of the nature of the molecules to be searched for (see Sect. 8.6). 


\subsection{Origin and Evolution of Cometary Matter}

\subsubsection{Origin of Cometary Matter}

We have already seen (Sect. 8.1.3) how icy planetesimals populated the two present comet reservoirs, the Oort cloud and the Kuiper Belt, in the early times of the Solar System. We address now the problem of the formation of these icy planetesimals, and the processes determining the composition of cometary matter. As underlined by Yamamoto (1991) these two steps are distinct: the chemical composition is probably fixed to a large extent early in the accretion process, well before $\mathrm{km}$ sized bodies are built.

Figure 8.14 summarizes the present view of Solar System formation. All the matter originates in an interstellar cloud composed of dust and gas. Close to the protosun, towards the centre of the nebula, only rocky material would condense, while volatile species remained in the gas phase. Rocky planetesimals - bodies of intermediate size, ranging between $1 \mathrm{~m}$ and a few $\mathrm{km}$ - were formed there. These rocky planetesimals represent a crucial stage in the process of planet formation; they present a great similarity with present-day asteroids. On the contrary, towards the outermost parts of the nebula, where the temperature was lower, dust and ices formed the material of icy planetesimals, similar to present-day comets.

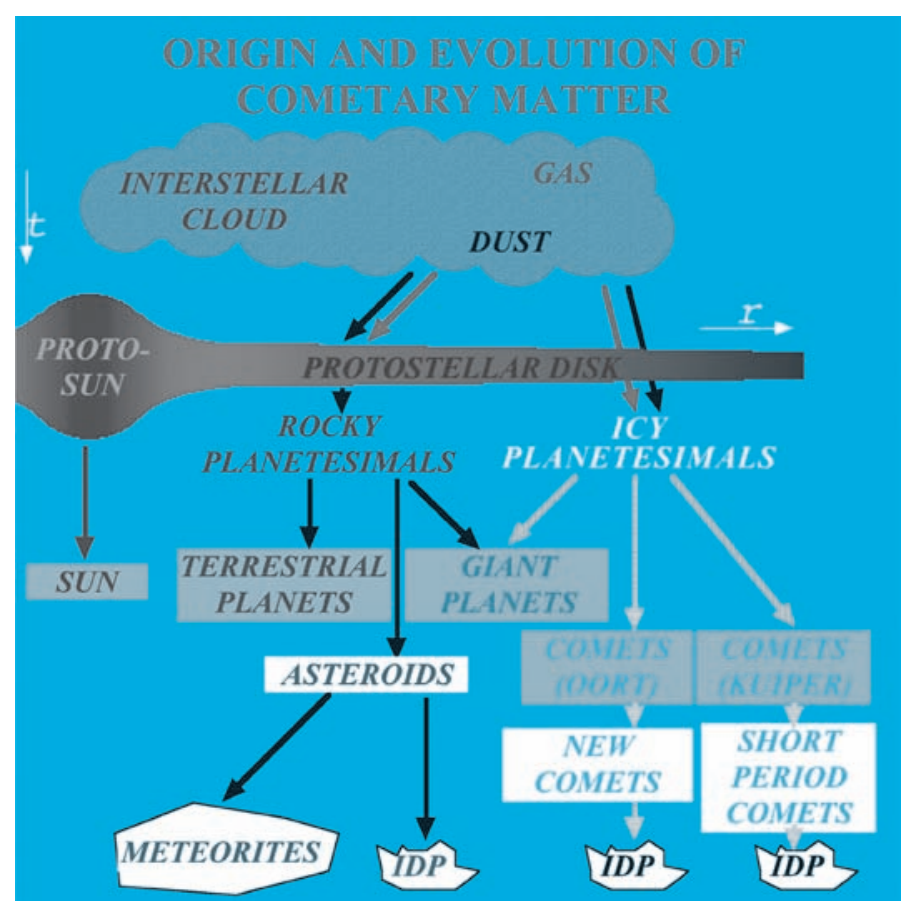

Fig. 8.14. Diagram showing the origin and the evolution of the cometary matter 
These rocky and icy planetesimals intervened in very different proportions in the formation of planets. Rocky planetesimals played the major role for the formation of the Earth and other telluric planets (Mercury, Venus, Mars), while it was mostly the icy planetesimals that took part in the formation of the cores of the giant planets Jupiter, Saturn, Uranus and Neptune. Today comets are thought to be a subset of these ancient icy planetesimals, which survived until now with no or very little evolution.

This global picture, displayed in Fig. 8.14, presents in fact several simplifications. For example, there is also a small number of short-period comets that come from the Oort cloud. Moreover, the separation between rocky and icy planetesimals is not that clear, because bodies of intermediate composition and properties have been found (e.g. Phaeton, Chiron). In addition bipolar flows (see Chap. 1, Part I by T. Montmerle), which are produced in the vicinity of the protosun, may reinject a certain quantity of matter from the protosolar nebula into the collapsing protostellar envelope, allowing material highly processed in the vicinity of the Sun to be incorporated into comets at great heliocentric distances. A similar effect is obtained in the model by Shu et al. (1996) where grains are ejected from the vicinity of the protosun towards the outer parts of the disk.

\subsubsection{Cometary Ices versus Interstellar Ices: the Facts}

In cold dense interstellar clouds, interstellar grains are coated with ice mantles whose composition can be observed indirectly in a few regions where they evaporate: hot cores surrounding a massive star in formation inside an interstellar cloud, such as occur, for example, in the heart of the Orion Nebula, or shocked regions along bipolar flows (e.g. Kurtz et al., 2000, Bachiller et al., 2001). In the first case, the ice mantles of the grains of the collapsing envelope are thermally evaporated close to the young massive star in formation. In the second case, it is the shock wave created by the bipolar flow propagating through the interstellar medium surrounding the young star that leads to the destruction of the ice mantles and releases the molecules to the gas phase. The good correlation of molecular abundances between cometary and interstellar ices (Table 8.4 and Fig. 8.15) is one of the arguments that leads us to conclude that the matter of comets is very close to interstellar matter, suggesting similar conditions of formation, and even possibly a direct link (comets being then a frozen piece of interstellar matter).

A more straightforward comparison should arise from direct measurements of interstellar ices. Unfortunately, these measurements can only be performed in front of strong infrared sources (e.g. Ehrenfreund and Charnley 2000 and references therein). Furthermore, identification of a precise species and quantitative determination of its abundance from its IR vibrational bands are more difficult than in the case of millimetre-wave rotation spectra of gas-phase species. Table 8.4 lists the abundances in two sets of young stellar objects (YSO), respectively leading to low-mass $\left(<2 M_{\text {Sun }}\right)$ and high-mass stars. The former case, 
Table 8.4. Cometary versus interstellar ices. Note their overall similarity. The agreement might be better in the case of ices surrounding low-mass protostars (a category to which the protosun belonged), but most of the present data are only upper limits, including $\mathrm{NH}_{3}$ and $\mathrm{CH}_{3} \mathrm{OH} . \mathrm{N}_{2}, \mathrm{O}_{2}, \mathrm{H}_{2}$ and rare gases are other important species whose ice abundances are not yet known. IS data are from Ehrenfreund and Charnley (2000); the range corresponds to 2 objects in each category (high-mass objects: W33A and N7538S; low-mass objects Elias16 and 29). Cometary data are from Table 8.1 (cometary volatiles); when no range is given, data refer to comet Hale-Bopp, except the $\mathrm{O}_{2}$ limit, which comes from comet Halley. X-CN is the sum of various CN species (mainly the $\mathrm{OCN}^{-}$ion in the case of IS ices)

\begin{tabular}{lccc}
\hline Species & $\begin{array}{c}\text { Interstellar ices } \\
\text { high-mass YSO }\end{array}$ & $\begin{array}{c}\text { Interstellar ices } \\
\text { low-mass YSO }\end{array}$ & $\begin{array}{c}\text { Cometary } \\
\text { volatiles }\end{array}$ \\
\hline $\mathrm{H}_{2} \mathrm{O}$ & 100 & 100 & 100 \\
$\mathrm{O}_{2}$ & $<7$ & - & $<0.5\left(^{*}\right)$ \\
$\mathrm{CO}$ & $9-16$ & $6-25$ & $1.7-23$ \\
$\mathrm{CO}_{2}$ & $14-20$ & $15-22$ & 6 \\
$\mathrm{CH}_{4}$ & 2 & $<1.6$ & 0.6 \\
$\mathrm{C}_{2} \mathrm{H}_{6}$ & $<0.4$ & - & 0.6 \\
$\mathrm{CH}_{3} \mathrm{OH}$ & $5-22$ & $<4$ & $0.9-6.2$ \\
$\mathrm{H}_{2} \mathrm{CO}$ & $1.7-7$ & - & $0.13-1.3$ \\
$\mathrm{HCOOH}$ & $0.4-3$ & - & 0.09 \\
$\mathrm{NH}$ & $13-15$ & $<9$ & 0.7 \\
$\mathrm{X}-\mathrm{CN}(*)$ & $1-3$ & $<0.4$ & $0.08-0.25$ \\
$\mathrm{OCS}, \mathrm{XCS}$ & $0.05-0.3$ & $<0.08$ & 0.4 \\
\hline
\end{tabular}

which corresponds to the Sun itself, is still very incomplete. $\mathrm{O}_{2}$ and $\mathrm{O}_{3}$, for which only upper limits have been measured, may contain a substantial fraction of atomic $\mathrm{O}$. The very volatile species $\mathrm{H}_{2}$ and $\mathrm{N}_{2}$, and rare gases like Ar, may be present to an unknown extent in IS ices as well as in cometary ices.

Table 8.5 gives the list of the 137 interstellar and circumstellar molecules (not including isotopic variants) detected to date (January 2004) in the gas phase. Among them are ethanol $\mathrm{C}_{2} \mathrm{H}_{5} \mathrm{OH}$, which has been long known, and glycolaldehyde $\mathrm{CH}_{2} \mathrm{OHCHO}$, whose detection has been recently claimed in the ISM (Hollis et al. 2000). Glycolaldehyde belongs to the family of molecules $\mathrm{C}_{n}\left(\mathrm{H}_{2} \mathrm{O}\right)_{n}$, which for $n>2$ are sugars or oses. In carbonaceous chondrites (the meteorites with composition closest to comets) Cooper et al. (2001) have detected several sugar molecules. If one also takes into account the presence of $\mathrm{H}_{2} \mathrm{CO}$, a sugar precursor at the per cent level in comets, that sugar molecules exist in comets is a likely hypothesis.

The heaviest known interstellar molecule to date is $\mathrm{HC}_{11} \mathrm{~N}$, which belongs to the family of cyanopolyynes (a succession of acetylene molecules linked one 


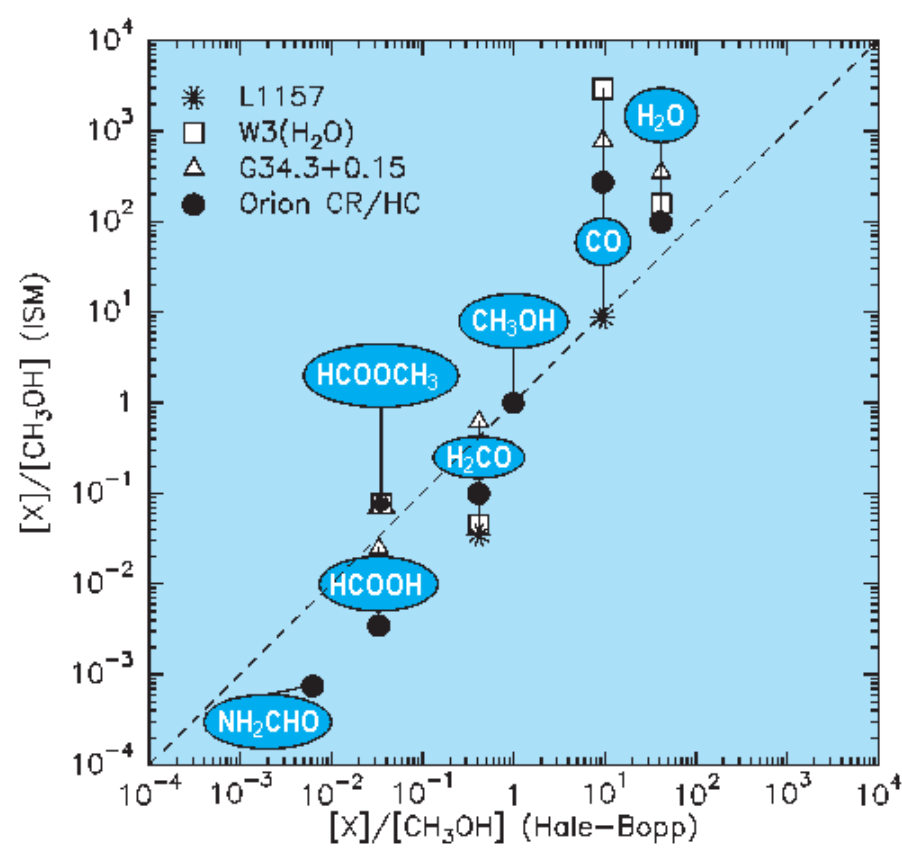

Fig. 8.15. Correlation of cometary (Hale-Bopp) and interstellar (ISM) gas-phase abundances (here for molecular species made of $\mathrm{C}$, $\mathrm{O}$, and $\mathrm{H}$ atoms; abundances are normalized to $\mathrm{CH}_{3} \mathrm{OH}$ ). The ISM regions selected for comparison are believed to release ice mantle molecules to the gas phase due to heat or shocks (Bockelée-Morvan et al., 2000)

to another to form a linear chain, and ending with a $\mathrm{CN}$ radical: $\left.\mathrm{H}(\mathrm{CC})_{n} \mathrm{CN}\right)$. The discovery of glycine $\mathrm{NH}_{2}-\mathrm{CH}_{2}-\mathrm{COOH}$ by Snyder et al., (e.g. 1997) in the ISM was not confirmed (Combes et al., 1996). Later searches by Ceccarelli et al., (2000) and Hollis et al., (2003) also led to upper limits. Recently, a detection has been published (Kuan et al., 2003) but it is also subject to debate. Due to the similarities between comets and the interstellar medium presented above, one can legitimately think that a number of these species exist also in comets; their detection is a challenge for new telescopes and space experiments (Sect. 8.6). In the interstellar medium grain surface chemistry also takes place. Charnley (1997) proposed a whole scheme of surface reactions to explain the formation of some complex compounds in the interstellar ices.

\subsubsection{Models of Cometary Matter and Comet Nucleus Formation}

According to some models, the rich IS chemistry may have been kept in comets, and thus be the ultimate source of some prebiotic compounds on Earth. This issue is especially important when discussing how common is prebiotic material 
Table 8.5. List of the 137 molecules detected in the interstellar or circumstellar medium (April 2003), after Wootten (2003) and PCMI website (Boulanger and Gérin). The reality of some detections (followed by "?") is still under discussion, e.g. glycine $\mathrm{NH}_{2} \mathrm{CH}_{2} \mathrm{COOH}$ (see text)

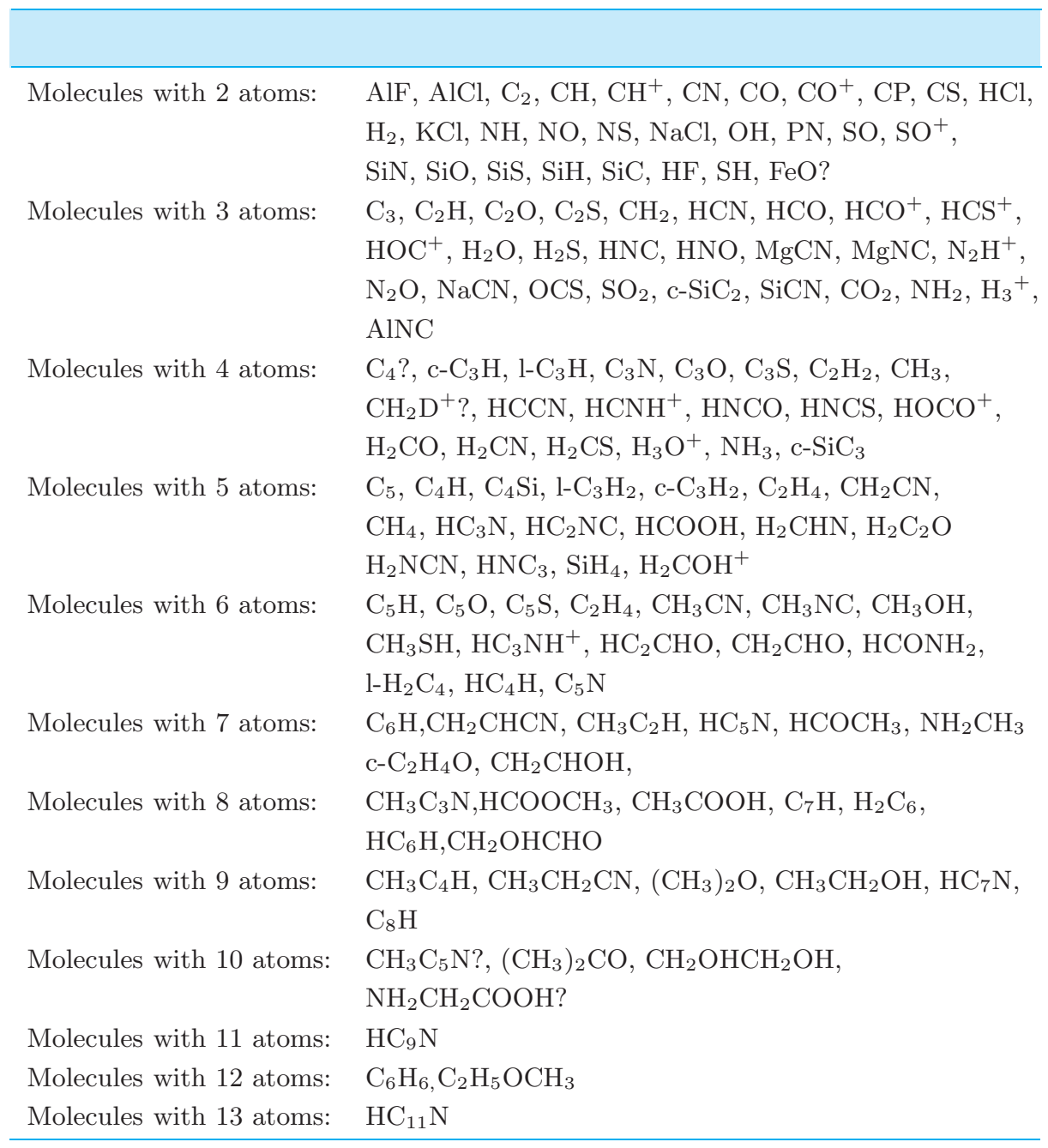

at the scale of our Galaxy, and the origin of chiral asymmetries (see Sect. 8.5.7 and the Chap. 3, Part II, by Cronin and Reisse). However, an important point is to realize that completely different and contradictory models are still proposed in this field, none of them being devoid of problems, although some are favoured by the present authors. A very detailed account of the pros and cons of each model can be found in Irvine et al., (2000a). 
Following Yamamoto (1991) we distinguish the formation of cometary matter from the formation of comet nuclei: to a large extent, the size increase of the icy bodies in the protosolar nebula is decoupled from their chemical evolution.

\subsubsection{Cometary Matter Formation}

The beginning of the accretion process is the most critical step in fixing the composition of cometary matter, as the small ( micrometre-sized) particles have the highest surface/volume ratio and maximize both surface reactions and gas-grain interactions. Furthermore, being small bodies $(\sim 1-10 \mathrm{~km})$, comets did not experience substantial heating (either internal, radioactive, or external, by collisions); these processes could, however, have affected the largest KBOs, a few $100 \mathrm{~km}$ in diameter (see Stern (2003) for a discussion of collisions in the Kuiper Belt, McKinnon 2002 and DeSanctis et al., (2001) for models of KBO thermal evolution). The three main categories of models for the first phase differ by the degree of reprocessing of the original material.

- In the interstellar model of comets, cometary matter is formed very early, with no or little further processing, by sticking together interstellar grains, and condensing to ice volatile molecules from the original interstellar gas phase to grain mantles. This is essentially the model proposed by Greenberg $(1982,1998)$ and retained by Irvine et al., (1980, 1996, 2000), Yamamoto (1983, 1985, 1991), Despois (1992), Bockelee-Morvan et al., (2000). In this model ice is mostly amorphous, except in the outer layer of the nucleus heated by the Sun when the comet approaches perihelion. The strong points in favour of this model are the coexistence of oxidized $\left(\mathrm{CO}_{2}, \mathrm{CO}\right.$, $\left.\mathrm{SO}_{2}\right)$ and reduced $\left(\mathrm{H}_{2} \mathrm{~S}, \mathrm{CH}_{4}, \mathrm{H}_{2} \mathrm{~S}\right)$ species, as observed in IS clouds, the rough overall abundances correlation (see previous section), including complex species like $\mathrm{HC}_{3} \mathrm{~N}, \mathrm{NH}_{2} \mathrm{CHO}, \mathrm{HCOOCH}_{3}$, and various indications of low-temperature processes (OPR, ortho:para ratios, in $\mathrm{H}_{2} \mathrm{O}$ and other species, deuterium enrichment, presence of very volatile species like $\mathrm{CO}$ and $\mathrm{CH}_{4}$ ). The syntheses occurred either in the interstellar cloud or during the protosolar phase, in the outer and cold part of the protosolar nebula, and bear testimony of ISM-like chemistry (low-temperature ion-molecule and surface reactions). Indeed the $\mathrm{DCN} / \mathrm{HCN}$ and $\mathrm{HDO} / \mathrm{H}_{2} \mathrm{O}$ ratios would mean in this hypothesis a formation temperature around 30-50 K (Meier et al., 1998), coherent with the OPR and $\mathrm{CO}$ volatility. Furthermore, this model is predictive to some extent, as the list of IS molecules proved to be a very good guide for the detection of new cometary species. The main problems are the presence in comets of crystalline silicates, not seen at present in the IS medium, and the deuteration level that, although high enough to imply that interstellar processes played a role, are much lower than the record values in hot cores.

- in the pure protosolar nebula model, or 'quenching' model (e.g. Prinn and Fegley 1989, Fegley 1993), the chemical memory has been fully or largely 
lost. This model is similar in many aspects to the successful models of the inner solar nebula (terrestrial planet zone). The comet-forming material is supposed to have experienced high temperatures in a hydrogen-rich nebula in thermochemical equilibrium (temperatures above $\sim 500 \mathrm{~K}$ ), which favours $\mathrm{CO}$ over $\mathrm{CH}_{4}$. The concept of "kinetic inhibition" of the $\mathrm{CO}$ to $\mathrm{CH}_{4}$ conversion (Lewis and Prinn 1980, Prinn and Fegley 1981) leads to quenched thermochemistry: the hot-gas composition remains in chemical equilibrium when the gas cools down, until a quenching temperature below which reactions become too slow and the composition remains unchanged. This allows CO to be the major C-bearing volatile, despite the higher thermodynamical stability of $\mathrm{CH}_{4}$ at low $T$. However, species predicted as abundant by the model are not observed $\left(\mathrm{NH}_{4} \mathrm{HCO}_{3}, \mathrm{NH}_{4} \mathrm{COONH} \mathrm{N}_{2}\right)$, the observed complex species like $\mathrm{HCOOCH}_{3}$ are not predicted, and explaining the cometary volatiles' chemical composition requires mixing contributions from IS gas, protosolar disk gas, and gas from the giant planet subnebulae in the right proportion (Prinn and Fegley 1989). Thus, an increasing complexity is required to explain even the gross features (e.g. oxidized/reduced species ratio) of the observed volatile composition.

- the partially reprocessed IS matter model is proposed by Lunine, Gautier and coworkers (e.g. Iro et al., 2003 and refs. therein). This aims at explaining several features of the Solar System besides cometary composition, such $\mathrm{D} / \mathrm{H}$ ratios and rare-gas abundances in the giant planets. The solar nebula is initially rather hot $(600 \mathrm{~K}$ at $1 \mathrm{AU})$ and cools down with the decrease of the disk accretion rate from $10^{-5}$ to $10^{-8} M_{\text {Sun }} / y r$. The nebula is supposed to be already isolated from the parent cloud (protostellar envelope). A key feature is that a significant fraction (depending on the heliocentric distance) of ice mantles of infalling interstellar grains vaporize through an accretion shock before reaching the nebula; the molecules then recondense as crystalline ice (as in the preceding pure protosolar nebula models), which incorporates minor species as gas clathrates or stoichiometric hydrates. The chemical composition of the gas is supposed to be unaltered, whereas the deuteration is diminished by interaction with the $\mathrm{H}_{2}$ of the nebula. Gas composition and clathrate/hydrate stoichiometry control the final composition of cometary ices (clathrates are a class of solids in which small gas molecules like $\mathrm{CH}_{4}, \mathrm{CO}_{2}$ or noble gases occupy "cages" made up of hydrogen-bonded water molecules; other species like $\mathrm{NH}_{3}$ form solid hydrates with well-defined composition $\left.\left(\mathrm{NH}_{3}\right)\left(\mathrm{H}_{2} \mathrm{O}\right)_{n}\right)$. Another essential feature is turbulence across the disk that can transport the silicate grains processed (crystallized) in the vicinity of the Sun to the cold regions where the ice forms.

\subsubsection{The Building of the Nuclei}

In the continuous accretion process leading from $0.1-\mu \mathrm{m}$ grains to km-sized bodies, there is a scale level, probably close to $1-10 \mathrm{~m}$, after which the chemical com- 
position of the matter is almost fixed (note, however, that very small molecules like CO could diffuse through the ice if warmed enough). At a larger size, most molecules are too deep inside to be affected by surface heating, energetic photons or particles (solar X or UV radiation, solar-wind particles and cosmic rays). Desorption processes and chemical reactions in the bulk of the ices, or on the probably large internal surface of this highly porous material, are limited, due to the very low temperature (a few tens of $\mathrm{K}$ to $150 \mathrm{~K}$ at most). Occasionally, collisions occurring during the accretion process may heat locally the cometesimals. The accretion of particles to form comets (or icy planetesimals) has been thoroughly studied by Weidenschilling (1997); his simulations show the formation of comet-size bodies from micrometre-size grains up to bodies $80 \mathrm{~km}$ in diameter in $2.5 \times 10^{5} \mathrm{yr}$.

\subsubsection{Are Today's Comets Like Comets in the Early Solar System?}

Were early comets similar to present ones? A given cometary nucleus is thought to have negligibly evolved since its formation, due to its low temperature. Exceptions may be a thin surface layer (due to particle and photon processing in the Oort cloud and the Kuiper Belt, and a few possible passages close to the Sun, see Mumma et al., 1993), and a slow volume loss of very volatile species (rare gases, CO). Occasional collisions may also have occurred in the Kuiper Belt and the Oort Cloud.

May chemistry inside the nucleus have altered the pristine composition of comets since their accretion? Navarro-Gonzalez et al., (1992) and NavarroGonzalez and Romero (1996) have studied theoretically the effect of ionizing radiation (cosmic rays and embedded radionuclides) on the cometary material and conclude that pristine organic compounds should not be altered except in the external layers of the comet. It is also predicted that an original enantiomeric excess of alanine would not be destroyed by radioracemisation during the decay of radionuclides. At the very most it should be attenuated, but comets could have contributed to the origin of chirality on Earth. The limitation of this work is that the kinetic data used in the calculations are for the liquid phase only, since no solid phase values are available; this may distort the result to some extent. The thermal racemization is a priori expected to be negligible at the comet low temperatures, but here also more theoretical and experimental work is required.

As stated before, the largest icy bodies $(>500 \mathrm{~km})$ in the Kuiper Belt might have for a limited time reached internally at some place conditions for melting to occur. This is, of course, a cause of chemical transformation.

If comets themselves are thought to have kept their original composition, their reservoirs have undergone at least a slow evolution in number and orbital parameters (e.g. Morbidelli et al., 2000). This affects directly the possibilities of delivery to the Earth. 


\subsection{Delivery to the Earth}

\subsubsection{Shooting Stars (Meteors)}

When a comet passes close to the Sun, the gases that are produced by the sublimation of the ice drag along the dust particles and release them into the interplanetary medium. Comets are thus one of the two principal sources of interplanetary dust (interplanetary dust particles, IDPs, also called "meteoroids"). The other source of IDPs is the collisions between rocky objects: asteroids or asteroid fragments. The largest cometary dust particles remain confined in the vicinity of the comet orbit and spread progressively along this orbit, forming a ring-like swarm of particles. In fact, planetary perturbations deform the orbits, which are no longer closed but transform into a tightly packed set of successive turns. Due to small differences in ejection conditions, individual particles follow different orbits, which, however, are close one to another and form a long ribbon through the Solar System. When the Earth crosses such a swarm, we experience a shower of shooting stars: during the entry into the Earth's atmosphere, particles larger than a size of about $1 \mathrm{~mm}$ produce, through heating, combustion and ionization of the air, a trail visible to the naked eye.

\subsubsection{Overall Picture of Matter Delivery to the Earth}

Several kinds of objects of the Solar System are likely to bring matter to the Earth: large-size bodies (asteroids or comets) responsible for more or less devastating impacts, small-size bodies (smaller than $10 \mathrm{~m}$ ) called meteoroids in general, and interplanetary dust for the smallest; if a fragment of these bodies reaches the ground, it is called a meteorite (or micrometeorite). This delivered material will affect the land, the oceans, the atmosphere and the terrestrial biosphere.

In Fig. 8.16 we present an overview of the different kinds of matter delivered to the Earth, displayed according to size. Around 0.1 micrometres are particles that are probably grains coming directly from the local interstellar medium surrounding the Sun. Above 10 micrometres the range of interplanetary particles starts. The smallest ones, around 10 micrometres, are decelerated and stopped in the stratosphere, where they can be collected by airplanes: these are the "stratospheric IDPs" previously mentioned. The slightly larger particles reach the ground and are thus called micrometeorites. If one considers the delivered mass (Fig. 8.17) and not the number of particles, the distribution presents a peak towards 100 micrometres. In the size range 10100 micrometres these particles produce in the atmosphere ion trails detectable by radar echoes; when the size exceeds about 100 micrometres (the value depending on the velocity of the particle) they appear in the visible as "shooting stars". With even larger particles, ranging from centimetre to decametre, one enters the field of the traditional meteorites. A few tens of metres is roughly 


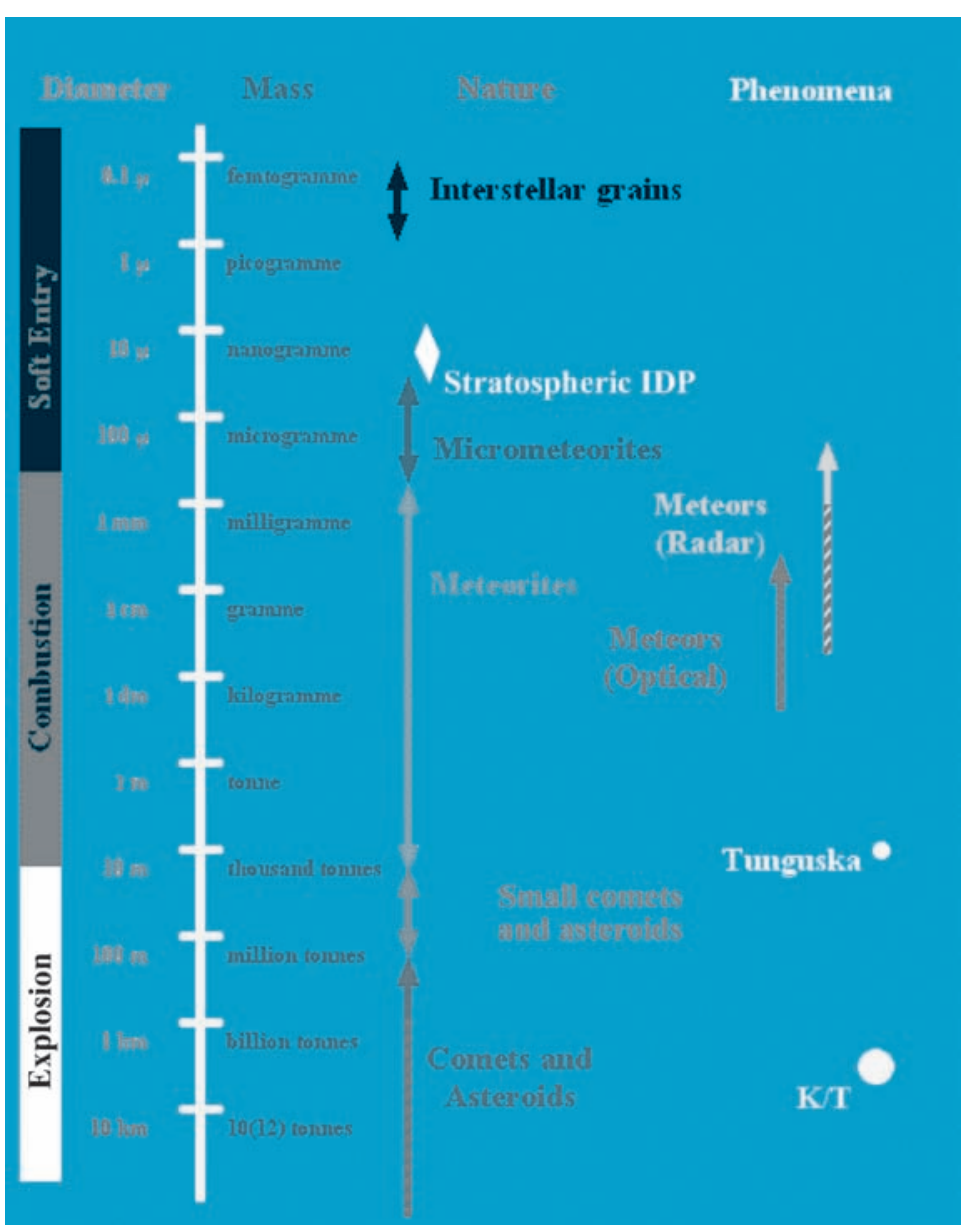

Fig. 8.16. Overview of particles delivered to Earth, displayed according to their size

the size of the bolide presumed to be responsible for the Tunguska explosion, which occurred in Siberia in June 1908, and blew down the forest over $2000 \mathrm{~km}^{2}$; the nature of the object is still debated, see Shuvalov and Artemieva 2002, Jopek et al., 2002 for recent discussions. At around ten kilometres diameter lies the object (comet or asteroid - e.g. Jeffers et al., 2001 - recent data favours the latter hypothesis, e.g. Kyte 1998) which would have been responsible, 65Myr ago, for producing the K-T sedimentary layer that marks in many places the boundary of the Cretaceous and the Tertiary geological periods, and of inducing massive species extinction, most notably that of dinosaurs (e.g. Frankel 1999; but the debate is still active, e.g. Stankel 2001, Facett et al., 2001, Le Loeuff and Laurent 2001). Such an impact occurs roughly every $100 \mathrm{Myr}$. 


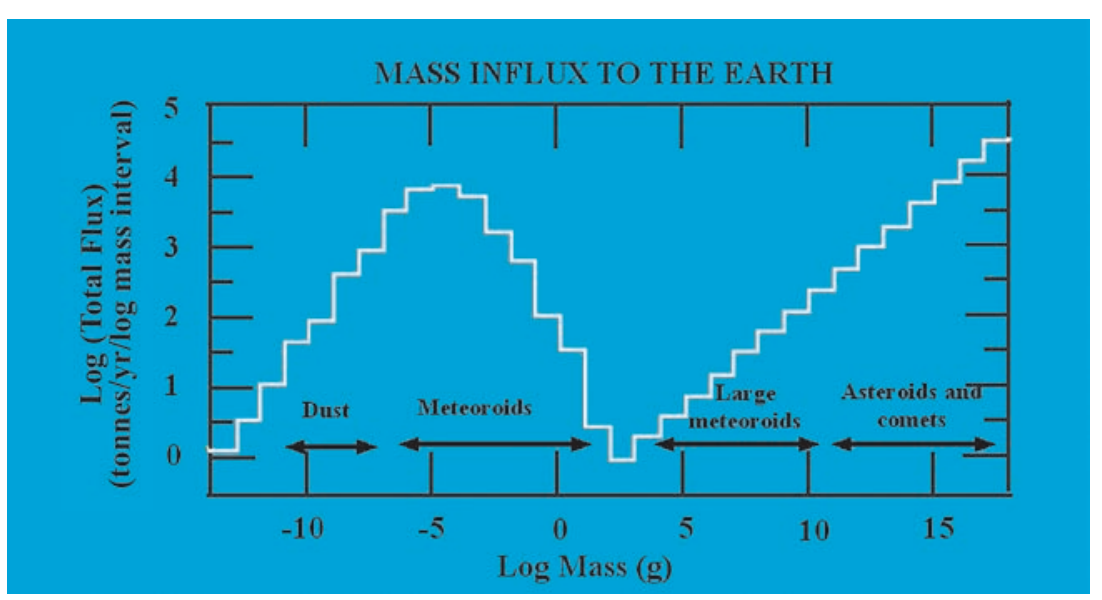

Fig. 8.17. Distribution in mass of the contributions to the Earth. The actual mean influx from the very largest objects is very uncertain. Rates are for present, and are believed to have been much higher in the first few 100 Myr of Earth's history (Steel 1996)

The micrometeorites represent a major contribution to the delivery of matter and especially of organic compounds and volatiles to the Earth; they may even be considered as a small prebiotic chemical reactor, as they contain many significant prebiotic compounds, including clays (catalyst), and insuring a natural confinement avoiding the dilution of the reactants (Maurette 1998, Maurette et al., 2000). The extrapolation of the micrometeoritic delivery in the past is highly uncertain, and ranges from conservative factors of 100 times the present rate to a high of $10^{6}$ times.

\subsubsection{Delivery of elements and Water}

According to different estimations, extraterrestrial delivery to Earth over 4.5 billion years ranges from $10^{21}$ to $7 \times 10^{23} \mathrm{~kg}$. The lower limit of this amount that can be attributed to comets has been estimated to be $10 \%$ by Chyba et al., (1990).

\section{- $\mathrm{H}_{2} \mathrm{O}$}

It has been suggested that most of the water of the Earth oceans $\left(1.4 \times 10^{9} \mathrm{~km}^{3}\right.$, roughly $10^{6}$ times comet Halley mass) could have been imported by comet impacts during a period of heavy bombardment (e.g. Delsemme 2000). Recent dynamical models of the early Solar System (Morbidelli et al., 2000), and recent measurements of the $\mathrm{D} / \mathrm{H}$ in cometary water (to be compared with $\mathrm{D} / \mathrm{H}$ in the oceans $($ SMOW $)$ ) (Dauphas et al., 2000) both lead to a maximum of $\sim 10 \%$ of 
water of cometary origin; the latter argument assumes that the $\mathrm{D} / \mathrm{H}$ ratio measured in 3 comets is typical of all comets. Petit and Morbidelli discuss further the delivery of water in their chapter. An even lower limit (1\%) on cometary $\mathrm{H}_{2} \mathrm{O}$ in ocean water has been recently presented, from indirect arguments on rare gases and metals (Dauphas and Marty 2002).

- Carbon

Carbon in comets is roughly $18 \%$ by mass. Chyba et al., (1990) estimated that the contribution of comets to the terrestrial carbon inventory ranges from $2 \times 10^{19}$ to $10^{22} \mathrm{~kg}$; while the total budget of carbon on Earth is about $9 \times 10^{19} \mathrm{~kg}$. Even if uncertainties linked to the total amount of comets impacting the Earth since its formation are not lifted, those estimations show that they brought a significant amount of carbonaceous material. The extent to which organic molecules can survive cometary impact is discussed in Sect. 8.5.5.

\section{- Other Elements}

The other elements required for a protocell formation are also present on comets. Phosphorus, which is involved in the synthesis of sugars, has been detected by mass spectroscopy $(m / e=31)$ in grains of comet Halley by the PUMA mass spectrometer on board Vega 1. But its abundance is very low and detection may have been affected by interference from ions such $\mathrm{CH}_{2} \mathrm{OH}^{+}$(Kissel and Krueger 1987). However, analysis by laser probe mass spectroscopy of interplanetary dust particles (IDPs), whose cometary origin is probable, has led to the detection of $\mathrm{PO}_{2}$ and $\mathrm{PO}_{3}$ anions (Radicati-Di-Brozolo et al 1986). Ni and Fe have been detected in comet Ikeya-Seki and Halley.

\subsubsection{Prebiotic Molecules from Comets?}

Five families of compounds are considered to be the key prebiotic monomers required before starting a chemical evolution from which life would arise. These are amino acids (whose combinations lead to proteins), purine bases (adenine and guanine), pyrimidines bases (cytosine, uracil and thymine), sugars (whose association lead to RNA and DNA molecules), and fatty acids (cell walls). It is very interesting to note that, even if these compounds have not been detected in comets (maybe because of limitations in the sensitivity of telescopes, but also essentially because the synthesis of most of them requires liquid water), all but thymine can be easily produced in an early Earth environment from cometary precursors such as $\mathrm{HCN}, \mathrm{HC}_{3} \mathrm{~N}, \mathrm{H}_{2} \mathrm{CO}$ and $\mathrm{CO}$ that have been firmly detected in cometary comae (Table 8.6).

Nevertheless, complex compounds of exobiological interest may also be present in comets. The only detection of adenine is a very tentative interpretation of PUMA's mass spectra of comet Halley's dust (Kissel and Krueger 1987). Such a molecule could be synthesized by HCN condensation without any 
Table 8.6. Prebiotic syntheses from cometary molecules, from (Oró and Cosmovici 1997)

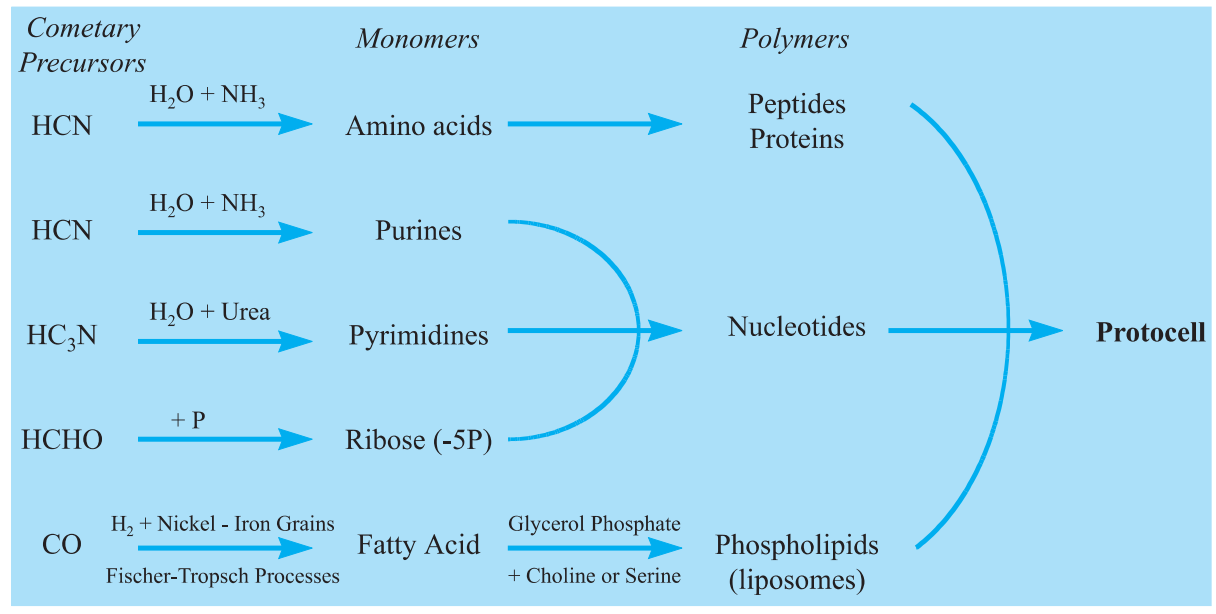

liquid water (Oro and Cosmovici 1997, Wakamutsu et al., 1966). Moreover, as already seen in this chapter, when ices relevant to the composition of interplanetary dust particles or comets are irradiated, complex molecules are formed, and among them, the simplest amino acid, glycine (Briggs et al., 1992). After an acid hydrolysis in water other amino acids, such as alanine, valine, proline and others, have also been identified (Bernstein et al., 2002; Kobayashi et al., 1995; Muñoz Caro et al., 2002).

\subsubsection{Do Molecules Survive From Comets to the Earth?}

Thus, comets may have imported prebiotic elements to early Earth, which, when mixed with liquid water, may have allowed the synthesis of all molecules thought to be necessary for the origin of life. To date, it is not clear as to whether the importation of such key compounds occurs at the simple stage of HCN, $\mathrm{HC}_{3} \mathrm{~N}, \mathrm{HCHO} \ldots$, or more elaborate species such as amino acids, puric and pyrimidic acids and others. But before seeding the oceans, those compounds have to survive:

- if brought by small dust particles, they are subject in the interplanetary medium to the action of solar UV radiation, solar-wind particles and galactic cosmic rays; while the comet particle is slowed down and warmed up by the Earth's atmosphere, they must resist pyrolysis (destruction at very high temperature), and chemical reactions in the atmosphere. If they reach the ground, they should also survive the ensuing impact.

- alternatively, if the nucleus impacts directly the Earth, can the molecule survive the dramatic energy release? 
Experiments conducted in space have shown that amino acids are quite unstable to solar UVs, but also that when somehow shielded in minerals, such as meteoritic powder, they can survive in space, and undergo no racemization (Barbier et al., 2002; Boillot et al., 2002).

The case of impacts of large bodies has been theoretically treated by Chyba et al (1990). It appears that organic compounds (even amino acids, if present) contained in cometary impactors of 100 to $200 \mathrm{~m}$ in size could survive a collision with Earth in a 10-bar $\mathrm{CO}_{2}$ atmosphere thanks to an efficient aerobreaking. Comparisons with Venus and Mars lead us to think that it is the most probable composition of the primitive atmosphere. The authors note in their conclusion that "It is intriguing that it is exactly these dense $\mathrm{CO}_{2}$ atmospheres, where photochemical production of organic molecules should be the most difficult, in which intact cometary organics would be delivered in large amounts". Indeed, organic syntheses are efficient in reduced atmospheres, and even in weakly reduced atmospheres such as that of Titan $\left(\mathrm{N}_{2}, \mathrm{CH}_{4}\right)$, whereas they are very difficult in the oxidized environment that must have been the early Earth's atmosphere.

On the experimental scene, Blank et al (2001) conducted a series of shock experiments to assess the feasibility of the delivery of amino acids to the Earth via cometary impacts. It appears that a large fraction of the amino acids do survive impacts. It has also been shown that some chemistry occurs, which leads to the formation of peptide bonds and new compounds including amino-acid dimers.

\subsubsection{Comparison of Comets with Other Likely Sources of Prebiotic Molecules}

Figure 8.17 displays the total mass delivered to the Earth for each mass interval (note that these intervals are logarithmic). Two main contributions show up, which are very different in nature: while the small particles could more or less softly deliver molecules to the Earth atmosphere and surface, large-size impactors bring also large quantities of matter but of course in a very different - discontinuous and catastrophic - mode. The very high temperatures reached by gases allow the synthesis of some compounds, as well as leading to their destruction as discussed in the previous section.

In the so-called intermediate atmospheres (in which the main gases are e.g. $\mathrm{CO}_{2}, \mathrm{H}_{2} \mathrm{O}, \mathrm{N}_{2}$ instead of $\mathrm{NH}_{3}$ and $\mathrm{CH}_{4}$ ) extraterrestrial delivery plays a major role in the total budget of prebiotic molecules (Chyba and Sagan 1996, Jenniskens et al., 2000). Table 8.7 gives in $\mathrm{kg} / \mathrm{yr}$ the quantity of these molecules brought by various processes in the case of reducing and neutral (or moderately oxidizing) atmospheres. The large difference between these two types of atmosphere is noticeable, in particular for the synthesis of molecules by the shock waves induced by impacts. It should be noted that if many experiments have been undertaken in the laboratory on the chemical syntheses induced by electric discharges or energetic photons, the experimental studies relevant to the entry of particles in the atmosphere are very difficult and are still in a much less elaborate stage. The direct observation of molecules deposited by the meteors in the 
Table 8.7. Major sources of prebiotic organics for two candidate early Earth atmospheres. The intermediate atmosphere corresponds here to a ratio $\mathrm{H}_{2} / \mathrm{CO}_{2}=0.1$. Note the considerably larger synthesis efficiency in the case of reducing atmospheres, which are those used by Urey and Miller in their experiments. In the more probable case of an intermediate atmosphere, external delivery is a major source of organics, as $30 \%$ come from IDPs. After Chyba and Sagan (1996) for the atmospheric processes, and Ehrenfreund et al., (2002) for the hydrothermal vents and comet impact delivery estimations

\begin{tabular}{lll}
\hline Source & $\begin{array}{l}\text { Reducing atmosphere } \\
(\mathrm{kg} / \mathrm{yr})\end{array}$ & $\begin{array}{l}\text { Intermediate atmosphere } \\
(\mathrm{kg} / \mathrm{yr})\end{array}$ \\
\hline UV photolysis & $1 \times 10^{12}$ & $3 \times 10^{8}$ \\
Electric discharge & $3 \times 10^{9}$ & $3 \times 10^{7}$ \\
IDPs & $2 \times 10^{8}$ & $2 \times 10^{8}$ \\
Shocks from impacts & $2 \times 10^{10}$ & $4 \times 10^{2}$ \\
Shocks from meteors & $4 \times 10^{9}$ & $8 \times 10^{1}$ \\
K/T extrapolation & $2 \times 10^{8}$ & $2 \times 10^{8}$ \\
Total (atmosphere) & $1 \times 10^{12}$ & $7 \times 10^{8}$ \\
Hydrothermal vents & $1 \times 10^{8}$ & $1 \times 10^{8}$ \\
Comet impact delivery & $1 \times 10^{11}$ & $1 \times 10^{11}$ \\
\hline
\end{tabular}

atmosphere has been attempted during the recent Leonid shower (CN: Rairden et al., 2000, HCN: Despois et al., 2000).

\subsubsection{Chiral Molecules: from the Interstellar Medium to the Early Earth?}

Among the most intriguing questions related to the origin of life on Earth is the origin of the chirality for some key molecules: amino acids (configuration L) and sugars (configuration D).

Even if the cometary contribution were modest in mass, it might still have played a remarkable role, allowing via cometary dust particles or impact to directly forward to the early Earth some interstellar molecules, which could have played an important part in the apparition of homochirality (e.g. Bonner et al., 1999 and references therein). Indeed, an enantiomeric excess has been measured for some nonbiological amino acids in meteorites (see Cronin and Reisse Chapt. 3, Part II). Therefore, it is conceivable that an excess was also present for the biological amino acids (for which analysis is much more difficult due to the risk of contamination by terrestrial biomolecules). Such an excess could have been transmitted to the early Earth, and constitute an initial asymmetry later amplified by prebiotic chemistry (see Commeyras et al., 2004). The possible importance of this scenario is the fact that abiotic mechanisms able to produce nonracemic mixtures are very rare. 
Which physical or chemical mechanism could then have led to a nonracemic mixture in the meteorites? Bailey and collaborators highlighted conditions favourable for the buildup of chiral asymmetry in the interstellar medium, and more especially in regions of star formation (Bailey et al., 1998, Bailey 2001). They detected the presence of circularly polarized infrared radiation in vast regions, which could easily include a whole Solar System in formation. This suggests the possible existence in these same regions of circularly polarized ultraviolet radiation, which still has to be detected. This circularly polarized ultraviolet radiation could lead to a differential synthesis or destruction of one enantiomer with respect to the other. If conditions similar to those in Orion prevailed at the time of formation of our Solar System, and if these interstellar molecules could actually be preserved till their integration into the matter of the protosolar nebula, this could be a plausible and relatively natural scenario for L/D symmetry breaking between enantiomers. This scenario seems at least as probable as the assumption of a fortuitous encounter of the protosolar nebula with a neutron star (a pulsar), also suggested as a likely source of polarized light. In comets, the search for nonracemic mixtures will require in situ chemical analysis or laboratory analysis of returned samples.

\subsection{Ground-based and Space Exploration of Comets: New Developments}

In the Solar System, several major space missions relate to comets. ESA is preparing the ROSETTA probe that will reach comet $69 \mathrm{P} /$ ChuryumovGerasimenko (the previous target, $\mathrm{P} /$ Wirtanen, had to be abandoned as the launch was delayed); after a ten-year cruise, it will meet the comet and follow it for almost two years, during which a lander will be deposited on the surface. On the lander, the COSAC experiment (e.g. Rosenbauer et al., 1999) will perform a chemical analysis of cometary volatiles by gas chromatography of samples taken after drilling down to $20 \mathrm{~cm}$ below the surface. With a detection limit of $10^{-5}-10^{-6}$ with respect to water, more than 100 cometary species might be detected (Szopa et al., (2003) and Crovisier (2004)). The use of chiral columns will allow the measurement of enantiomeric excess.

A US mission, STARDUST, aims to collect thousand of cometary grains and bring them back to Earth. Another probe, CONTOUR, intended to tour 3 cometary nuclei, seems unfortunately to have been definitively lost. Table 8.8 summarizes all the space efforts directed towards comets in the next few years. As for ground-based observers, they have to wait for a new bright comet, like the recent comets Hyakutake and Hale-Bopp. In the near future, large ground-based telescopes, like the Atacama Large Millimeter Array (64 antennas, $12 \mathrm{~m}$ in diameter, operating as an interferometer in a very good high altitude astronomical site in Chile), the Large Millimeter Telescope (50-m antenna), the Green Bank 


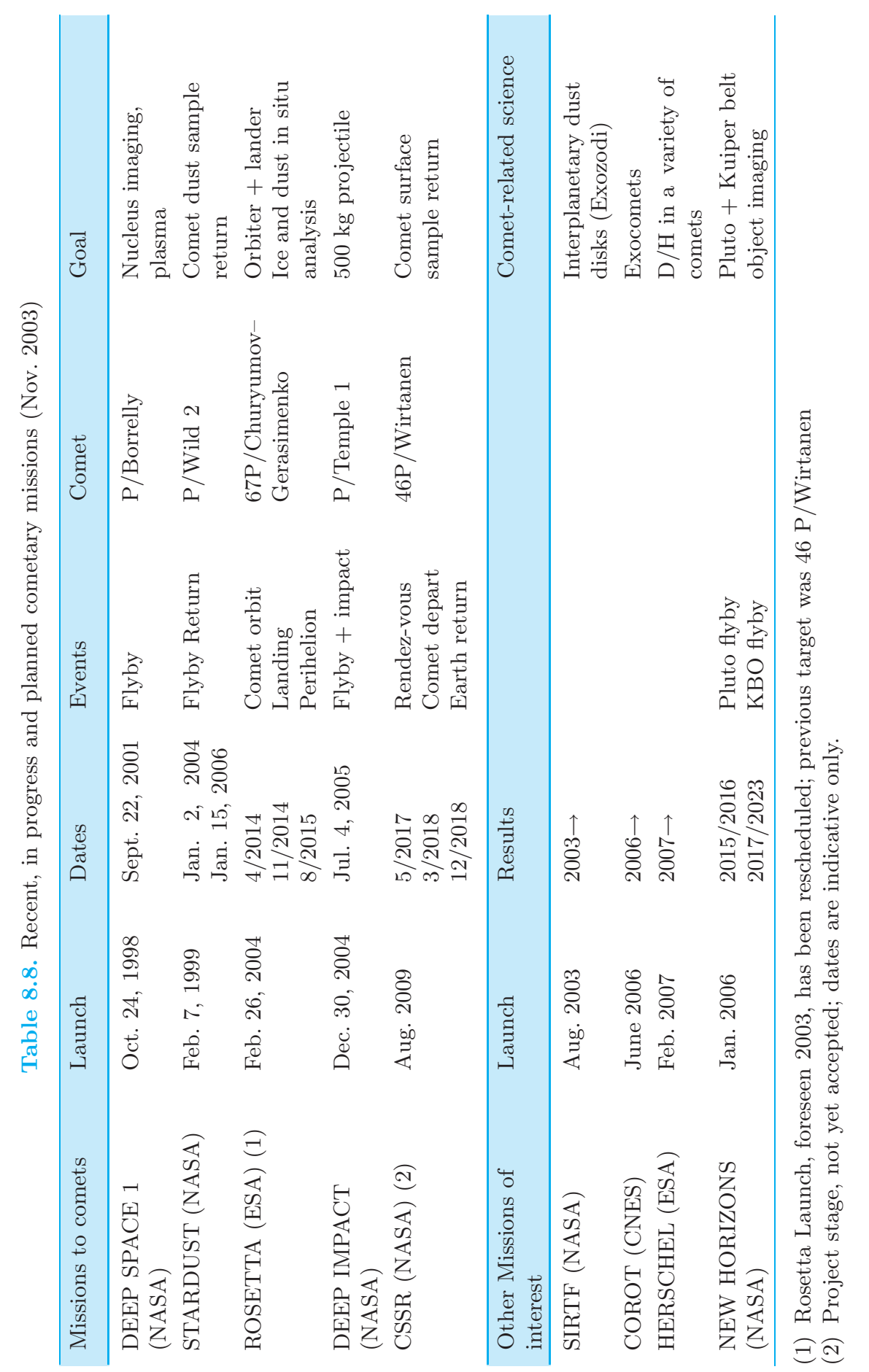


Telescope (100m antenna) will bring higher sensitivity and space resolution. Out of the Solar System, the Corot mission should make it possible to detect comets around other stars (Lecavelier des Etangs et al. 1999).

\subsection{Conclusion}

Comets can bring down to the Earth elements like carbon, as well as water (in an amount that remains to be determined). Moreover, they contain simple organic molecules, and it is probable that they contain more complex ones. Thus comets are likely candidates for early Earth enrichment in prebiotic molecules, either through the dust particles they release - some of which end up encountering the Earth atmosphere - or through the atmospheric chemical syntheses - and possibly also through direct delivery - taking place when cometary nuclei impact the Earth. The same impacts may also strongly affect the later development of life, either locally, or on the scale of the planet.

Many questions are central to the current debates, which we hope will find a response in coming years, and for which space missions can bring important results:

- Where and when were formed the cometary matter, and then the comets?

- How close is cometary matter to interstellar matter?

- Do comets contain amino acids and other bricks of life?

- Do these molecules present an enantiomeric excess, or are they in racemic mixture?

- What is the precise budget of the cometary contributions to the Earth (fraction of the water of the oceans; fraction of carbon; fraction of prebiotic molecules)?

- What is the global effect of comets on life, its appearance and its development?

Out of the Solar System, is the presence of an Oort cloud or a Kuiper Belt an exceptional characteristic of our Solar System or a banal fact among main sequence stars? Given the crucial part potentially played by comets in the origin and the evolution of life on Earth, it appears fundamental to know the probability for a star with habitable planets to be also surrounded by comets. New research topics thus develop on these exocomets (comets orbiting around other stars). The properties of the spectra of a close star, $\beta$ Pictoris - one of the rare main sequence stars around which a disc has been detected - are currently convincingly interpreted as due to a constant infall of "evaporating bodies" on this star (Lagrange et al., 2000, Karmann et al., 2001, 2003). Much is then to be expected from ambitious space experiments under development, like the Corot mission (Baglin 2003), which should make it possible to detect directly comets, as well as Earth-size planets around a wide range of other stars (Lecavelier des Etangs et al., 1999, Bordé et al., 2003). 


\section{Acknowledgement}

We are very grateful for detailed reading, precious comments and many corrections on this text by W. Irvine, J. Crovisier, M. Gargaud, B. Barbier and J. Reisse.

\section{References}

A'Hearn, M. F., Millis, R. L., Schleicher, D. G., Osip, D. J., Birch, P. V. (1995). The ensemble properties of comets: Results from narrowband pghotometry of 85 comets, 1976-1992. Icarus, 118, 223-270.

A'Hearn, M.F., Boehnhardt, H., Kidger, M., West, R.M. eds (1999). Proceedings of the first international conference on Comet Hale-Bopp. Earth, Moon and Planets, $\mathbf{7 7}, 78 \& 79$.

Allamandola, L. J., Sandford, S. A., Valero,G. J. (1988). Photochemical and thermal evolution of interstellar/precometary ice analogs. Icarus, 76, 225-252.

Altwegg, K., Balsiger, H., Geiss, J. (1999). Composition of the Volatile Material in Halley's Coma from In Situ Measurements. Space Science Reviews, 90, 3-18.

Altwegg, K., Bockelée-Morvan, D. (2003). Isotopic Abundances in Comets. Space Science Reviews, 106, 139.

Arpigny, C., Jehin, E., Manfroid, J., Hutsemékers, D., Schulz, R., Stüwe, J. A., Zucconi, J., Ilyin, I. (2003). Anomalous Nitrogen Isotope Ratio in Comets, Science, 301, 1522.

Bachiller, R., Pérez Gutiérrez, M., Kumar, M. S. N., Tafalla, M. (2001). Chemically active outflow L 1157, Astronomy and Astrophysics, 372, 899.

Baglin, A. (2003). COROT: A minisat for pionnier science, asteroseismology and planets finding. Advances in Space Research, 31, 345.

Bailey, J. (2001). Astronomical Sources of Circularly Polarized Light and the Origin of Homochirality. Origins of Life and Evolution of the Biosphere, 31, 167.

Bailey, J., Chrysostomou, A., Hough, J. H., Gledhill, T. M., McCall, A., Clark, S., Menard, F., Tamura, M. (1998). Circular Polarization in Star-Formation Regions: Implications for Biomolecular Homochirality, Science, 281, 672.

Ball, A. J., Gadomski, S., Banaszkiewicz, M., Spohn, T., Ahrens, T. J., Whyndham, M., Zarnecki, J. C. (2001) An instrument for in situ comet nucleus surface density profile measurement by gamma ray attenuation, Planetary and Space Science, 49, 961.

Barbier, B., Henin, O., Boillot, F., Chabin, A., Chaput, D., Brack, A. (2002). Exposure of amino acids and derivatives in the Earth orbit. Planet. Space Sci., 50, 353-359.

Benkhoff, J. (1999). On the Flux of Water and Minor Volatiles from the Surface of Comet Nuclei, Space Science Reviews, 90, 141.

Bernstein, M. P., Dworkin, J. P., Sandford, S. A., Cooper, G. W.,Allamandola, L. J. (2002). Racemic amino acids from the ultraviolet photolysis of interstellar ice analogues. Nature, 416, 401-403.

Bernstein, M. P., Allamandola, L. J., Sandford, S. A. (1997). Complex organics in laboratory simulations of interstellar/cometary ices. Adv. Space Res., 19, 991-998.

Bernstein, M. P., Sandford, S. A., Allamandola, L. J., Chang, S. (1994). Infrared spectrum of matrix isolated Hexamethylenetetramine in $\mathrm{Ar}$ and $\mathrm{H}_{2} \mathrm{O}$ at cryogenic temperatures. J. Phys. Chem., 98, 12 206-12 210. 
Bernstein, M. P., Sandford, S. A., Allamandola, L. J., Chang, S., Scharberg, M. A. (1995). Organic Compounds Produced By Photolysis of Realistic Interstellar and Cometary Ice Analogs Containing Methanol. Astrophys. J., 454, 327-344.

Biver et al., (1999) Long-Term Evolution of the Outgassing of Comet Hale- Bopp from Radio Observations. Earth, Moon and Planets, 78, 5-11.

Biver, N., Bockelée-Morvan, D., Crovisier, J., Colom, P., Henry, F., Moreno, R., Paubert, G., Despois, D., Lis, D. C. (2002a) Chemical Composition Diversity Among 24 Comets Observed At Radio Wavelengths. Earth Moon and Planets, 90(1), 323-333.

Biver, N., Bockelée-Morvan, D., Colom, P., Crovisier, J., Henry, F., Lellouch, E., Winnberg, A., Johansson, L. E. B., Gunnarsson, M., Rickman, H., Rantakyrö, F., Davies, J. K., Dent, W. R. F., Paubert, G., Moreno, R., Wink, J., Despois, D., Benford, D. J., Gardner, M., Lis, D. C., Mehringer, D., Phillips, T. G., Rauer, H. (2002b). The 1995-2002 Long-Term Monitoring of Comet C/1995 O1 (HALE-BOPP) at Radio Wavelength, Earth Moon and Planets, 90, 5.

Blank, J. G., Miller, G. H., Ahrens, M. J., Winans, R. E. (2001). Experimental Shock Chemistry of Aqueous Amino Acid Solutions and the Cometary Delivery of Prebiotic Compounds. Orig. Life, 31, 15-51.

Bockelée-Morvan, D. (1997). Cometary Volatiles: the status after comet C/1996 B2 Hyakutake. In Molecules in Astrophysics: Probes and Processes, IAU symposium 178, E.F. van Dishoeck ed., Kluwer, p. 219.

Bockelee-Morvan, D., Brooke, T.Y., Crovisier, J. (1995) On the origin of the 3.2 to 3.6-micrometre emission features in comets, Icarus, 116, 18.

Bockelée-Morvan, D. Crovisier, J. (2002). Lessons of Comet Hale-Bopp for Coma Chemistry: Observations and Theory. Earth Moon and Planets, 89, 53-71.

Bockelée-Morvan, D., Rickman, H. (1999). C/1995 O1 (Hale-Bopp): Gas Production Curves and Their Interpretation. Earth Moon Plan., 79, 55-77.

Bockelée-Morvan, D., Biver, N., Moreno, R., Colom, P., Crovisier, J., Gérard, E., Henry, F., Lism, D. C., Matthews, H., Weaver, H. A., Womack, M., Festou, M. C. (2001). Outgassing Behaviour and Composition of Comet C/1999 S4 (LINEAR) During Its Disruption. Science, 292, 1339-1343.

Bockelée-Morvan, D., Gautier, D., Lis, D. C., Young, K., Keene, J., Phillips, T. G., Owen, T., Crovisier, J., Goldsmith, P. F., Bergin, E.A., Despois, D., Wootten, A. (1998). Deuterated Water in Comet C/1996 B2 (Hyakutake) and its Implications for the Origin of Comets. Icarus, 133, 147-162.

Bockelée-Morvan D., Lis D. C., Wink J. E., Despois D., Crovisier J., Bachiller R., Benford D. J., Biver N., Colom P., Davies J. K., Gérard E., Germain B., Houde M., Mehringer D., Moreno R., Paubert G., Phillips T. G., Rauer H. (2000). New molecules found in comet C/1995 O1 (Hale-Bopp). Investigating the link between cometary and interstellar material., Astronomy and Astrophysics, 353, 1101-1114.

Bockelée-Morvan, D., Crovisier, J., Mumma, M., Weaver, H. (2004, in press), The Composition of Cometary Volatiles, in Comets II, eds. M. Festou, U. Keller, H. Weaver, Univ. of Arizona Press.

Boehnhardt, H. (2002), Comet Splitting - Observations and Model Scenarios, Earth Moon and Planets, 89, 91.

Boillot, F., Chabin, A., Buré, C., Venet, M., Belsky, A., Bertrand-Urbaniak, M., Delmas, A., Brack, A., Barbier, B. (2002). The Perseus Exobiology Mission on MIR: Behaviour of Amino Acids and Peptides in Earth Orbit. Orig. Life, 32, 359-385. 
Bonner, W. A., Mayo Greenberg, J., Rubenstein, E. (1999). The Extraterrestrial Origin of the Homochirality of Biomolecules - Rebuttal to a Critique, Origins of Life and Evolution of the Biosphere, 29, 215.

Bordé, P., Rouan, D., Léger, A. (2003). Exoplanet detection capability of the COROT space mission, Astronomy and Astrophysics, 405, 1137.

Boulanger, F., Gerin, M. Interstellar and Circumstellar Molecules, http://www.lra.ens.fr/ pcmi/table-mol-an.html.

Bouwman, J., de Koter, A., Dominik, C., Waters, L.B.F.sM. (2003). The origin of crystalline silicates in the Herbig Be star HD 100546 and in comet Hale-Bopp. Astronomy and Astrophysics, 401, 577.

Briggs, R., G. Ertem, J. P. Ferris, J. M. Greenberg, P. J. McCain, C. X. MendozaGomez, W. Schutte (1992). Comet Halley as an aggregate of interstellar dust and further evidence for the photochemical formation of organics in the interstellar medium. Orig. Life, 22, 287-307.

Brucato, J.R., Colangeli, L., Mennella, V., Palumbo, P., Bussoletti, E. (1999). Silicates in Hale-Bopp: hints from laboratory studies. Planetary and Space Science, 47, $773-779$

Brucato, J.R., Castorina, A.C., Palumbo, M.E., Satorre, M.A., Strazzulla, G. (1997). Ion Irradiation and Extended CO Emission in Cometary Comae. Planetary and Space Science, 45, 835-840.

Capria, M. T. (2002). Sublimation Mechanisms of Comet Nuclei, Earth Moon and Planets. 89, 161 .

CBAT. Cometary Designation System. http://cfa-www.harvard.edu/iau/lists/CometResolution.html.

Ceccarelli, C., Loinard, L., Castets, A., Faure, A., Lefloch, B. (2000). Search for glycine in the solar type protostar IRAS 16293-2422. Astronomy and Astrophysics, 362, $1122-1126$.

Chamberlin, T. C., Chamberlin, R. T. (1908). Science, 28, 897.

Charnley, S.B. (1997). On the nature of interstellar organic chemistry. In Astronomical and Biochemical Origins and the Search for Life in the Universe, 5th Int. Conf. On Bioastronomy, IAU Coll. 161, Cosmovici C.B., Bowyer S. Werthimer D. eds., pp 89-96.

Charnley, S.B., Rodgers, S.D., Butner, H. M., Ehrenfreund, P. (2002a). Chemical Processes in Cometary Comae, Earth Moon and Planets, 90, 349.

Charnley, S.B., Rodgers, S.D., Kuan, Y.-J., Huang, H.-C. (2002b). Biomolecules in the interstellar medium and in comets, Advances in Space Research, 30, 1419.

Chen, J., Jewitt, D. (1994). On the rate at which comets split, Icarus, 108, 265.

Chyba, C., Sagan, C. (1997). Comets as a Source of Prebiotic Organic Molecules for the Early Earth, in Comets and the Origin and Evolution of Life, eds P.J. Thomas, C.F. Chyba, C.P. McKay, Springer.

Chyba, C.F., Thomas, P.J., Brookshaw, L., Sagan, C. (1990). Cometary delivery of organic molecules to the early earth. Science, 249, 249-373.

Combes F., Q-Rieu N., Wlodarczak, G. (1996). Search for interstellar glycine. Astronomy and Astrophysics, 308, 618-622.

Commeyras, A., Boiteau, L., Trambouze-Vandenabeele, O., Selsis, F. (2004). Lectures in Astrobiology - Vol. I, Eds. M. Gargaud, B. Barbier, H. Martin, J. Reisse, Springer Verlag, [this book] 
Cooper et al., (2001). Carbonaceous meteorites as a source of sugar-related organic compounds for the early Earth, Nature, 414, 879-883.

Coradini, A., Capaccioni, F., Capria, M. T., de Sanctis, M. C., Espinasse, S., Orosei, R., Salomone, M., Federico, C. (1997). Transition Elements between Comets and Asteroids, Icarus, 129, 337.

Cottin, H. (1999). Chimie organique de l'environnement cométaire: étude expérimentale de la contribution de la composante organique réfractaire à la phase gazeuse. Thèse, Univ. Paris 12.

Cottin, H., Gazeau, M.C., Raulin, F. (1999). Cometary organic chemistry: a review from observations, numerical and experimental simulations. Planet. Space Sci., 47, 1141-1162.

Cottin, H., Gazeau, M. C., Bénilan, Y., Raulin, F. (2001). Polyoxymethylene as parent molecule for the formaldehyde extended source in comet Halley. The Astrophysical Journal, 556, 417-420.

Cottin, H., Moore, M.H., Bénilan, Y. (2003). Photodestruction of relevant interstellar molecules in ice mixtures. The Astrophysical Journal, 590, 874-881.

Cottin, H., Szopa, C., Moore, M.H. (2001). Production of hexamethylenetetramine in photolyzed and irradiated interstellar cometary ice analogs. Astrophys. J. Lett., 561, L139-L142.

Cottin, H., Bachir, S., Raulin, F., Gazeau, M.C. (2002). Photodegradation of Hexamethylenetetramine by VUV and its relevance for $\mathrm{CN}$ and HCN extended sources in comets. Adv. Space Res., 30, 1481-1488.

Cottin, H., Bénilan, Y., Gazeau, M.-C., Raulin, F. (2004). Origin of cometary extended sources from degradation of refractory organics on grains: polyoxymethylene as formaldehyde parent molecule. Icarus, 167, 397-416.

Cremonese, G., Boehnhardt, H., Crovisier, J., Rauer, H., Fitzsimmons, A., Fulle, M., Licandro, J., Pollacco, D., Tozzi, G.P., West, R.M. (1997). Neutral Sodium from Comet Hale-Bopp: A Third Type of Tail. Astrophysical Journal Letters, 490, L199.

Crifo, J.F. (1988). Cometary dust sizing: comparison between optical and in-situ sampling techniques. Particle \&5 Particle Systems Characterization, 5, 38-46.

Crifo, J.F. (1991). Hydrodynamic models of the collisional coma. In Comets in the PostHalley Era (eds. R. L. Newburn, M. Neugebauer, J. Rahe), pp. 937-989. Kluwer.

Crifo, J.F. (1995). A general physicochemical model of the inner coma of active comets. I. Implications of spatially distributed gas and dust production. The Astrophysical Journal, 445, 470-488.

Crifo, J.F., Rodionov, A.V. (1997). The Dependence of the Circumnuclear Coma Structure on the Properties of the Nucleus. I. Comparison between a homogeneous and an Inhomogeneous Spherical Nucleus, with Application to P/Wirtanen. Icarus, 127, 319 .

Cronin, J., Chang, S. (1993). Organic matter in meteorites: Molecular and isotopic analysis. In The Chemistry of Life's origin, Greenberg et al., eds., Kluwer, 209-258.

Crovisier, J. (1999) Infrared Observations Of Volatile Molecules In Comet Hale-Bopp. Earth, Moon, and Planets, 79, 125-143.

Crovisier, J. (2004, in press) The molecular complexity of comets. Astrobiology: Future Perspectives, ISSI, Kluwer.

Crovisier, J., Bockelée-Morvan, D., Colom, P., Despois, D., Lis, D.C. et al., (submitted 2004a). The composition of ices in comet C/1995 O1 (Hale-Bopp) from radio spectroscopy: Further results and upper limits on undetected species. Astron. Astrophys. 
Crovisier, J., Bockelée-Morvan, D., Biver, N., Colom, P., Despois, D., Lis, D.C. (submitted 2004b). Ethylene glycol in comet C/1995 O1. Astron. Astrophys.

Crovisier, J. Schloerb, F.P. (1991) The Study of Comets at Radio Wavelengths. In Comets in the Post-Halley Era, Newburn R.L. Jr., Neugebauer M. Rahe J. eds., Kluwer, pp 149-174.

Crovisier, J., Encrenaz, T. (2000). Comet Science: The Study of Remnants From the Birth of the Solar System, Cambridge University Press

Crovisier, J., Leech, K., Bockelée-Morvan, D., Brooke, T.Y., Hanner, M. S., Altieri B., Keller H. U., Lellouch E. (1997). The Spectrum of Comet Hale-Bopp (C/1995 O1) Observed with the Infrared Space Observatory at 2.9 Astronomical Units from the Sun. Science, 275, 1904-1907.

Dauphas, N., Robert, F., Marty, B. (2000). The Late Asteroidal and Cometary Bombardment of Earth as Recorded in Water Deuterium to Protium Ratio, Icarus, 148, 508-512.

Dauphas, N., Marty, B. (2002). Inference on the nature and the mass of Earth's late veneer from noble metals and gases, Journal of Geophysical Research (Planets), 107, E12, 5129

De Bergh, C. (2004, in press). Kuiper Belt: water and organics. Astrobiology: Future Perspectives, ISSI, Kluwer.

Delsemme, A. H. (2000). 1999 Kuiper Prize Lecture Cometary Origin of the Biosphere. Icarus, 146, 313-325.

De Sanctis, M.C., Capria, M.T., Coradini, A. (2001). Thermal Evolution and Differentiation of Edgeworth-Kuiper Belt Objects, Astronomical Journal, 201, 2792.

Despois, D. (1999). Radio line observations of molecular and isotopic species in comet C/1995 O1 (Hale-Bopp). Earth Moon and Planets, 79, 103-124.

Despois, D., Ricaud, P., Lautié, N., Schneider, N., Jacq T., Biver, N., Lis, D.C., Chamberlin, R.A., Phillips, T.G., Miller, M., Jenniskens, P. (2000). Search for Extraterrestrial Origin of Atomic Trace Molecules - Radio Sub-mm Observations during the Leonids. Earth, Moon and Planets, 82-83, 129-140.

Despois, D. (1992). Solar System - Interstellar Medium a Chemical Memory of the Origins(rp), IAU Symp. 150: Astrochemistry of Cosmic Phenomena, 451.

Despois, D., Crovisier, J., Bockelée-Morvan, D., Biver, N. (2002). Comets and prebiotic chemistry: the volatile component, ESA SP-518: Exo-Astrobiology, 123.

Despois, D., Crovisier, J., Bockelee-Morvan, D., Colom, P. (1992). Formation of Comets: Constraints from the Abundance of Hydrogen Sulfide and Other Sulfur Species, IAU Symp. 150: Astrochemistry of Cosmic Phenomena, 459.

Duncan, M., Quinn, T., Tremaine, S. (1997). The formation and extent of the solar system comet cloud, Astronomical Journal, 94, 1330.

Eberhardt, P., Krankowsky, D. (1995). The electron temperature in the inner coma of comet P/Halley. Astron. Astophys., 295, 795.

Edgeworth, K.E. (1943). The evolution of our planetary system, Journal of the British Astronomical Association, 53, 181-186.

Ehrenfreund, P., Charnley, S.B. (2000). Organic molecules in the interstellar medium, comets and meteorites: a voyage from dark clouds to the early earth. Annual Review of Astronomy \& Astrophysics, 38, 427-483.

Ehrenfreund, P., Irvine, W., Becker, L., Blank, J., Brucato, J. R., Colangeli, L., Derenne, S., Despois, D., Dutrey, A., Fraaije, H., Lazcano, A., Owen, T., Robert, F. 
(2002). Astrophysical and astrochemical insights into the origin of life., Reports of Progress in Physics, 65, 1427.

Encrenaz, T., Knacke, R. (1991). Infrared Spectroscopy of Cometary Parent Molecules. In Comets in the Post-Halley Era, Newburn R.L. Jr., Neugebauer M. Rahe J. eds., Kluwer, 149-174.

Enzian, A., Klinger, J., Schwehm, G., Weissman, P. R. (1999). Temperature and Gas Production Distributions on the Surface of a Spherical Model Comet Nucleus in the Orbit of 46P/Wirtanen, Icarus, 138, 74.

Fassett, J.E., Lucas, S.G., Zielinski, R.A., Budahn, J.R. (2001). Compelling New Evidence for Paleocene Dinosaurs in the Ojo Alamo Sandstone San Juan Basin, New Mexico and Colorado, USA, Proceedings of the 32nd Symposium on Celestial Mechanics, 3139.

Fegley, B. Jr. (1993). Chemistry of the solar nebula, in The Chemistry of Life's Origin, eds J.M. Greenberg, C.X. Mendoza-Gomez, V. Pirronello, NATO ASI Series C, Kluwer, 416, 75-147.

Fernandez, J.A. (1980). On the existence of a comet belt beyond Neptune, Monthly Notices of the Royal Astronomical Society, 192, 481.

Fernandez, J.A. (1997). The Formation of the Oort Cloud and the Primitive Galactic Environment, Icarus, 129, 106.

Festou, M.C., Rickman, H.West R. M. (1993a). Comets. 1: Concepts and observations. Astronomy and Astrophysics Review, 4, 363-447.

Festou M.C., Rickman H.West R. M. (1993b). Comets. 2: Models, evolution, origin and outlook Astronomy and Astrophysics Review, 5, 37-163.

Festou, M., Keller, U., Weaver, H. (2004, in press) eds, Comets II, Univ. of Arizona Press.

Fomenkova, M.N. (1999). On the Organic Refractory Component of Cometary Dust. Space Science Reviews, 90, 109-114.

Frankel, Ch. (1999). The End of the Dinosaurs: Chicxulub Crater and Mass Extinctions Cambridge Univ. Press.

Fray, N., Bénilan, Y., Cottin, H., Gazeau, M.-C., Crovisier, J. (submitted). The origin of the CN radical in comets: A review from observations and models. Planetary and Space Science.

Gargaud, M., Despois, D., Parisot, J.-P. (2001). L'environnement de la Terre Primitive, Presses Universitaires de Bordeaux.

Gargaud, M., Despois, D., Parisot, J.-P., Reisse, J. (2003). Les Traces du Vivant, Presses Universitaires de Bordeaux.

Geiss, J. (1988). Composition in Halley's comet, Reviews in Modern Astron., 1, 1-27.

Geiss, J., Altwegg, K., Balsiger, H., Graf, S. (1999). Rare Atoms, Molecules and Radicals in the Coma of P/Halley. Space Science Reviews, 90, 253-268. Gerakines, P. A. M. H. Moore (2001). Carbon suboxide in astrophysical ice analogs. Icarus, 154, $372-380$.

Gerakines, P.A., Moore, M.H., Hudson, R.L. (2000). Carbonic acid production in $\mathrm{H}_{2} \mathrm{O}: \mathrm{CO}_{2}$ ices. UV photolysis vs. proton bombardment. Astron. Astophys., 357, 793-800.

Gibb, E.L., Mumma, M.J., dello Russo, N., Disanti, M.A., Magee-Sauer, K., (2003) Methane in Oort cloud comets, Icarus, 165, 391. 
Green, D.W.E. (1999). What is improper about the term "Kuiper belt"? (or, Why name a thing after a man who didn't believe its existence?) International Comet Quarterly, pp 44-46, January, 1999 and http://cfa-www.harvard.edu/icq/ICQpluto2.html.

Greenberg, J.M. (1982). What are comets made of ? A model based on interstellar dust. In Comets (ed. L. L. Wilkening), pp. 131-163. University of Arizona Press.

Greenberg, J.M., Li, A. (1997). Silicate core-organic refractory mantle particles as interstellar dust and as aggregated in comets and stellar disks. Adv. Space Res., 19, 981-990.

Greenberg, J.M., Li, A. (1998). From interstellar dust to comets: the extended CO source in comet Halley, Astron. Astophys., 332, 374-384.

Greenberg, J.M., Li, A., Mendoza-Gómez, C. X., Schutte, W. A., Gerakines, P. A., Groot, M. d. (1995). Approaching the Interstellar Grain Organic Refractory Component. Astrophys. J., 455, L177-L180.

Greenberg, J.M. (1998). Making a comet nucleus, Astronomy and Astrophysics, 330, 375 .

Greenberg, J.M. (1993). Physical and Chemical composition of Comets - From interstellar space to the Earth, in The Chemistry of Life's Origin, eds. J.M. Greenberg, C.X. Mendoza-Gomez, V. Pirronello, NATO ASI Series C, Kluwer, 416, pp195-208.

Greenberg, J. M. C. X. Mendoza-Gomez (1993). Interstellar dust evolution: a reservoir of prebiotic molecules. In The Chemistry of Life's Origins (ed. Greenberg), 1-32. Kluwer Academic.

Harker, D.E., Wooden, D.H., Woodward, C.E., Lisse, C.M. (2002). Grain Properties of Comet C/1995 O1 (Hale-Bopp). Astrophysical Journal, 580, 579-597.

Hayward, T.L. Hanner, M.S. (1997). Ground-Based Thermal Infrared Observations of Comet Hale-Bopp (C/1995 O1) During 1996. Science, 275, 1907-1909.

Hersant, F., Gautier, D., Huré, J.-M. (2001). A Two-dimensional Model for the Primordial Nebula Constrained by D/H Measurements in the Solar System: Implications for the Formation of Giant Planets. Astrophysical Journal, 554, 391-407.

Hollis, J.M., Lovas, F.J., Jewell, P.R. (2000). Interstellar Glycolaldehyde: The First Sugar. Astrophys. Journal Lett., 540, L107-L110.

Hollis, J.M., Pedelty, J.A., Snyder, L.E., Jewell, P.R., Lovas, F.J., Palmer, P., Liu, S.Y. (2003). A Sensitive Very Large Array Search for Small-Scale Glycine Emission toward OMC-1, Astrophysical Journal, 588, 353.

Hough, J.H., Bailey, J.A., Chrysostomou, A., Gledhill, T.M., Lucas, P. W., Tamura, M. Clark, S., Yates, J., Menard, F. (2001). Circular polarisation in star-forming regions: possible implications for homochirality, Advances in Space Research, 27, 313.

Hudson, R. L., Moore, M. H. (1999). Laboratory Studies of the Formation of Methanol and Other Organic Molecules by Water+Carbon Monoxide Radiolysis: Relevance to Comets, Icy Satellites, and Interstellar Ices. Icarus, 140, 451-461.

Hudson, R. L., Moore, M. H. (2002). The $\mathrm{N}_{3}$ Radical as a Discriminator between ion irradiated and UV-photolyzed astronomical ices. Astrophys. J., 568, 1095-1099.

Huebner, W. F. (1987). First polymer in space identified in comet Halley. Science, 237, $628-630$

Huebner, W. F., Boice D. C., Korth A. (1989). Halley's polymeric organic molecules. Advances in Space Research, 9, 29-34.

Huebner, W. F., Benkhoff, J. (1999a). From Coma Abundances to Nucleus Composition, Space Science Reviews, 90, 117. 
Huebner, W. F., Benkhoff, J., Capria, M. T., Coradini, A., de Sanctis, M. C., Enzian, A., Orosei, R., Prialnik, D. (1999b). Results from the Comet Nucleus Model Team at the International Space Science Institute, Bern, Switzerland, Advances in Space Research, 23, 1283.

Iro, N., Gautier, D., Hersant, F., Bockelée-Morvan, D., Lunine, J. I. (2003). An interpretation of the nitrogen deficiency in comets, Icarus, 161, 511.

Irvine, W. M., Schloerb F. P., Crovisier J., Fegley B., Mumma M. J. (2000a). Comets: a link between interstellar and nebular chemistry. In Protostar and Planets $I V$ (eds. V. Manning, A. Boss, S. Russel), pp. 1159. University of Arizona Press.

Irvine, W. M., Senay M., Lovell A. J., Matthews H. E., McGonagle D., Meier R. (2000b). Detection of Nitrogen Sulfide in Comet Hale-Bopp. Icarus, 143, 412-414.

Irvine, W., Bockelee-Morvan, D., Lis, D. C., Matthews, H. E., Biver, N., Crovisier, J., Davies, J. K., Dent, W. R. F., Gautier, D., Godfrey, P. D., Keene, J., Lowell, A. J., Owen, T. C., Phillips, T. G., Rauer, H., Schloerb, F. P., Senay, M., Young, K (1996). Spectroscopic evidence for interstellar ices in Comet Hyakutake, Nature, 382, 418.

Irvine, W. M., Bergman, P., Lowe, T. B., Matthews, H., McGonagle, D., Nummelin, A., Owen, T. (2003). HCN and HNC in Comets C/2000 Wm1 (Linear) and C/2002 C1 (Ikeya-Zhang), Origins of Life and Evolution of the Biosphere, 33, 609.

Irvine, W. M., Leschine, S. B., Schloerb, F. P. (1980). Thermal History, Chemical Composition and Relationship of Comets to the Origin of Life, Nature, 283, 748.

Jeffers, S. V., Manley, S. P., Bailey, M. E., Asher, D. J. (2001). Near-Earth object velocity distributions and consequences for the Chicxulub impactor, Monthly Notices of the Royal Astronomical Society, 327, 126.

Jenniskens, P. (2001). Meteors as a delivery vehicle for organic matter to the early Earth, in Meteoroids 2001 Conference, ESA SP-495, 247-254

Jenniskens, P., Baratta, G. A., Kouchi, A. Groot, M. S. D., Greenberg, J. M., Strazzulla, G. (1993). Carbon dust formation on interstellar grains. Astronomy and Astrophysics, 273, 583-600.

Jessberger, E. K., Kissel, J. (1991). Chemical properties of cometary dust and a note on carbon isotopes, ASSL Vol. 167: IAU Colloq. 116: Comets in the Post-Halley Era, 1075.

Jewitt, D. C., Matthews, H. E., Owen, T., Meier. R. (1997). Measurements of ${ }^{12} \mathrm{C} /{ }^{13} \mathrm{C}$, ${ }^{14} \mathrm{~N} /{ }^{15} \mathrm{~N}$, and ${ }^{32} \mathrm{~S} /{ }^{34} \mathrm{~S}$ Ratios in Comet Hale-Bopp (C/1995 O1). Science, 278, 9093.

Jewitt, D. (2002). From Kuiper Belt object to cometary nucleus, ESA SP-500: Asteroids, Comets, and Meteors, ACM 2002, 11.

Jewitt, D. C., Luu, J. X. (1993). Discovery of the Candidate Kuiper Belt Object 1992 QB1. Nature, 362, 730-732.

Jopek, T. J., Gonczi, R., Froeschle', C., Michel, P., Longo, G., Foschini, L. (2002). A main belt asteroid: the most probable cause of the Tunguska event, Memorie della Societa Astronomica Italiana, 73, 679.

Karmann, C., Beust, H., Klinger, J. (2003). The physico-chemical history of Falling Evaporating Bodies around beta Pictoris: The sublimation of refractory material, Astronomy and Astrophysics, 409, 347.

Karmann, C., Beust, H., Klinger, J. (2001). The physico-chemical history of Falling Evaporating Bodies around beta Pictoris: investigating the presence of volatiles, Astronomy and Astrophysics, 372, 616 . 
Kasamatsu, T., Kaneko, T., Saito, T., Kobayashi, K. (1997). Formation of organic compounds in simulated interstellar media with hich energy particles. Bulletin of the Chemical Society of Japan, 70, 1021-1026.

Kasting, J. F (1993). Earth's early atmosphere, Science, 259, 920-926.

Kasting, J. Catling, D. (2003). Evolution of a habitable planet, Ann. Rev. Astron. Astrophys, 41, 429-63.

Kissel, J. Krueger, F. R. (1987). The organic component in dust from comet Halley as mesured by the PUMA mass spectrometer on board Vega 1. Nature, 326, 755-760.

Kissel, J., Krueger, F.R. et Roessler, K. (1997). Organic Chemistry in Comets From Remote and In Situ Observations. In Comets and the Origins and Evolution of Life, Thomas P.J., Chyba C.P. McKay C.P. eds., Springer, 69-110.

Klavetter, J. J., A'Hearn, M. F. (1994). An extended source for CN jets in Comet $\mathrm{P} /$ Halley. Icarus, 107, 322-334.

Kobayashi, K., Kasamatsu, T., Kaneko, T., Koike, J., Oshima, T., Saito, T., Yamamoto, T., Yanagawa, H. (1995). Formation of amino acid precursors in cometary ice environments by cosmic radiation. Adv. Space Res., 16, (2)21-(2)26.

Krueger, F. R. Kissel, J. (1987). The chemical composition of the dust of comet $\mathrm{P} /$ Halley as measured by "Puma" on board Vega-1. Naturwissenschaften, 74, 312-316.

Krueger, F. R., Korth, A., Kissel, J. (1991). The organic matter of comet Halley as inferred by joint gas phase and solid phase analyses. Space Science Reviews, 56, $167-175$.

Kuan, Y., Charnley, S. B., Huang, H., Tseng, W., Kisiel, Z. (2003). Interstellar Glycine, Astrophysical Journal, 593, 848.

Kuiper, G.P. (1951). in Astrophysics: A Topical Symposium, ed. J. A. Hynek (New York: McGraw-Hill), 400.

Kurtz, S., Cesaroni, R., Churchwell, E., Hofner, P., Walmsley, C. M. (2000). Hot Molecular Cores and the Earliest Phases of High-Mass Star Formation, Protostars and Planets IV, 299.

Kyte, F.T. (1998). A meteorite from the Cretaceous/Tertiary boundary. Nature 396, $237-239$

Lagrange, A.-M., Backman, D. E., Artymowicz, P. (2000). Planetary Material around Main- Sequence Stars, Protostars and Planets IV, 639.

Lamy, P., Biesecker, D. (2002), The properties of Sun-grazing coments, $34^{\text {th }}$ COSPAR meeting, Houston, p 3159

Le Loeuff, J., Laurent, Y. (2001). Biodiversity of Late Maastrichtian Dinosaurs, Proceedings of the 32nd Symposium on Celestial Mechanics, 3126.

Lecacheux, A., Biver, N., Crovisier, J., Bockelée-Morvan, D., Baron, P., Booth, R. S., Encrenaz, P., Floren, H.-G., Frisk, U., Hjalmarson, A., Kwok, S., Mattila, K., Nordh, L., Olberg, M., Olofsson, A. O. H., Rickman, H., Sandqvist, A., von Scheele, F., Serra, G., Torchinsky, S., Volk, K., Winnberg, A. (2003). Observations of water in comets with Odin, Astronomy and Astrophysics, 402, L55.

Lecavelier Des Etangs, A., Vidal-Madjar, A., Ferlet, R. (1999). Photometric stellar variation due to extra-solar comets, Astronomy and Astrophysics, 343, 916.

Leonard (1930). Leaflet Astron. Soc. Pacific, 30, 21-124

Levasseur-Regourd, A. C., Hadamcik, E., Renard, J. B. (1996). Evidence for two classes of comets from their polarimetric properties at large phase angles. Astronomy and Astrophysics, 313, 327. 
Levison, H. F., Dones, L., Duncan, M. J. (2001). The Origin of Halley-Type Comets: Probing the Inner Oort Cloud. The Astronomical Journal, 121, 2253-2267.

Lewis, J. S., Prinn, R. G. (1980). Kinetic inhibition of $\mathrm{CO}$ and $\mathrm{N}_{2}$ reduction in the solar nebula, Astrophysical Journal, 238, 357.

Magee-Sauer, K. (2002). CSHELL Observations of Comet C/2002 C1(Ikeya-Zhang) in the 3.0-Micron Region, DPS Meeting, BAAS, 34, 16.05.

Malfait, K., Waelkens, C., Waters, L. B. F. M., Vandenbussche, B., Huygen, E., de Graauw, M. S. (1998). The spectrum of the young star HD 100546 observed with the Infrared Space Observatory. Astronomy and Astrophysics, 332, L25.

Maurette M. (1998). Carbonaceous Micrometeorites and the Origin of Life, Origins Life Evolution Biosphere, 28, 385-412.

Maurette, M., Duprat, J., Engrand, C., Gounelle, M., Kurat, G., Matrajt, G., Toppani, A. (2000). Accretion of neon, organics, $\mathrm{CO}_{2}$, nitrogen and water from large interplanetary dust particles on the early Earth. Planetary and Space Science, 48, 1117.

McDonnell, J. A. M., Lamy, P. L., Pankiewicz, G. S. (1991). Physical properties of cometary dust. In Comets in the Post-Halley Era, Vol. 2, pp. 1043-1073. Kluwer.

McKinnon, W. B. (2002). On the initial thermal evolution of Kuiper Belt objects, ESA SP-500: Asteroids, Comets, and Meteors: ACM 2002, 29.

Meier, R., Owen, T. C., Jewitt, D. C., Matthews, H. E., Senay, M., Biver, N., BockeleeMorvan, D., Crovisier, J., Gautier, D. (1998). Deuterium in Comet C/1995 O1 (HaleBopp): Detection of DCN, Science, 279, 1707.

Miller S.L. (1998). The endogenous synthesis of organic compounds, in The Molecular Origin of Life: Assembling Pieces of the Puzzle, A. Brack ed., Cambridge, 59-85.

Mitchell, D. L., Lin, R. P., Carlson, C. W., Korth, A., Rème, H., Mendis, D. A. (1992). The origin of complex organic ions in the coma of comet Halley. Icarus, 98, 125-133. Montmerle chapter this book.

Moore, M. H., R. Khanna, B. Donn (1991). Studies of Proton Irradiated $\mathrm{H}_{2} \mathrm{O}+\mathrm{CO}_{2}$ and $\mathrm{H}_{2} \mathrm{O}+\mathrm{CO}$ Ices and Analysis of Synthesized Molecules. J. Geophys. Res., 96, $17541-17545$.

Morbidelli, A. Brown, M.E. (2004, in press). The Kuiper belt and the primordial evolution of the Solar System in Comets II, eds. M. Festou, U. Keller, H. Weaver, Univ. of Arizona Press.

Morbidelli, A., Chambers, J., Lunine, J. I., Petit, J. M., Robert, F., Valsecchi, G. B., Cyr, K. E. (2000). Source regions and time scales for the delivery of water to Earth, Meteoritics and Planetary Science, 35, 1309.

Moreels, G., Clairemidi, J., Hermine, P., Brechignac, P., Rousselot, P. (1994). Detection of a polycyclic aromatic molecule in comet $\mathrm{P} / \mathrm{Halley}$. Astronomy and Astrophysics, 282, 643-656.

Moreno, R. (1998). Observations millimétriques et submillimétriques des planètes géantes. Etude de Jupiter après la chute de la comète SL9. PhD Thesis, Université de Paris VI.

Mousis, O., Gautier, D., Bockelée-Morvan, D., Robert, F., Dubrulle, B., Drouart, A. (2000). Constraints on the Formation of Comets from D/H Ratios Measured in $\mathrm{H}_{2} \mathrm{O}$ and HCN, Icarus, , 148, 513.

Mumma, M. J., Weissman, P. R., Stern, S. A. (1993). Comets and the Origin of the solar system: reading the Rosetta stone. In Protostars and Planets III, eds. E.H. Levy J. I. Lunine, Univ. of Arizona Press, pp 1177-1252. 
Mumma, M. J., DiSanti, M. A., Dello Russo N., Magee-Sauer, K., Gibb, E., Novak, R. (2002), The organic volatile composition of Oort cloud comets: evidence for chemical diversity in the Giant-Planets nebular region., in Asteroids Comets Meteors 2002, ESA SP-500, 753.

Muñoz Caro, G. M. (2003). From photoprocessing of interstellar ice to amino acids and other organics. PhD Thesis, Leiden Observatory.

Muñoz Caro, G. M., Meierhenrich, U. J., Schutte, W. A., Barbier, B., Arcones Segovia, A., Rosenbauer, H., Thiemann, W. H.-P., Brack, A., Greenberg, J. M. (2002). Amino acids from ultraviolet irradiation of interstellar ice analogues. Nature, 416, 403-406.

Navarro-Gonzalez, R. A. Romero (1996). On the Survivability of an Enantiomeric Excess of Amino Acids in Comet Nuclei During the Decay of 26 A1 and Other radionuclides. Astrophys. Space Sci., 236, 49-60.

Navarro-Gonzalez, R., Ponnamperuma, C., Khanna, R. K. (1992). Computational study of radiation chemical processing in comet nuclei. Orig. Life, 21, 359-374.

Newburn, R. L.,Jr., Neugebauer, M. Rahe J. eds (1991). Comets in the Post-Halley Era, Kluwer

Noll, K.S., Weaver, H.A., Feldman, P.D. eds. (1996). The Collision of Comet ShoemakerLevy 9 and Jupiter, Cambridge University Press.

Notesco, G., Bar-Nun, A. (1996). Enrichment of CO over $\mathrm{N}_{2}$ by their Trapping in Amorphous Ice and Implications to Comet P/Halley. Icarus, 122, 118-121.

Notesco, G., Bar-Nun, A. (1997). Trapping of Methanol, Hydrogen Cyanide, and nHexane in Water Ice, above Its Transformation Temperature to the Crystalline Form. Icarus, 126, 336-341.

Notesco, G., Laufer, D., Bar-Nun, A. (1997). The Source of the High $\mathrm{C}_{2} \mathrm{H}_{6} / \mathrm{CH}_{4}$ Ratio in Comet Hyakutake. Icarus, 125, 471-473.

Nuth, J. A., III, Rietmeijer, F. J. M., Hill, H. G. M. (2002). Condensation processes in astrophysical environments: The composition and structure of cometary grains. Meteoritics and Planetary Science, 37, 1579-1590.

Oort, J. H. (1950). The structure of the cloud of comets surrounding the Solar System and a hypothesis concerning its origin, Bulletin of the Astronomical Institute of the Netherlands, 11, 91.

Oró, J. (1961). Comets and the formation of biochemical compounds on the primitive Earth. Nature, 190, 389-390.

Oró, J. (2001). Cometary molecules and Life's origin, in First Steps in the Origin of Life in the Universe, Chela-Flores et al., eds., Kluwer, 113-120.

Oró, J. C. B. Cosmovici (1997). Comets and Life on the primitive Earth. In Astronomical and Biochemical Origins and the Search for Life in the Universe (eds. C. B. Cosmovici, S. Bowyer, D. Werthimer), 97-120.

Pendleton, Y. J., Sandford, S. A., Allamandola, L. J., Tielens, A. G. G., Sellgren, K. (1994). Near-Infrared Absorption spectroscopy of interstellar hydrocarbon grains. Astrophys. J., 437, 683-696.

Petit, J.-M., Morbidelli, A. (2004). Lectures in Astrobiology - Vol. I, eds. M. Gargaud, B. Barbier, H. Martin, J. Reisse, Springer Verlag, [this book].

Podolak, M., Prialnik, D. (1997). ${ }^{26}$ Al and Liquid Water Environment in Comets in Comets and the Origin and Evolution of Life, Springer.

Prialnik, D. (2002). Modeling the Comet Nucleus Interior, Earth Moon and Planets, 89, 27. 
Prialnik, D. (1999). Modelling Gas and Dust Release from Comet Hale-Bopp, Earth Moon and Planets, , 77, 223.

Prialnik, D., Podolak, M. (1999). Changes in the Structure of Comet Nuclei Due to Radioactive Heating, Space Science Reviews, 90, 169.

Prinn, R. G., Fegley, B. (1981). Kinetic inhibition of CO and $\mathrm{N}_{2}$ reduction in circumplanetary nebulae - Implications for satellite composition, Astrophysical Journal, 249, 308.

Prinn, R. G. Fegley, B. Jr. (1989). Solar Nebula Chemistry: Origin of planetary, satellite and cometary volatiles. In Origin and Evolution of Planetary and Satellite Atmospheres, eds S.K. Atreya, J. B. Pollack, M. Matthews (1989) Univ. Of Arizona Press, pp 78-136.

Radicati-Di-Brozolo, F., Bunch, T. E., Chang, S. (1986). Laser microprobe study of carbon in interplanetary dust particles. Orig. Life, 16, 236-237.

Rairden, R. L., Jenniskens, P., Laux, C. O. (2000). Search for Organic Matter in Leonid Meteoroids, Earth Moon and Planets, 82, 71.

Robert, F. (2002). Water and organic matter D/H ratios in the solar system: a record of an early irradiation of the nebula? Planetary and Space Science, 50, 1227-1234.

Rocchia, R. et Robin E. (2000) L'origine extraterrestre de la crise Crétacé-Tertiaire. Pour la Science (juillet 2000) La Valse des Espèces. Dossier Hors-Série, Belin, pp 100-111.

Rodgers, S. D., Charnley, S. B. (1998). HNC and HCN in Comets. The Astrophysical Journal Letters, 501, L227-L230.

Rodgers, S. D., Charnley, S. B. (2001a). Organic synthesis in the coma of Comet HaleBopp? Monthly Notices of the Royal Astronomical Society, 320, L61-64.

Rodgers, S. D., Charnley, S. B. (2001b). On the origin of HNC in Comet Lee, Monthly Notices of the Royal Astronomical Society, 323, 84-92.

Rolfe, E., Battrick, B. eds. (1987). Proceedings of the International Symposium on the Diversity and Similarity of Comets, ESA SP-278

Rosenbauer, H., Fuselier, S. A., Ghielmetti, A., Greenberg, J. M., Goesmann, F., Ulamec, S., Israel, G., Livi, S., MacDermott, J. A., Matsuo, T., Pillinger, C. T., Raulin, F., Roll, R., Thiemann, W. (1999). The COSAC experiment on the lander of the ROSETTA mission, Advances in Space Research, 23, 333.

Sankey, J. T. (2001). Late Cretaceous Theropod Dinosaur Diversity: Latitudinal Differences in North America and Implications for the K/T Extinctions, Proceedings of the $32^{\text {nd }}$ Symposium on Celestial Mechanics, 3148.

Shimoyama, A., Ogasawara, R. (2002). Dipeptides and Diketopiperazines in the Yamato791198 and Murchison Carbonaceous Chondrites. Origins of Life and the Biosphere, 32, 165 .

Schutte, W. A., L. J. Allamandola, S. A. Sandford (1993a). Formaldehyde and organic molecule production in astrophysical ices at cryogenic temperatures. Science, 259, $1143-1145$.

Schutte, W. A., L. J. Allamandola, S. A. Sandford (1993b). An Experimental Study of the Organic Molecules Produced in Cometary and Interstellar Ice Analogs by Thermal Formaldehyde Reactions. Icarus, 104, 118-137.

Shu, F. H., Shang, H., Lee, T. (1996). Toward an Astrophysical Theory of Chondrites, Science, 271, 1545.

Shuvalov, V. V., Artemieva, N. A. (2002). Numerical modeling of Tunguska-like impacts, Planetary and Space Science, 50, 181. 
Snyder, L. E. (1997). The Search for Interstellar Glycine. Origins of Life and Evolution of the Biosphere, 27, 115-133.

Steel, D. (1997). Cometary Impacts on the Biosphere. In Comets and the Origins and Evolution of Life, Thomas P.J., Chyba C.P. McKay C.P. eds, Springer, 209-242.

Stern, S. Alan (2003). The evolution of comets in the Oort cloud and Kuiper belt. Nature, 424, 639.

Strazzulla, G. (1997). Ion irradiation: its relevance to the evolution of complex organics in the outer solar system. Adv. Space Res., 19, 1077-1084.

Strazzulla, G., Baratta, G. A. (1991). Laboratory study of the IR spectrum of ionirradiated frozen benzene. Astronomy and Astrophysics, 241, 310-316.

Strazzulla, G., Baratta, G. A., Johnson, R. E., Donn, B. (1991). Primordial comet mantle: irradiation production of a stable, organic crust. Icarus, 91, 101-104.

Szopa, C., Sternberg, R., Raulin, F., Rosenbauer, H. (2003). What can we expect from the in situ chemical investigation of a cometary nucleus by gas chromatography: First results from laboratory studies. Planetary and Space Science, 51, 863-877.

Thomas, P.J., Chyba, C.P., McKay, C.P. eds (1997). Comets and the Origins and Evolution of Life, Springer

Waelkens, C., Malfait K. Waters L.B.F.M. (1999) Comet Hale-Bopp, Circumstellar Dust, and the Interstellar Medium. Earth, Moon and Planets, 79, 265-274.

Wakamutsu, H., Y. Yamada, T. Saito, I. Kumashiro, T. Takenishi (1966). Synthesis of Adenine by Oligomerization of Hydrogen Cyanide. Journal of Organic Chemistry, 31, 2035-2036.

Walker, J. F. (1964). Formaldehyde. Reinhold.

Weaver, H. A., Lamy, P. L. (1999). Estimating the Size of Hale-Bopp's Nucleus, Earth Moon and Planets, 79, 17.

Weidenschilling, S. J. (1997). The Origin of Comets in the Solar Nebula: A Unified Model, Icarus, 127, 290.

Weiler, M., Rauer, H., Helbert, J. (2004). Optical observations of Comet 67P/ ChuryumovGerasimenko (2004) Astronomy and Astrophysics, 414, 749.

Whipple, F. L. (1950). A comet model. I. The acceleration of Comet Encke. Astrophys. J., 111, 375-394.

Whittet, D.C.B. ed., (1997). Planetary and Interstellar Processes Relevant to the Origin of Life, Kluwer.

Whittet, D. C. B. (1997). Is Extraterrestrial Organic Matter Relevant to the Origin of Life on Earth?, Origins of Life and Evolution of the Biosphere, 27, 249.

Wolman, Y., S. L. Miller, J. Ibanez, J. Oro (1971). Science, 174, 1039.

Wooden, D. H., Butner, H. M., Harker, D. E., Woodward, C. E. (2000). Mg-Rich Silicate Crystals in Comet Hale-Bopp: ISM Relics or Solar Nebula Condensates? Icarus, 143, $126-137$.

Wootten, H. A. (2003). http://www.cv.nrao.edu/awootten/allmols.html.

Yamamoto, T. (1991). Chemical theories on the origin of comets, ASSL Vol. 167: IAU Colloq. 116: Comets in the Post-Halley Era, 361.

Yamamoto, T. (1985). Formation environment of cometary nuclei in the primordial solar nebula, Astronomy and Astrophysics, 142, 31-36.

Yamamoto, T., Nakagawa, N., Fukui, Y. (1983). The chemical composition and thermal history of the ice of a cometary nucleus, Astronomy and Astrophysics, 122, 171.

Zahnle, K. (1998). Origins of Atmospheres, ASP Conf. Ser. 148: Origins, 364-391. 